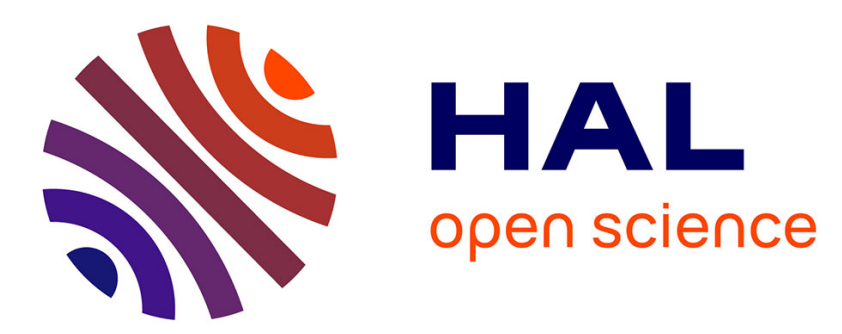

\title{
Nitsche-XFEM for the coupling of an incompressible fluid with immersed thin-walled structures
}

Frédéric Alauzet, Benoit Fabrèges, Miguel Angel Fernández, Mikel Landajuela

\section{To cite this version:}

Frédéric Alauzet, Benoit Fabrèges, Miguel Angel Fernández, Mikel Landajuela. Nitsche-XFEM for the coupling of an incompressible fluid with immersed thin-walled structures. Computer Methods in Applied Mechanics and Engineering, 2016, 301, pp.300-335. 10.1016/j.cma.2015.12.015 . hal$01149225 \mathrm{v} 3$

\section{HAL Id: hal-01149225 \\ https://hal.inria.fr/hal-01149225v3}

Submitted on 17 Dec 2015

HAL is a multi-disciplinary open access archive for the deposit and dissemination of scientific research documents, whether they are published or not. The documents may come from teaching and research institutions in France or abroad, or from public or private research centers.
L'archive ouverte pluridisciplinaire HAL, est destinée au dépôt et à la diffusion de documents scientifiques de niveau recherche, publiés ou non, émanant des établissements d'enseignement et de recherche français ou étrangers, des laboratoires publics ou privés. 
Nitsche-XFEM for the coupling of an incompressible fluid with immersed thin-walled

\section{structures}

Frédéric Alauzet, Benoit Fabrèges, Miguel A. Fernández, Mikel Landajuela

RESEARCH

REPORT

$\mathbf{N}^{\circ} 8723$

May 2015

Project-Teams GAMMA3 \& REO 



\title{
Giva

\section{Nitsche-XFEM for the coupling of an incompressible fluid with immersed thin-walled structures}

\author{
Frédéric Alauzet* Benoit Fabrèges \\ Mikel Landajuela ${ }^{\dagger \ddagger}$ \\ Project-Teams GAMMA3 \& REO
}

Research Report n 8723 - May 2015 - 48 pages

\begin{abstract}
In this paper we introduce a Nitsche-XFEM method for fluid-structure interaction problems involving a thin-walled elastic structure (Lagrangian formalism) immersed in an incompressible viscous fluid (Eulerian formalism). The fluid domain is discretized with an unstructured mesh not fitted to the solid mid-surface mesh. Weak and strong discontinuities across the interface are allowed for the velocity and pressure, respectively. The fluid-solid coupling is enforced consistently using a variant of Nitsche's method with cut-elements. Robustness with respect to arbitrary interface intersections is guaranteed through suitable stabilization. Several coupling schemes with different degrees of fluid-solid time splitting (implicit, semi-implicit and explicit) are investigated. A series of numerical test in 2D, involving static and moving interfaces, illustrates the performance of the different methods proposed.
\end{abstract}

Key-words: fluid-structure interaction, incompressible fluid, thin-walled structure, unfitted meshes, fictitious domain method, XFEM, Nitsche's method, cupling schemes.

* Inria, GAMMA3 project-team, Rocquencourt - B.P. 105, F-78153 Le Chesnay cedex, France

$\dagger$ Inria, REO project-team, Rocquencourt - B.P. 105, F-78153 Le Chesnay cedex, France

$¥$ Sorbonne Universités, UPMC Univ. Paris 6, Lab. Jacques-Louis Lions, 4 Place Jussieu, 75252 Paris cedex 05, France

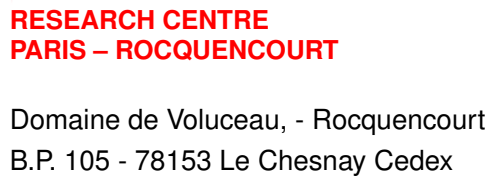




\section{Nitsche-XFEM pour le couplage d'un fluide incompressible avec des structures minces immergées}

Résumé : Dans cet article, nous présentons une méthode de type Nitsche-XFEM pour des problèmes d'interaction fluide-structure comportant une structure élastique mince (formalisme lagrangien) immergé dans un fluide visqueux incompressible (formalisme eulérien). Le domaine fluide est discrétisé avec un maillage non structuré incompatible avec celui du solide. La vitesse et la pression discrètes permettent des discontinuités faibles et fortes à travers l'interface, respectivement. Le couplage fluide-solide est traité de manière consistante en utilisant une variante de la méthode de Nitsche avec éléments coupés. La méthode est robuste par rapport à des intersections arbitraires entre les maillages fluide et solide grâce à des termes de stabilisation appropriés. Plusieurs types de schémas de couplage (implicite, semi-implicite et explicite) sont étudiés. Une série de tests numérique en $2 \mathrm{D}$, avec des interfaces statiques et mobiles, illustre la performance des différentes méthodes proposées.

Mots-clés : interaction fluide-structure, fluide incompressible, structure mince, maillages non compatibles, méthode de domaine fictif, XFEM, méthode de Nitsche, schémas de couplage. 


\section{Introduction}

Mathematical models describing the mechanical interaction of an incompressible viscous fluid with an immersed thin-walled flexible structure appear in a wide variety of engineering fields: from micro-encapsulation to the aeroelasticity of parachutes and sailing boats (see, e.g., [59, 67, 51]). Such multi-physics systems are also particularly ubiquitous in nature. One can think, for instance, of the wings of a bird interacting with the air, the fins of a fish moving through the water, or the opening/closing dynamics of heart valves when blood is propelled into the arteries (see, e.g., [70, 55, 44). The solid is deformed under the action of the fluid and the fluid flow is disturbed by the moving solid.

These problems are generally modeled by heterogeneous (parabolic/hyperbolic) systems of equations with different types of constitutive and geometrical non-linearities. This complicates the analysis both from the mathematical and numerical standpoint. In addition, the thin-walled nature of the immersed solid introduces jumps on the fluid stresses which, respectively, results in weak and strong discontinuities of the velocity and pressure fields. Standard finite element approximations, not allowing for such discontinuities, are known to deliver suboptimal convergence behavior and spurious numerical oscillations in the vicinity of the immersed solid (see, e.g., [27, 47, 8])

The discontinuous features of the fluid solution can be straightforwardly incorporated within a standard finite element approximation by considering fitted fluid-solid meshes. It is well known, however, that maintaining fitted meshes may be cumbersome or unfeasible in presence of large interface deflections and topological changes (e.g., due to contacting solids). Though a number of advanced mesh update techniques have been reported in the literature (see, e.g., 64, 73, 72, 1, 68]), the favoured alternative is to consider an unfitted mesh formulation, in which the fluidstructure interface moves independently of a background fluid mesh. Among these approaches, we can mention the Immersed Boundary/Fictitious Domain methods (e.g., [58, 40, 5, 74, 27, 4, 7, 47, 8]) and the methodologies based on a fully Eulerian description of the problem (e.g., 24, 60 ). In general, these methods are known to be inaccurate in space due to the continuous nature of the fluid approximations across the interface or to the discrete treatment of the interface conditions. The current trend to overcome these consistency issues is to combine a local XFEM enrichment with a cut-FEM methodology and a Lagrange multiplier treatment of the interface coupling (see, e.g., 48, 75, 39, 62 ). The price to pay, with respect to the original IB and FD methods, is the need of a specific tracking of the interface intersections (see, e.g., [54, 71, 53]) and a loss of robustness with respect to how the interface intersects the background fluid mesh (see, e.g., 34, 13]).

A well-known alternative to the discrete treatment of the interface conditions via Lagrange multipliers is Nitsche's method (see, e.g., [57, 65, 43]). Because of its flexibility and mathematical soundness, the Nitsche mortaring has been applied to the design of numerical methods for a number of interface problems, including XFEM for elasticity [41, 42, 6], XFEM for two-phase transport problems [49, 50], XFEM for incompressible flow 63] and robust and accurate FD methods for elliptic and mixed problems [20, [52, 21]. Nitsche's method was first applied to fluid-structure interaction problems with fitted meshes in [44 and used to design stable explicit coupling (or loosely coupled) schemes in [15, 16. It has recently been extended to fluid-structure interaction problems with unfitted meshes in [18, yielding robust and optimal a priori error estimates (fixed interface). In [18, the case of the coupling with thin-walled solids is limited to structures surrounding the fluid domain (i.e., not immersed).

The first contribution of this paper consists in the introduction of a robust and accurate Nitsche-XFEM method for fluid-structure interaction problems involving a thin-walled elastic structure immersed in an incompressible viscous fluid. We consider an Eulerian description for 
the fluid and a Lagrangian formulation for the solid. The fluid domain is discretized with an unstructured mesh not fitted to the solid mid-surface deformed mesh. In this unfitted mesh framework, the (strong) consistency of the proposed fluid-solid coupling builds on the following two ingredients:

- across the interface, locally enriched piecewise affine fluid velocity and pressure approximations respectively allow for weak and strong discontinuities (using the XFEM approach of [42, 6]);

- the kinematic/dynamic fluid-solid coupling is enforced through a fluid-sided Nitsche's mortaring (based on [18]).

Besides, consistent symmetric stabilization operators are added to guarantee robustness with respect to arbitrary interface/element intersections (see, e.g., 21]) and to circumvent the classical inf-sup and convective related instabilities (see, e.g., [10, 19, 14, 63]). In this regard, it is worth noting that for robustness these operators act on the fictitious region of the computational domain, without compromising the overall optimal accuracy of the method (in the energy norm).

The second contribution has to do with the time-discretization. Several coupling schemes with different levels of fluid-solid splitting are proposed: implicit, explicit and semi-implicit. The stability and convergence properties of the resulting fully discrete methods are analyzed within a representative linear setting (static interfaces). The salient features of the semi-implicit schemes introduced in this paper are twofold: (i) they deliver superior stability and accuracy with respect to alternative methods of explicit nature (see, e.g., [7]); (ii) they avoid the strong coupling of alternative semi-implicit coupling schemes (see, e.g., [56, 8]) without compromising stability and accuracy.

Finally, the theoretical findings are substantiated by a series of numerical examples in 2D, involving static and moving interfaces, which illustrate the performance of the methods proposed by comparing with analytic solutions and fitted mesh approaches.

The rest of the paper is organized as follows. Section 2 is devoted to the derivation and the analysis of the methods within a linear setting (fixed interface). The space semi-discrete NitscheXFEM formulation is introduced in Section 2.1. Section 2.2 presents the time discretization and the different coupling schemes. In Section 3, the numerical methods are formulated within a non-linear setting involving moving interfaces. Numerical evidence illustrating the performance of the methods proposed is reported and discussed in Section 4 . Finally, a summary of the conclusions and some directions of further investigation are given in Section 5.

\section{Linear model problem: static interface}

We consider a fluid-structure interaction problem in which the fluid is described by the Stokes equations and the structure by a linear thin membrane or shell model. The fluid domain is denoted by $\Omega \subset \mathbb{R}^{d}(d=2,3)$. The structure is assumed to be immersed within $\Omega$, with its mid-surface represented by the oriented manifold $\Sigma \subset \Omega$ of codimension 1 and unitary normal vector $\boldsymbol{n}$. For the time being, we assume that $\Sigma$ divides $\Omega$ into two open domains $\Omega_{1}$ and $\Omega_{2}$ (see Figure 1). In Section 2.1.3 we address the general case in which $\Omega$ is partially intersected by $\Sigma$. We denote the outward unit normal to $\Omega_{i}$ on $\Sigma$ by $\boldsymbol{n}_{i}, i=1,2$. Note that we choose $\Omega_{1}$ and $\Omega_{2}$ so that $\boldsymbol{n}_{1}=\boldsymbol{n}$ and $\boldsymbol{n}_{2}=-\boldsymbol{n}$. We set $\Gamma_{i}=\partial \Omega_{i} \backslash \Sigma, i=1,2$.

In the following we will make extensive use of the following definition. For a given continuous scalar or tensorial field $f$ defined in $\Omega$ (possibly discontinuous across $\Sigma$ ) we define its sided- 


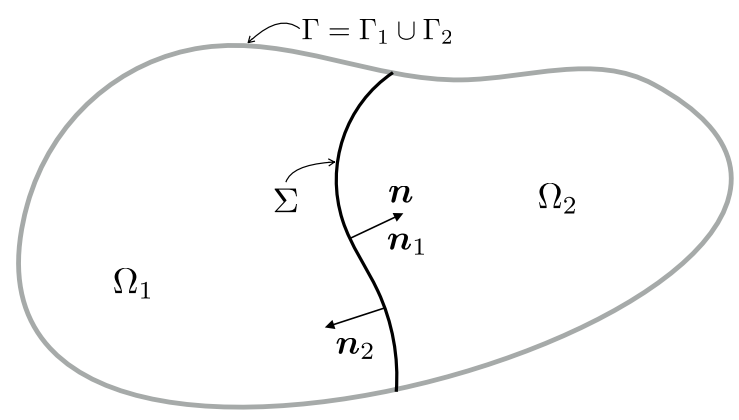

Figure 1: Geometric description.

restrictions to $\Sigma$, noted by $f_{1}$ and $f_{2}$, as

$$
f_{1}(\boldsymbol{x}) \stackrel{\text { def }}{=} \lim _{\xi \rightarrow 0^{+}} f\left(\boldsymbol{x}+\xi \boldsymbol{n}_{1}\right), \quad f_{2}(\boldsymbol{x}) \stackrel{\text { def }}{=} \lim _{\xi \rightarrow 0^{+}} f\left(\boldsymbol{x}+\xi \boldsymbol{n}_{2}\right) \quad \forall \boldsymbol{x} \in \Sigma .
$$

We also define the following jumps and average across the interface $\Sigma$ :

$$
\llbracket f \rrbracket \stackrel{\text { def }}{=} f_{1}-f_{2}, \quad \llbracket f \boldsymbol{n} \rrbracket \stackrel{\text { def }}{=} f_{1} \boldsymbol{n}_{1}+f_{2} \boldsymbol{n}_{2}, \quad\{f\} \stackrel{\text { def }}{=} \frac{1}{2}\left(f_{1}+f_{2}\right) .
$$

The coupled problem reads as follows: find the fluid velocity and pressure $\boldsymbol{u}: \Omega \times \mathbb{R}^{+} \rightarrow \mathbb{R}^{d}$, $p: \Omega \times \mathbb{R}^{+} \rightarrow \mathbb{R}$, the solid displacement and velocity $\boldsymbol{d}: \Sigma \times \mathbb{R}^{+} \rightarrow \mathbb{R}^{d}, \dot{\boldsymbol{d}}: \Sigma \times \mathbb{R}^{+} \rightarrow \mathbb{R}^{d}$ such that

$$
\begin{aligned}
& \left\{\begin{array}{rlll}
\rho^{\mathrm{f}} \partial_{t} \boldsymbol{u}-\operatorname{div} \boldsymbol{\sigma}(\boldsymbol{u}, p)=\mathbf{0} & \text { in } & \Omega_{i} \times \mathbb{R}^{+}, & i=1,2 \\
\operatorname{div} \boldsymbol{u}=0 & \text { in } & \Omega_{i} \times \mathbb{R}^{+}, & i=1,2 \\
\boldsymbol{u}=\mathbf{0} & \text { on } & \Gamma_{i} \times \mathbb{R}^{+}, & i=1,2
\end{array}\right. \\
& \left\{\begin{array}{rlc}
\boldsymbol{u}_{1}=\boldsymbol{u}_{2}=\dot{\boldsymbol{d}} & \text { on } & \Sigma \times \mathbb{R}^{+}, \\
\rho^{\mathrm{s}} \epsilon \partial_{t} \dot{\boldsymbol{d}}+\boldsymbol{L d}=-\llbracket \boldsymbol{\sigma}(\boldsymbol{u}, p) \boldsymbol{n} \rrbracket & \text { in } & \Sigma \times \mathbb{R}^{+}, \\
\dot{\boldsymbol{d}}=\partial_{t} \boldsymbol{d} & \text { in } & \Sigma \times \mathbb{R}^{+}, \\
\boldsymbol{d}=\mathbf{0} & \text { on } & \partial \Sigma \times \mathbb{R}^{+},
\end{array}\right.
\end{aligned}
$$

complemented with standard initial conditions $\boldsymbol{u}(0)=\boldsymbol{u}_{0}, \boldsymbol{d}(0)=\boldsymbol{d}_{0}$ and $\dot{\boldsymbol{d}}(0)=\dot{\boldsymbol{d}}_{0}$. Here, the constants $\rho^{\mathrm{f}}$ and $\rho^{\mathrm{s}}$ stand for the fluid and solid densities, respectively, while $\epsilon$ denotes the solid thickness. The fluid Cauchy-stress tensor is given by $\boldsymbol{\sigma}(\boldsymbol{u}, p) \stackrel{\text { def }}{=}-p \boldsymbol{I}+2 \mu \boldsymbol{\epsilon}(\boldsymbol{u})$ with $\boldsymbol{\epsilon}(\boldsymbol{u}) \stackrel{\text { def }}{=}$ $\frac{1}{2}\left(\boldsymbol{\nabla} \boldsymbol{u}+\boldsymbol{\nabla} \boldsymbol{u}^{\mathrm{T}}\right)$ and $\mu$ denoting the fluid dynamic viscosity. The abstract surface differential operator $\boldsymbol{L}$ describes the solid elastic effects.

Remark 2.1. Since in (1)-(2) the solid is geometrically modeled as a surface of co-dimension one, it does not displace any fluid. In presence of gravitational forcing, the relation $22_{1}$ should hence be replaced by

$$
\rho^{\mathrm{s}} \epsilon \partial_{t} \dot{\boldsymbol{d}}+\boldsymbol{L} \boldsymbol{d}=-\llbracket \boldsymbol{\sigma}(\boldsymbol{u}, p) \boldsymbol{n} \rrbracket+\left(\rho^{\mathrm{s}}-\rho^{\mathrm{f}}\right) \epsilon \boldsymbol{g} \quad \text { in } \quad \Sigma \times \mathbb{R}^{+},
$$

where $\boldsymbol{g}$ denotes the gravitational acceleration field and the term $-\rho^{\mathrm{f}} \boldsymbol{\epsilon} \boldsymbol{g}$ models the buoyant force acting on the solid (i.e, the displaced fluid).

$\mathrm{RR} \mathrm{n}^{\circ} 8723$ 
We will make use of the standard Sobolev spaces $H^{m}(\omega)(m \geq 0)$, with norm $\|\cdot\|_{m, \omega}$. The closed subspaces $H_{\Gamma}^{1}(\omega)$, of functions in $H^{1}(\omega)$ with zero trace on $\Gamma$, and $L_{0}^{2}(\omega)$, of functions in $L^{2}(\omega)$ with zero mean in $\omega$, will also be used. The scalar product in $L^{2}(\omega)$ is denoted by $(\cdot, \cdot)_{\omega}$ and its norm by $\|\cdot\|_{0, \omega}$. We consider $\boldsymbol{V} \stackrel{\text { def }}{=}\left[H_{\Gamma}^{1}(\Omega)\right]^{d}$ and $Q \stackrel{\text { def }}{=} L_{0}^{2}(\Omega)$ as the fluid velocity and pressure functional spaces, respectively. The space $\boldsymbol{W} \subset\left[H_{0}^{1}(\Sigma)\right]^{d}$ denotes the space of solid admissible displacements.

The standard Stokes bi-linear form

$$
a_{\Omega}^{\mathrm{f}}((\boldsymbol{u}, p),(\boldsymbol{v}, q)) \stackrel{\text { def }}{=} 2 \mu(\boldsymbol{\epsilon}(\boldsymbol{u}), \boldsymbol{\epsilon}(\boldsymbol{v}))_{\Omega}-(p, \operatorname{div} \boldsymbol{v})_{\Omega}+(q, \operatorname{div} \boldsymbol{u})_{\Omega}
$$

will be used. The elastic bi-linear form $a^{\mathrm{s}}: \boldsymbol{W} \times \boldsymbol{W} \rightarrow \mathbb{R}$ will represent the weak form of the (unbounded linear) surface differential operator $\boldsymbol{L}: \boldsymbol{D}(\boldsymbol{L}) \subset\left[L^{2}(\Sigma)\right]^{d} \rightarrow\left[L^{2}(\Sigma)\right]^{d}$, namely,

$$
a^{\mathrm{s}}(\boldsymbol{d}, \boldsymbol{w})=(\boldsymbol{L} \boldsymbol{d}, \boldsymbol{w})_{\Sigma}
$$

for all $\boldsymbol{d} \in \boldsymbol{D}(\boldsymbol{L})$ and $\boldsymbol{w} \in \boldsymbol{W}$. We assume $a^{\mathrm{s}}$ to be symmetric, coercive and continuous on $\boldsymbol{W}$ with associated norm $\|\boldsymbol{w}\|_{\mathrm{s}} \stackrel{\text { def }}{=}\left(a^{\mathrm{s}}(\boldsymbol{w}, \boldsymbol{w})\right)^{\frac{1}{2}}$. The weak form of the linear coupled problem (1)-(2) reads as follows: for $t>0$, find $(\boldsymbol{u}(t), p(t), \boldsymbol{d}(t), \dot{\boldsymbol{d}}(t)) \in \boldsymbol{V} \times Q \times \boldsymbol{W} \times \boldsymbol{W}$ such that

$$
\left\{\begin{array}{l}
\left.\boldsymbol{u}_{1}\right|_{\Sigma}=\left.\boldsymbol{u}_{2}\right|_{\Sigma}=\dot{\boldsymbol{d}}, \quad \dot{\boldsymbol{d}}=\partial_{t} \boldsymbol{d}, \\
\rho^{\mathrm{f}}\left(\partial_{t} \boldsymbol{u}, \boldsymbol{v}\right)_{\Omega}+a_{\Omega}^{\mathrm{f}}((\boldsymbol{u}, p),(\boldsymbol{v}, q))+\rho^{\mathrm{s}} \epsilon\left(\partial_{t} \dot{\boldsymbol{d}}, \boldsymbol{w}\right)_{\Sigma}+a^{\mathrm{s}}(\boldsymbol{d}, \boldsymbol{w})=0,
\end{array}\right.
$$

for all $(\boldsymbol{v}, q, \boldsymbol{w}) \in \boldsymbol{V} \times Q \times \boldsymbol{W}$ with $\left.\boldsymbol{v}\right|_{\Sigma}=\boldsymbol{w}$. Taking $(\boldsymbol{v}, q, \boldsymbol{w})=(\boldsymbol{u}(t), p(t), \dot{\boldsymbol{d}}(t))$ in $(3)$ we get, for $t>0$, the following standard energy identity

$$
\begin{aligned}
\frac{\rho^{\mathrm{f}}}{2}\|\boldsymbol{u}(t)\|_{0, \Omega}^{2}+\frac{\rho^{\mathrm{s}} \epsilon}{2}\|\dot{\boldsymbol{d}}(t)\|_{0, \Sigma}^{2}+\frac{1}{2}\|\boldsymbol{d}(t)\|_{\mathrm{s}}^{2}+2 \mu \int_{0}^{t}\|\boldsymbol{\epsilon}(\boldsymbol{u}(s))\|_{0, \Omega}^{2} \mathrm{~d} s & \\
& =\frac{\rho^{\mathrm{f}}}{2}\left\|\boldsymbol{u}_{0}\right\|_{0, \Omega}^{2}+\frac{\rho^{\mathrm{s}}}{2}\left\|\dot{\boldsymbol{d}}_{0}\right\|_{0, \Sigma}^{2}+\frac{1}{2}\left\|\boldsymbol{d}_{0}\right\|_{\mathrm{s}}^{2} .
\end{aligned}
$$

\section{$2.1 \quad$ Space semi-discretization}

The dynamic relation $\left(22_{2}\right.$ introduces jumps in the fluid pressure and in the velocity gradient across the interface $\Sigma$. We propose to approximate the fluid velocity and pressure on triangulations of $\Omega$ which are independent of the interface $\Sigma$ (see Figure 2(a)). In order to guarantee the optimality of the approximations, we allow the discrete fluid solution to be discontinuous inside the elements which are intersected by the interface. In this unfitted framework, the interface coupling conditions $(2)_{1,2}$ will be enforced through a Nitsche's type mortaring.

\subsubsection{Nitsche-XFEM semi-discrete formulation}

For the sake of simplicity, we assume that $\Omega$ and $\Sigma$ are polyhedral. For the construction of the discrete approximation spaces in the fluid, we follow the unfitted approach reported in [42, 6]. To this purpose, we consider two family of meshes $\left\{\mathcal{T}_{i, h}^{\mathrm{f}}\right\}_{0<h<1}, i=1,2$, where each $\mathcal{T}_{i, h}^{\mathrm{f}}$ covers the fluid region $\Omega_{i}$. Each mesh $\mathcal{T}_{i, h}^{\mathrm{f}}$ is fitted to the exterior boundary $\Gamma_{i}$ but, in general, not to $\Sigma$ (see Figure 2(b)). Moreover, we assume that for every element $K \in \mathcal{T}_{1, h}^{\mathrm{f}} \cap \mathcal{T}_{2, h}^{\mathrm{f}}$ we have $K \cap \Sigma \neq \emptyset$. Note that $\mathcal{T}_{1, h}^{\mathrm{f}} \cup \mathcal{T}_{2, h}^{\mathrm{f}}$ gives a conforming triangulation of the whole fluid domain $\Omega$. We denote by $\Omega_{i, h}$ the domain covered by $\mathcal{T}_{i, h}^{\mathrm{f}}$, viz.,

$$
\Omega_{i, h} \stackrel{\text { def }}{=} \operatorname{int}\left(\cup_{K \in \mathcal{T}_{i, h}^{\mathrm{f}}} K\right) .
$$


Finally, for the solid, we consider a family of triangulations $\left\{\mathcal{T}_{h}^{\mathrm{s}}\right\}_{0<h<1}$ of $\Sigma$. For the sake of simplicity, we assume that the three families of triangulations are quasi-uniform.

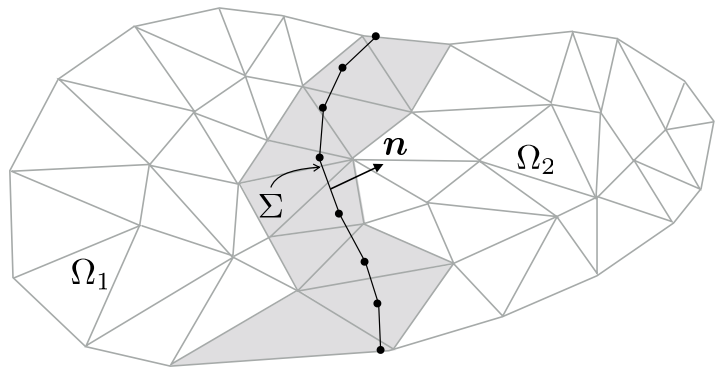

(a)

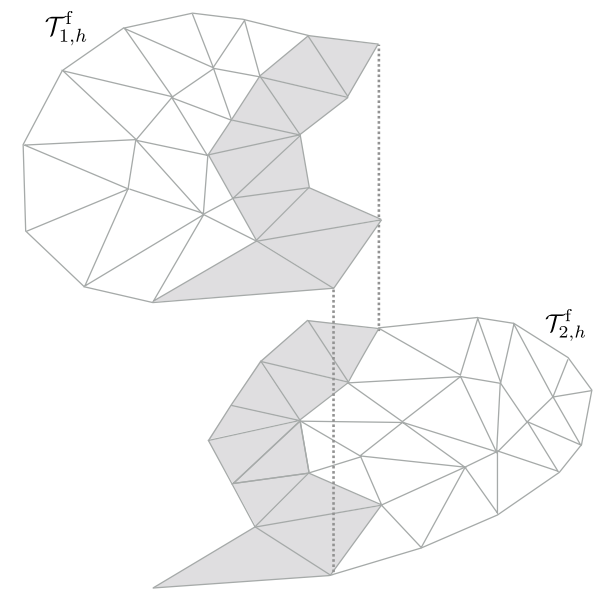

(b)

Figure 2: Unfitted fluid-solid meshes. In grey the elements intersected by $\Sigma$ (a). The triangulations $\mathcal{T}_{1, h}^{\mathrm{f}}$ and $\mathcal{T}_{2, h}^{\mathrm{f}}$, with the overlapping region in grey (b).

We introduce the following standard spaces of continuous piecewise affine functions:

$$
\begin{gathered}
X_{i, h}^{\mathrm{f}} \stackrel{\text { def }}{=}\left\{v_{h} \in C^{0}\left(\overline{\Omega_{i, h}}\right) / v_{h \mid K} \in \mathbb{P}_{1}(K) \quad \forall K \in \mathcal{T}_{i, h}^{\mathrm{f}}\right\}, \\
X_{h}^{\mathrm{s}} \stackrel{\text { def }}{=}\left\{v_{h} \in C^{0}(\bar{\Sigma}) / v_{h \mid K} \in \mathbb{P}_{1}(K) \quad \forall K \in \mathcal{T}_{h}^{\mathrm{s}}\right\} .
\end{gathered}
$$

Associated with $X_{i, h}^{\mathrm{f}}$, we define the spaces

$$
\boldsymbol{V}_{i, h} \stackrel{\text { def }}{=}\left\{\boldsymbol{v}_{i, h} \in\left[X_{i, h}^{\mathrm{f}}\right]^{d} /\left.\boldsymbol{v}_{i, h}\right|_{\Gamma_{i}}=0\right\}, \quad Q_{i, h} \stackrel{\text { def }}{=} X_{i, h}^{\mathrm{f}}, \quad i=1,2 .
$$

For the approximation of the fluid velocity and pressure we will consider the product spaces $\boldsymbol{V}_{h} \stackrel{\text { def }}{=} \boldsymbol{V}_{1, h} \times \boldsymbol{V}_{2, h}$ and $Q_{h} \stackrel{\text { def }}{=} Q_{1, h} \times Q_{2, h}$, respectively. The solid displacement and velocity are approximated in $\boldsymbol{W}_{h} \stackrel{\text { def }}{=}\left[X_{h}^{\text {s }}\right]^{d} \cap \boldsymbol{W}$.

Remark 2.2. Note that the discrete velocity and pressure are two-valued in the overlap interfacial region. Hence, for $f_{h}=\left(f_{1, h}, f_{2, h}\right) \in X_{1, h}^{\mathrm{f}} \times X_{2, h}^{\mathrm{f}}$, the associated function

$$
f_{\Omega, h} \stackrel{\text { def }}{=}\left\{\begin{array}{lll}
\left.f_{1, h}\right|_{\Omega_{1}} & \text { in } & \Omega_{1} \\
\left.f_{2, h}\right|_{\Omega_{2}} & \text { in } & \Omega_{2}
\end{array}\right.
$$

may develop discontinuities across the interface $\Sigma$. This feature is illustrated in Figures 3 and 5. This is one of the fundamental ingredients of the present XFEM approach to approximate the solution of (1)-2.

Since the velocity/pressure discrete spaces $\boldsymbol{V}_{h} / Q_{h}$ fail to satisfy the inf-sup condition, we consider a symmetric pressure stabilization operator $s_{h}: Q_{h} \times Q_{h} \rightarrow \mathbb{R}$, for instance, the classical Brezzi-Pitkäranta method (see, e.g.,. [10])

$$
s_{h}\left(p_{h}, q_{h}\right) \stackrel{\text { def }}{=} \gamma_{\mathrm{p}} \frac{h^{2}}{\mu} \sum_{i=1,2}\left(\nabla p_{i, h}, \nabla q_{i, h}\right)_{\Omega_{i, h}},
$$

$\mathrm{RR} \mathrm{n}^{\circ} 8723$ 


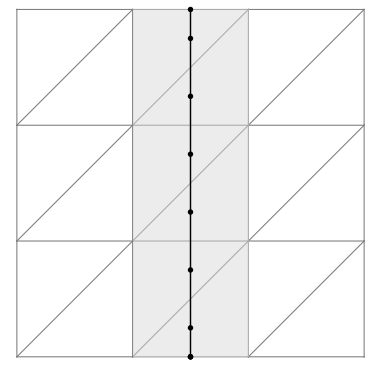

(a) Unfitted meshes

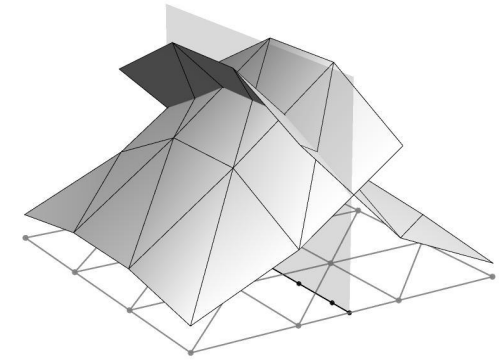

(b) Weak discontinuity

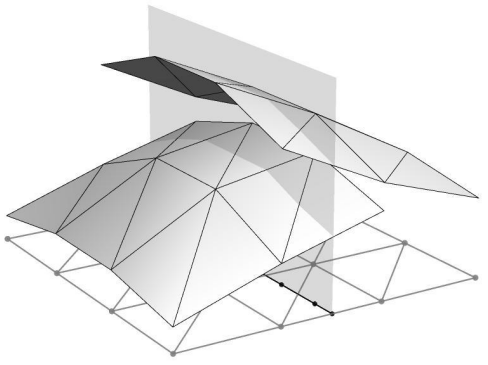

(c) Strong discontinuity

Figure 3: Idealized fully intersected configuration. Example of functions $f_{h} \in X_{1, h}^{\mathrm{f}} \times X_{2, h}^{\mathrm{f}}$.

with $\gamma_{p}>0$ a user-defined parameter. Note that the stabilization acts on the whole computational domain $\Omega_{1, h} \times \Omega_{2, h}$.

In order to guarantee robustness of the method with respect to the way the fluid mesh $\mathcal{T}_{1, h}^{\mathrm{f}} \cup \mathcal{T}_{2, h}^{\mathrm{f}}$ is intersected by the solid mesh $\mathcal{T}_{h}^{\mathrm{s}}$, we consider the ghost-penalty stabilization operator $g_{h}: \boldsymbol{V}_{h} \times \boldsymbol{V}_{h} \rightarrow \mathbb{R}$ defined by

$$
g_{h}\left(\boldsymbol{u}_{h}, \boldsymbol{v}_{h}\right)=\gamma_{\mathrm{g}} \mu h \sum_{i=1}^{2} \sum_{F \in \mathcal{F}_{i, h}^{\Sigma}}\left(\llbracket \nabla \boldsymbol{u}_{i, h} \rrbracket_{F}, \llbracket \nabla \boldsymbol{v}_{i, h} \rrbracket_{F}\right)_{F},
$$

where $\gamma_{\mathrm{g}}>0$ is a user-defined parameter and the symbol $\llbracket \rrbracket_{F}$ denotes the jump across the edge or face $F$ belonging to $\mathcal{F}_{i, h}^{\Sigma}$, the set of interior edges or faces of the elements of $\mathcal{T}_{i, h}^{\mathrm{f}}$ intersected by $\Sigma$, i.e., $F \in \mathcal{F}_{i, h}^{\Sigma}$ if $F$ is a $(d-1)$-manifold and there exist $K_{1}, K_{2} \in \mathcal{T}_{i, h}^{\mathrm{f}}$, with $K_{1} \cap \Sigma \neq \varnothing$ or $K_{2} \cap \Sigma \neq \varnothing$, such that $F=K_{1} \cap K_{2}$. From [12], it follows that this operator extends the natural $H^{1}$-coercivity in the physical domain to the whole $\Omega_{1, h} \times \Omega_{2, h}$, viz.,

$$
\tilde{c}_{\mathrm{S}}\left(\sum_{i=1}^{2} \mu\left\|\boldsymbol{\epsilon}\left(\boldsymbol{v}_{i, h}\right)\right\|_{0, \Omega_{i, h}}^{2}+g_{h}\left(\boldsymbol{v}_{h}, \boldsymbol{v}_{h}\right)\right) \leq \mu \sum_{i=1}^{2}\left\|\boldsymbol{\epsilon}\left(\boldsymbol{v}_{i, h}\right)\right\|_{0, \Omega_{i}}^{2}+g_{h}\left(\boldsymbol{v}_{h}, \boldsymbol{v}_{h}\right),
$$

for all $\boldsymbol{v}_{h} \in \boldsymbol{V}_{h}$, with $\tilde{c}_{\mathrm{s}}>0$ depending on $\gamma_{\mathrm{g}}$. The total stabilization operator is hence given by

$$
S_{h}\left(\left(\boldsymbol{u}_{h}, p_{h}\right),\left(\boldsymbol{v}_{h}, q_{h}\right)\right) \stackrel{\text { def }}{=} s_{h}\left(q_{h}, q_{h}\right)+g_{h}\left(\boldsymbol{u}_{h}, \boldsymbol{v}_{h}\right),
$$

with the associated semi-norm $\left|\left(\boldsymbol{v}_{h}, q_{h}\right)\right|_{S} \stackrel{\text { def }}{=} \sqrt{S_{h}\left(\left(\boldsymbol{v}_{h}, p_{h}\right),\left(\boldsymbol{v}_{h}, q_{h}\right)\right)}$. We also introduce the fluid discrete bi-linear form

$$
a_{h}^{\mathrm{f}}\left(\left(\boldsymbol{u}_{h}, p_{h}\right),\left(\boldsymbol{v}_{h}, q_{h}\right)\right) \stackrel{\text { def }}{=} a_{\Omega_{1} \cup \Omega_{2}}^{\mathrm{f}}\left(\left(\boldsymbol{u}_{h}, p_{h}\right),\left(\boldsymbol{v}_{h}, q_{h}\right)\right)+S_{h}\left(\left(\boldsymbol{u}_{h}, p_{h}\right),\left(\boldsymbol{v}_{h}, q_{h}\right)\right) .
$$

The proposed space semi-discrete approximation of (1)-2 reads as follows: for $t>0$, find

$$
\left(\boldsymbol{u}_{h}(t), p_{h}(t), \dot{\boldsymbol{d}}_{h}(t), \boldsymbol{d}_{h}(t)\right) \in \boldsymbol{V}_{h} \times Q_{h} \times \boldsymbol{W}_{h} \times \boldsymbol{W}_{h},
$$


such that $\dot{\boldsymbol{d}}_{h}(t)=\partial_{t} \boldsymbol{d}_{h}(t)$ and

$$
\begin{aligned}
& \rho^{\mathrm{f}}\left(\partial_{t} \boldsymbol{u}_{h}, \boldsymbol{v}_{h}\right)_{\Omega}+a_{h}^{\mathrm{f}}\left(\left(\boldsymbol{u}_{h}, p_{h}\right),\left(\boldsymbol{v}_{h}, q_{h}\right)\right)+\rho^{\mathrm{s}} \epsilon\left(\partial_{t} \dot{\boldsymbol{d}}_{h}, \boldsymbol{w}_{h}\right)_{\Sigma}+a^{\mathrm{s}}\left(\boldsymbol{d}_{h}, \boldsymbol{w}_{h}\right) \\
& -\sum_{i=1}^{2}\left(\boldsymbol{\sigma}\left(\boldsymbol{u}_{i, h}, p_{i, h}\right) \boldsymbol{n}_{i}, \boldsymbol{v}_{i, h}-\boldsymbol{w}_{h}\right)_{\Sigma}-\sum_{i=1}^{2}\left(\boldsymbol{u}_{i, h}-\dot{\boldsymbol{d}}_{h}, \boldsymbol{\sigma}\left(\boldsymbol{v}_{i, h},-q_{i, h}\right) \boldsymbol{n}_{i}\right)_{\Sigma} \\
& +\frac{\gamma \mu}{h} \sum_{i=1}^{2}\left(\boldsymbol{u}_{i, h}-\dot{\boldsymbol{d}}_{h}, \boldsymbol{v}_{i, h}-\boldsymbol{w}_{h}\right)_{\Sigma}=0
\end{aligned}
$$

for all $\left(\boldsymbol{v}_{h}, q_{h}, \boldsymbol{w}_{h}\right) \in \boldsymbol{V}_{h} \times Q_{h} \times \boldsymbol{W}_{h}$. Here, $\gamma>0$ is a positive parameter given by Lemma 2.1 below (see also Remark 2.5).

Remark 2.3. It should be noted that, unlike [47, Section 4.1], the interface fluid tractions from opposite sides do not cancel in (9). This is a direct consequence of the XFEM nature of the velocity/pressure space $\boldsymbol{V}_{h} \times Q_{h}$, which guarantees the strong consistency of (9) with (1) - (2).

Remark 2.4. In the case of a non-polyhedral interface (see Figure 1), $\Sigma$ and $a^{\mathrm{s}}(\cdot, \cdot)$ in (9) have to be replaced by their corresponding $h$-dependent approximations $\Sigma_{h}$ and $a_{h}^{\mathrm{s}}(\cdot, \cdot)$, respectively.

The above space semi-discretized formulation can be viewed as an extension of the unfitted method recently introduced in [18] to the case of coupling with immersed thin-walled structures. In the next section, we will build on this relation to briefly discuss the stability and convergence properties of $(9)$.

\subsubsection{Stability and convergence}

The next lemma exploits property (7) to guarantee the coercivity of the Stokes-Nitsche's operator in $(9)$.

Lemma 2.1. For $\gamma>0$ sufficiently large, there exists a constant $c_{\mathrm{s}}>0$ such that

$$
\begin{aligned}
c_{\mathrm{S}} & \left(\mu \sum_{i=1}^{2}\left\|\boldsymbol{\nabla} \boldsymbol{v}_{i, h}\right\|_{0, \Omega_{i, h}}^{2}+\frac{\gamma \mu}{h} \sum_{i=1}^{2}\left\|\boldsymbol{v}_{i, h}-\boldsymbol{w}_{h}\right\|_{0, \Sigma}^{2}+\left|\left(\boldsymbol{v}_{h}, q_{h}\right)\right|_{S}^{2}\right) \\
\leq & a_{h}^{\mathrm{f}}\left(\left(\boldsymbol{v}_{h}, q_{h}\right),\left(\boldsymbol{v}_{h}, q_{h}\right)\right)-\sum_{i=1}^{2}\left(\boldsymbol{\sigma}\left(\boldsymbol{v}_{i, h}, q_{i, h}\right) \boldsymbol{n}_{i}, \boldsymbol{v}_{i, h}-\boldsymbol{w}_{h}\right)_{\Sigma} \\
& -\sum_{i=1}^{2}\left(\boldsymbol{v}_{i, h}-\boldsymbol{w}_{h}, \boldsymbol{\sigma}\left(\boldsymbol{v}_{i, h},-q_{i, h}\right) \boldsymbol{n}_{i}\right)_{\Sigma}+\frac{\gamma \mu}{h} \sum_{i=1}^{2}\left\|\boldsymbol{v}_{i, h}-\boldsymbol{w}_{h}\right\|_{0, \Sigma}^{2}+\left|\left(\boldsymbol{v}_{h}, q_{h}\right)\right|_{S}^{2}
\end{aligned}
$$

for all $\left(\boldsymbol{v}_{h}, q_{h}, \boldsymbol{w}_{h}\right) \in \boldsymbol{V}_{h} \times Q_{h} \times \boldsymbol{W}_{h}$. 
Proof. The proof follows the arguments reported in [18, Lemma 3.1]. First, we have

$$
\begin{aligned}
& a_{h}^{\mathrm{f}}\left(\left(\boldsymbol{v}_{h}, q_{h}\right),\left(\boldsymbol{v}_{h}, q_{h}\right)\right)-\sum_{i=1}^{2}\left(\boldsymbol{\sigma}\left(\boldsymbol{v}_{i, h}, q_{i, h}\right) \boldsymbol{n}_{i}, \boldsymbol{v}_{i, h}-\boldsymbol{w}_{h}\right)_{\Sigma} \\
& \quad-\sum_{i=1}^{2}\left(\boldsymbol{v}_{i, h}-\boldsymbol{w}_{h}, \boldsymbol{\sigma}\left(\boldsymbol{v}_{i, h},-q_{i, h}\right) \boldsymbol{n}_{i}\right)_{\Sigma}+\frac{\gamma \mu}{h} \sum_{i=1}^{2}\left\|\boldsymbol{v}_{i, h}-\boldsymbol{w}_{h}\right\|_{0, \Sigma}^{2}+\left|\left(\boldsymbol{v}_{h}, q_{h}\right)\right|_{S}^{2} \\
& =2 \mu \sum_{i=1}^{2}\left\|\boldsymbol{\epsilon}\left(\boldsymbol{v}_{i, h}\right)\right\|_{0, \Omega_{i}}^{2}-2 \sum_{i=1}^{2}\left(\boldsymbol{\sigma}\left(\boldsymbol{v}_{i, h}, 0\right) \boldsymbol{n}, \boldsymbol{v}_{i, h}-\boldsymbol{w}_{h}\right)_{\Sigma} \\
& \quad+\sum_{i=1}^{2} \frac{\gamma \mu}{h}\left\|\boldsymbol{v}_{i, h}-\boldsymbol{w}_{h}\right\|_{0, \Sigma}^{2}+\left|\left(\boldsymbol{v}_{h}, q_{h}\right)\right|_{S}^{2} .
\end{aligned}
$$

Combining the Cauchy-Schwarz inequality with the following (robust) trace inequality

$$
h\left\|\boldsymbol{\epsilon}\left(\boldsymbol{v}_{i, h}\right) \boldsymbol{n}\right\|_{0, \Sigma}^{2} \leq C_{\mathrm{T}}\left\|\boldsymbol{\epsilon}\left(\boldsymbol{v}_{i, h}\right)\right\|_{0, \Omega_{i, h}}^{2} \quad \forall \boldsymbol{v}_{i, h} \in \boldsymbol{V}_{i, h}, \quad i=1,2,
$$

with $C_{\mathrm{T}}>0$, we have

$$
\sum_{i=1}^{2}\left(2 \boldsymbol{\sigma}\left(\boldsymbol{v}_{i, h}, 0\right) \boldsymbol{n}, \boldsymbol{v}_{i, h}-\boldsymbol{w}_{h}\right)_{\Sigma} \leq \frac{8 C_{\mathrm{T}}}{\gamma} \mu \sum_{i=1}^{2}\left\|\boldsymbol{\epsilon}\left(\boldsymbol{v}_{i, h}\right)\right\|_{0, \Omega_{i, h}}^{2}+\frac{1}{2} \sum_{i=1}^{2} \frac{\gamma \mu}{h}\left\|\boldsymbol{v}_{i, h}-\boldsymbol{w}_{h}\right\|_{0, \Sigma}^{2} .
$$

We conclude by using the strengthened stability 7 provided by the ghost-penalty operator , taking

$$
\gamma>\frac{8 C_{\mathrm{T}}}{\tilde{c}_{\mathrm{s}}}
$$

and using Korn's inequality.

Remark 2.5. The proof of Lemma 2.1 provides a lower bound for the parameter $\gamma>0$ through the relation (11). The constants therein can be estimated automatically from the solution of generalized eigenvalue problems associated with the inequalities (7) and 100. We refer to 45, Section 3] for an overview of this approach, and to [61, 28] for applications in the context of Nitsche's method.

If we take $\boldsymbol{v}_{h}=\boldsymbol{u}_{h}(t), q_{h}=p_{h}(t)$ and $\boldsymbol{w}_{h}=\dot{\boldsymbol{d}}_{h}(t)$ in (9) and apply the result of Lemma 2.1 we get the following discrete counterpart of (4):

$$
\begin{aligned}
& \frac{\rho^{\mathrm{f}}}{2}\left\|\boldsymbol{u}_{h}(t)\right\|_{0, \Omega}^{2}+\frac{\rho^{\mathrm{s}}}{2}\left\|\dot{\boldsymbol{d}}_{h}(t)\right\|_{0, \Sigma}^{2}+\frac{1}{2}\left\|\boldsymbol{d}_{h}(t)\right\|_{\mathrm{s}}^{2} \\
&+c_{\mathrm{s}} \int_{0}^{t}\left(\sum_{i=1}^{2} \mu\left\|\boldsymbol{\nabla} \boldsymbol{u}_{i, h}(s)\right\|_{0, \Omega_{i, h}}^{2}+\sum_{i=1}^{2} \frac{\gamma \mu}{h}\left\|\boldsymbol{u}_{i, h}(s)-\dot{\boldsymbol{d}}_{h}(s)\right\|_{0, \Sigma}^{2}+\left|\left(\boldsymbol{u}_{h}(s), p_{h}(s)\right)\right|_{S}^{2}\right) \mathrm{d} s \\
& \quad \leq \frac{\rho^{\mathrm{f}}}{2}\left\|\boldsymbol{u}_{h}(0)\right\|_{0, \Omega}^{2}+\frac{\rho^{\mathrm{s}}}{2}\left\|\dot{\boldsymbol{d}}_{h}(0)\right\|_{0, \Sigma}^{2}+\frac{1}{2}\left\|\boldsymbol{d}_{h}(0)\right\|_{\mathrm{s}}^{2}
\end{aligned}
$$

which guarantees the energy stability of $(9)$.

The following result states the optimal accuracy of $(9)$ in the energy norm, under regularity assumptions on the solution of (1)-22. The symbol $\lesssim$ denotes inequality up to a multiplicative constant independent of $h$. 
Theorem 2.1. Let $(\boldsymbol{u}, p, \dot{\boldsymbol{d}}, \boldsymbol{d})$ be the solution of (1)-2) and $\left(\boldsymbol{u}_{h}, p_{h}, \dot{\boldsymbol{d}}_{h}, \boldsymbol{d}_{h}\right)$ be given by (9). We assume that the interface $\Sigma$ is flat and that

$$
\boldsymbol{u} \in\left[H^{1}\left(\mathbb{R}^{+} ; H^{2}\left(\Omega_{1} \cup \Omega_{2}\right)\right)\right]^{d}, p \in C^{0}\left(\mathbb{R}^{+} ; H^{1}\left(\Omega_{1} \cup \Omega_{2}\right)\right), \boldsymbol{d}, \dot{\boldsymbol{d}} \in\left[H^{1}\left(\mathbb{R}^{+} ; H^{2}(\Sigma)\right)\right]^{d} .
$$

For $t>0$, there holds:

$$
\begin{aligned}
\frac{\rho^{\mathrm{f}}}{2}\left\|\left(\boldsymbol{u}_{h}-\boldsymbol{u}\right)(t)\right\|_{0, \Omega}^{2} & +\frac{\rho^{\mathrm{s}}}{2}\left\|\left(\dot{\boldsymbol{d}}_{h}-\dot{\boldsymbol{d}}\right)(t)\right\|_{0, \Sigma}^{2}+\frac{1}{2}\left\|\left(\boldsymbol{d}_{h}-\boldsymbol{d}\right)(t)\right\|_{\mathrm{s}}^{2} \\
& +c_{\mathrm{s}} \int_{0}^{t} \sum_{i=1}^{2}\left(\mu\left\|\nabla\left(\boldsymbol{u}_{i, h}-\boldsymbol{u}\right)(s)\right\|_{0, \Omega_{i}}^{2}+\frac{\gamma \mu}{h}\left\|\left(\boldsymbol{u}_{i, h}-\dot{\boldsymbol{d}}_{i, h}\right)(s)\right\|_{0, \Sigma}^{2}\right) \mathrm{d} s \lesssim h^{2},
\end{aligned}
$$

where $\gamma>0$ is given by Lemma 2.1.

Proof. The result follows by applying the arguments presented in the proof of [18, Theorem 3.1] to each $\Omega_{i, h}, i=1,2$.

\subsubsection{Partially intersected fluid domain}

In this section we discuss how the space semi-discrete formulation (9) can be generalized to the case in which the interface $\Sigma$ only partially intersects the domain $\Omega$ (see Figure 4(a)). In order to set up the new discrete spaces $\boldsymbol{V}_{h}$ and $Q_{h}$ for the fluid, we consider a fictitious prolongation of $\Sigma$, denoted by $\Sigma_{h}^{\text {tip }}$, so that $\Sigma \cup \Sigma_{h}^{\text {tip }}$ divides $\Omega$ into two open domains (see Figure 4 (a)). The fluid-fluid fictitious interface is defined in terms of the partition $\Sigma_{h}^{\text {tip }}=\widetilde{\Sigma}_{h} \cup \widehat{\Sigma}_{h}$ where:

- The part $\widetilde{\Sigma}_{h}$ is included in the set of elements containing the tip of $\Sigma$. Moreover, within each of these elements, it is defined as the prolongation of the interface up to the point which is opposite to the edge or face intersected by $\Sigma$.

- The part $\widehat{\Sigma}_{h}$ is aligned with the edges or faces of the mesh.

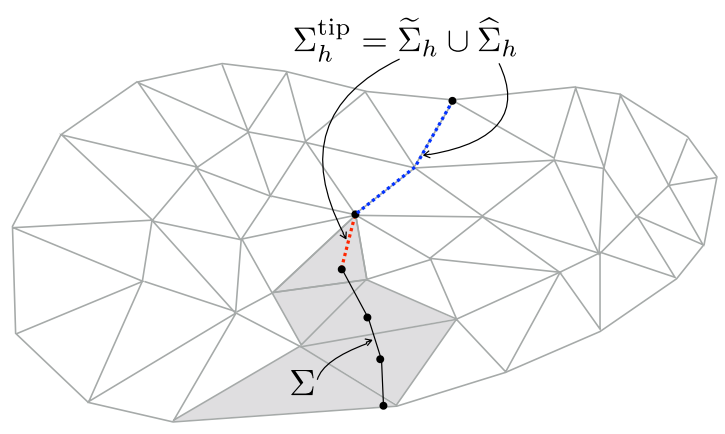

(a)

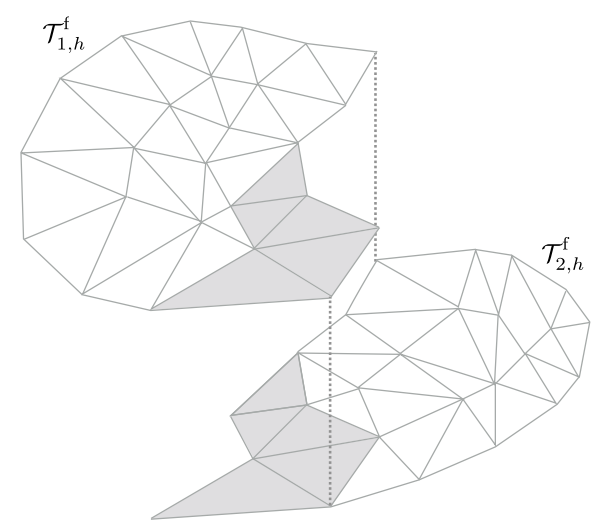

(b)

Figure 4: The fluid-solid interface $\Sigma$ and the fluid-fluid fictitious interface $\tilde{\Sigma}_{h}$ in red (a). The two new triangulations with the overlapping region in grey (b). 


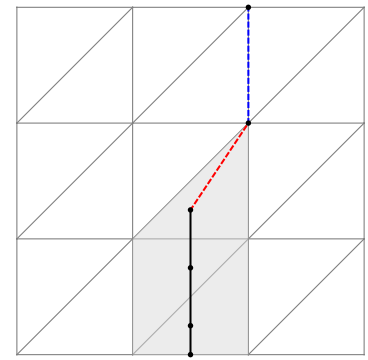

(a) Unfitted meshes

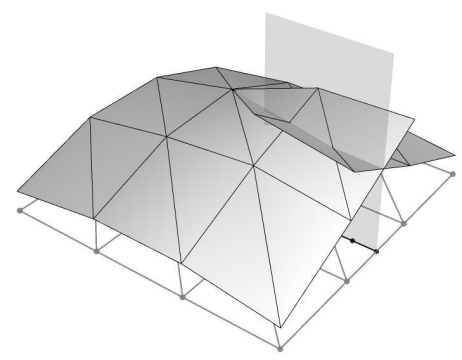

(b) Weak discontinuity

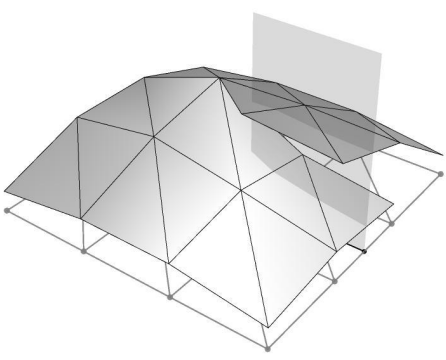

(c) Strong discontinuity

Figure 5: Idealized partially intersected configuration (the red dotted line represents the fictitious fluid-fluid interface). Example of functions $f_{h} \in X_{1, h}^{\mathrm{f}} \times X_{2, h}^{\mathrm{f}}$.

We now proceed, as in Section 2.1.1 by introducing two overlapping triangulations $\mathcal{T}_{i, h}^{\mathrm{f}}$, $i=1,2$ (see Figure 4(b)). Note that the overlap region reduces to the set of elements intersected by $\Sigma$. The associated discrete spaces $X_{i, h}^{\mathrm{f}}, i=1,2$, are then defined as in (5). At last, the fluid velocity and pressure spaces are derived from (6) by strongly enforcing the continuity of the velocity and pressure across $\widehat{\Sigma}_{h}$ (see Figure 5), viz.:

$$
\begin{gathered}
\boldsymbol{V}_{h} \stackrel{\text { def }}{=}\left\{\boldsymbol{v}_{h}=\left(\boldsymbol{v}_{1, h}, \boldsymbol{v}_{2, h}\right) \in \boldsymbol{V}_{1, h} \times \boldsymbol{V}_{2, h} / \boldsymbol{v}_{1, h}=\boldsymbol{v}_{2, h} \text { on } \widehat{\Sigma}_{h}\right\}, \\
Q_{h} \stackrel{\text { def }}{=}\left\{q_{h}=\left(q_{1, h}, q_{2, h}\right) \in Q_{1, h} \times Q_{2, h} / q_{1, h}=q_{2, h} \text { on } \widehat{\Sigma}_{h}\right\} .
\end{gathered}
$$

The functions of these spaces are continuous in the domain

$$
\Omega_{h} \stackrel{\text { def }}{=} \Omega \backslash\left(\Sigma \cup \widetilde{\Sigma}_{h}\right),
$$

but discontinuous across the interface $\Sigma \cup \widetilde{\Sigma}_{h}$. The fluid discrete bi-linear form (8) is hence redefined as

$$
a_{h}^{\mathrm{f}}\left(\left(\boldsymbol{u}_{h}, p_{h}\right),\left(\boldsymbol{v}_{h}, q_{h}\right)\right) \stackrel{\text { def }}{=} a_{\Omega_{h}}^{\mathrm{f}}\left(\left(\boldsymbol{u}_{h}, p_{h}\right),\left(\boldsymbol{v}_{h}, q_{h}\right)\right)+S_{h}\left(\left(\boldsymbol{u}_{h}, p_{h}\right),\left(\boldsymbol{v}_{h}, q_{h}\right)\right) .
$$

The continuity of velocity and stress across the fictitious fluid-fluid interface $\widetilde{\Sigma}_{h}$ will be enforced in a consistent weak fashion, using Nitsche's method (see, e.g., [25, Section 6.1.2]). In summary, the resulting semi-discrete approximation of (1)-2 reads as follows: for $t>0$, find

$$
\left(\boldsymbol{u}_{h}(t), p_{h}(t), \dot{\boldsymbol{d}}_{h}(t), \boldsymbol{d}_{h}(t)\right) \in \boldsymbol{V}_{h} \times Q_{h} \times \boldsymbol{W}_{h} \times \boldsymbol{W}_{h},
$$

such that $\dot{\boldsymbol{d}}_{h}(t)=\partial_{t} \boldsymbol{d}_{h}(t)$ and

$$
\begin{aligned}
& \rho^{\mathrm{f}}\left(\partial_{t} \boldsymbol{u}_{h}, \boldsymbol{v}_{h}\right)_{\Omega}+a_{h}^{\mathrm{f}}\left(\left(\boldsymbol{u}_{h}, p_{h}\right),\left(\boldsymbol{v}_{h}, q_{h}\right)\right)+\rho^{\mathrm{s}} \epsilon\left(\partial_{t} \dot{\boldsymbol{d}}_{h}, \boldsymbol{w}_{h}\right)_{\Sigma}+a^{\mathrm{s}}\left(\boldsymbol{d}_{h}, \boldsymbol{w}_{h}\right) \\
& -\sum_{i=1}^{2}\left(\boldsymbol{\sigma}\left(\boldsymbol{u}_{i, h}, p_{i, h}\right) \boldsymbol{n}_{i}, \boldsymbol{v}_{i, h}-\boldsymbol{w}_{h}\right)_{\Sigma}-\sum_{i=1}^{2}\left(\boldsymbol{u}_{i, h}-\dot{\boldsymbol{d}}_{h}, \boldsymbol{\sigma}\left(\boldsymbol{v}_{i, h},-q_{i, h}\right) \boldsymbol{n}_{i}\right)_{\Sigma} \\
& +\frac{\gamma \mu}{h} \sum_{i=1}^{2}\left(\boldsymbol{u}_{i, h}-\dot{\boldsymbol{d}}_{h}, \boldsymbol{v}_{i, h}-\boldsymbol{w}_{h}\right)_{\Sigma}-\left(\left\{\boldsymbol{\sigma}\left(\boldsymbol{u}_{h}, p_{h}\right)\right\} \boldsymbol{n}, \llbracket \boldsymbol{v}_{h} \rrbracket\right)_{\widetilde{\Sigma}_{h}} \\
& -\left(\left\{\boldsymbol{\sigma}\left(\boldsymbol{v}_{h},-q_{h}\right)\right\} \boldsymbol{n}, \llbracket \boldsymbol{u}_{h} \rrbracket\right)_{\widetilde{\Sigma}_{h}}+\frac{\gamma \mu}{h}\left(\llbracket \boldsymbol{u}_{h} \rrbracket, \llbracket \boldsymbol{v}_{h} \rrbracket\right)_{\widetilde{\Sigma}_{h}}=0
\end{aligned}
$$


for all $\left(\boldsymbol{v}_{h}, q_{h}, \boldsymbol{w}_{h}\right) \in \boldsymbol{V}_{h} \times Q_{h} \times \boldsymbol{W}_{h}$.

Remark 2.6. It should be noted that the sole differences between (9) and (14) are the definition of the discrete fluid space $\boldsymbol{V}_{h} \times Q_{h}$ and the three additional terms acting on the fictitious fluidfluid interface $\widetilde{\Sigma}_{h}$.

Standard arguments show that the energy stability 12 also holds for $(14)$. The extension of Theorem 2.1 is more delicate due to the lack of regularity in the vicinity of the interface tip (see, e.g. [26]).

\subsubsection{Mesh intersection and integrals over cut-elements}

The bilinear forms of the space semi-discrete formulations (9) and (14) require the evaluation of integrals over cut-elements. This is a consequence of the fact that, for consistency, the fluid equations are integrated only in the physical zone of $\Omega_{i, h}, i=1,2$. This is a non-standard implementation problem which demands a specific track of the interface intersections (see, e.g., [53]), namely:

- evaluation of the intersections between the unfitted fluid and solid meshes, i.e., the computation of the cut-elements;

- evaluation of the integrals over the cut-elements.

This can be challenging in practice, particularly if (as in the present framework) the unfitted fluid and solid meshes are unstructured. Regarding the first point, a few algorithms have been recently reported in the literature (see, e.g., [54, 71, 53]). The second is usually faced by sub-dividing the cut-elements into sub-elements for the purpose of the numerical integration (see, e.g., 34, 54]). Such subdivision can however be involved in 3D (particularly for general unstructured meshes). An alternative is the use of the divergence theorem to obtain a boundary representation of the integrals (see, e.g., [53, 66]).

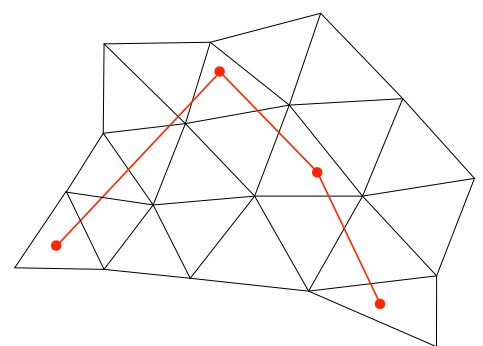

(a)

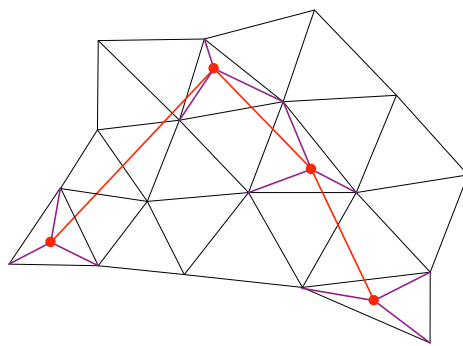

(b)

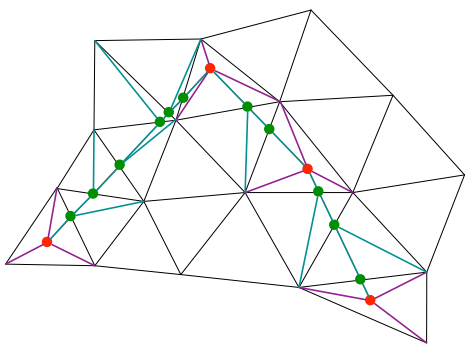

(c)

Figure 6: Main steps of the mesh intersection algorithm: (a) initial state where the solid mesh (in red) and the fluid mesh are not fitted; (b) end of step 1 where the solid mesh vertices have been inserted inside the fluid mesh; (c) end of step 2 where the solid mesh edges have been enforced inside the fluid mesh by a partitioning method. The resulting mesh is the intersected mesh where both meshes are fitted.

In the 2D numerical examples of Section 4 , the above operations have been performed with a customized 2D algorithm which simultaneously yields the mesh intersections and the cut-elements subdivisions (see Figure 67. Its main steps are the following: 
1. We first localize all the solid mesh vertices inside the fluid mesh. This is carried out using a barycentric coordinates based algorithm, which efficiently identifies the element of the fluid mesh containing a given point (see, e.g., [33, Section 2.10] or [2, Section 3] for details). Once localized, the solid vertices are inserted into the fluid mesh. For efficiency, simple insertion patterns are used, instead of complex vertex insertion operators (such as the Delaunay kernel [33]). In this regard, it is worth recalling that the quality of the intersected mesh is definitely not a concern here, whose sole purpose is numerical quadrature in cut-elements (not interpolation). Therefore, simple mesh validity suffices. When a point is inserted into a $2 \mathrm{D}$ mesh composed of triangles, three cases may arise (two of them being degenerated):

- the point falls inside a triangle, then the triangle is split into three triangles;

- the point is on a mesh edge, then the edge is split into two and the two triangles sharing this edge are split into two triangles;

- the point coincides with an existing mesh vertex, then nothing is done.

This step is illustrated in Figures 6(a)-6(b).

2. We consider a partitioning algorithm (see, e.g., [38]) to insert the structure mesh edges into the mesh resulting from step 1. Each edge is treated one at a time. Its endpoints are vertices of the current (intersected) mesh thanks to step 1. Let $A B$ be an edge of the solid mesh. Starting from one of its endpoints, say $A$, we seek for the first current mesh edge intersected by $A B$. The intersection point $P_{1}$ is computed (see [2, Section 5] for details) and inserted into the current mesh. The two triangles sharing the intersected edge containing $P_{1}$ are split into two triangles. At this stage, part of edge $A B$ (i.e., the segment $A P_{1}$ ) has been added to the current mesh. Then, the process is pursued by seeking for the intersection between sub-edge $P_{1} B$ and the current mesh edges, which will give a new intersection point $P_{2}$ and so on. If after $n$ intersection steps the sub-edge $P_{n} B$ belongs to the current mesh, the whole solid edge $A B$ has been inserted into this mesh. It should be noted that, for efficiency, this algorithm works only locally and progresses only through neighboring elements. This step is illustrated in Figures 6(b)-6(c).

Once the mesh intersection has been resolved via the above two steps, the data structures needed to evaluate the terms of the Nitsche-XFEM formulation (9) and (14) are updated accordingly.

\subsection{Time discretization: fully discrete schemes}

This section is devoted to the time discretization of the unfitted spatial semi-discrete formulations $(9)$ and (14). In order to simplify the presentation, we mainly consider the case of (9). The extension to (14) simply follows by adding suitable fluid terms (see Remark 2.6). Several coupling strategies will be discussed, which differ in their degree of fluid-solid splitting: implicit, explicit and semi-implicit nature (Algorithms 13 below). The theoretical discussion on the stability and accuracy of the methods introduced will be substantiated by numerical experiments in Section 4.1.

In the succeeding text, $\tau>0$ denotes the time-step length, $t_{n} \stackrel{\text { def }}{=} n \tau$ for $n \in \mathbb{N}$, and $\partial_{\tau} x^{n} \stackrel{\text { def }}{=} \frac{1}{\tau}\left(x^{n}-x^{n-1}\right)$ stands for the first-order backward difference. The superscript ${ }^{n, \star}$ will denote explicit extrapolations of order $r \in\{0,1,2\}$ to $x^{n}$, namely,

$$
x^{n, \star} \stackrel{\text { def }}{=}\left\{\begin{array}{lll}
0 & \text { if } & r=0, \\
x^{n-1} & \text { if } & r=1, \\
2 x^{n-1}-x^{n-2} & \text { if } & r=2 .
\end{array}\right.
$$




\subsubsection{Implicit coupling scheme}

An overall backward Euler time-stepping of (9) yields the implicit coupling scheme reported in Algorithm 1. This guarantees unconditional stability and optimal accuracy. In particular, by denoting

$$
E^{n} \stackrel{\text { def }}{=} \frac{\rho^{\mathrm{f}}}{2}\left\|\boldsymbol{u}_{h}^{n}\right\|_{0, \Omega}^{2}+\frac{\rho^{\mathrm{s}}}{2}\left\|\dot{\boldsymbol{d}}_{h}^{n}\right\|_{0, \Sigma}^{2}+\frac{1}{2}\left\|\boldsymbol{d}_{h}^{n}\right\|_{\mathrm{s}}^{2},
$$

the total energy of the discrete system at time $t_{n}$, and by

$$
\mathcal{E}^{n} \stackrel{\text { def }}{=}\left(\frac{\rho^{\mathrm{f}}}{2}\left\|\boldsymbol{u}_{h}^{n}-\boldsymbol{u}\left(t_{n}\right)\right\|_{0, \Omega}^{2}+\frac{\rho^{\mathrm{s}} \epsilon}{2}\left\|\dot{\boldsymbol{d}}_{h}^{n}-\dot{\boldsymbol{d}}\left(t_{n}\right)\right\|_{0, \Sigma}^{2}+\frac{1}{2}\left\|\boldsymbol{d}_{h}^{n}-\boldsymbol{d}\left(t_{n}\right)\right\|_{\mathrm{s}}^{2}\right)^{\frac{1}{2}},
$$

the energy norm of the approximation error, the following theorem holds. We recall that $\lesssim, \gtrsim$ denote inequalities up to multiplicative constants independent of $h$ and $\tau$.

Theorem 2.2. Let $\left\{\left(\boldsymbol{u}_{h}^{n}, p_{h}^{n}, \dot{\boldsymbol{d}}_{h}^{n}, \boldsymbol{d}_{h}^{n}\right)\right\}_{n \geq 1}$ be the sequence given by Algorithm 1 . Then, for $\gamma>0$ given by Lemma 2.1 and $n \geq 1$, there holds

$$
E^{n} \leq E^{0}
$$

Moreover, under for smooth enough solutions, the following a priori error estimate holds

$$
\mathcal{E}^{n} \lesssim h+\tau
$$

Proof. The first result is a straightforward consequence of Lemma 2.1, after taking $\left(\boldsymbol{v}_{h}, q_{h}, \boldsymbol{w}_{h}\right)=$ $\left(\boldsymbol{u}_{h}^{n}, p_{h}^{n}, \dot{\boldsymbol{d}}_{h}^{n}\right)$ in $(15)$. The a priori error estimate follows by combining the arguments of the proof of Theorem 2.1 with Taylor expansions in time.

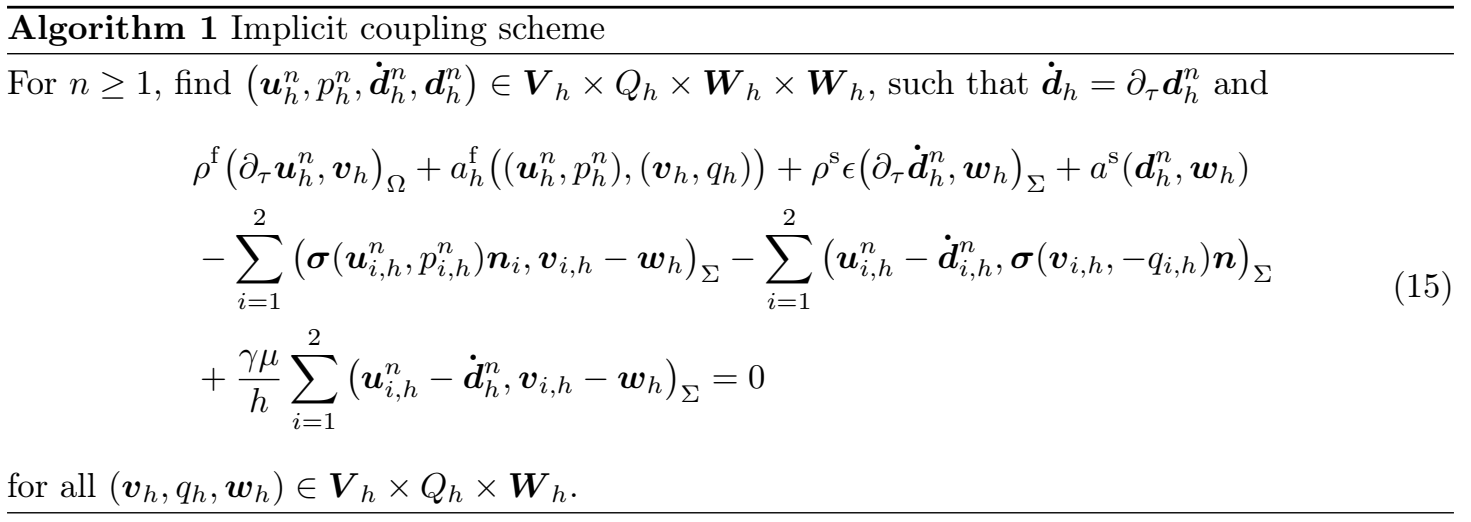

Therefore, due to Theorem 2.2. Algorithm 1 exhibits optimal first-order accuracy (in the energy norm) both in time and space.

Remark 2.7. Second-order accuracy in time can be achieved, for instance, by considering a Crank-Nicolson time-stepping of (9).

The price to pay for the above unconditional stability and accuracy properties of Algorithm 1 . is that, at each time level, the fluid $\left(\boldsymbol{u}_{h}^{n}, p_{h}^{n}\right)$ and solid $\left(\dot{\boldsymbol{d}}_{h}^{n}, \boldsymbol{d}_{h}^{n}\right)$ states are fully coupled, which can be computationally demanding in practice. Indeed, besides the hybrid nature of the coupled problem, general thin-walled solid models discretized by finite elements are known to yield very ill-conditioned stiffness matrices requiring specific solvers (see, e.g., 37]). In the next two sections, we introduce two alternative time discretizations of $(9)$ with a certain degree of splitting between the time-stepping of the fluid and of the solid. 


\subsubsection{Stabilized explicit schemes}

We consider the stabilized explicit coupling paradigm originally introduced in [15, 17, for fitted meshes, and extended in [18] to the unfitted framework. We apply here those ideas to the Nitsche-XFEM spatial semi-discrete formulation (9). To this purpose, we first formulate (9) in terms of two coupled sub-problems by successively taking $\left(\boldsymbol{v}_{h}, q_{h}\right)=(\mathbf{0}, 0)$ and $\boldsymbol{w}_{h}=\mathbf{0}$ in (9). This yields:

- Solid sub-problem:

$$
\begin{aligned}
\rho^{\mathrm{s}} \epsilon\left(\partial_{t} \dot{\boldsymbol{d}}_{h}, \boldsymbol{w}_{h}\right)_{\Sigma}+a^{\mathrm{s}}\left(\boldsymbol{d}_{h}, \boldsymbol{w}_{h}\right)+\left(\llbracket \boldsymbol{\sigma}\left(\boldsymbol{u}_{h}, p_{h}\right) \boldsymbol{n} \rrbracket, \boldsymbol{w}_{h}\right)_{\Sigma} & \\
& +\frac{2 \gamma \mu}{h}\left(\dot{\boldsymbol{d}}_{h}-\left\{\boldsymbol{u}_{h}\right\}, \boldsymbol{w}_{h}\right)_{\Sigma}=0
\end{aligned}
$$

for all $\boldsymbol{w}_{h} \in \boldsymbol{W}_{h}$.

- Fluid sub-problem:

$$
\begin{aligned}
\rho^{\mathrm{f}}\left(\partial_{t} \boldsymbol{u}_{h}, \boldsymbol{v}_{h}\right)_{\Omega} & +a_{h}^{\mathrm{f}}\left(\left(\boldsymbol{u}_{h}, p_{h}\right),\left(\boldsymbol{v}_{h}, q_{h}\right)\right)-\sum_{i=1}^{2}\left(\boldsymbol{\sigma}\left(\boldsymbol{u}_{i, h}, p_{i, h}\right) \boldsymbol{n}_{i}, \boldsymbol{v}_{i, h}\right)_{\Sigma} \\
& -\sum_{i=1}^{2}\left(\boldsymbol{u}_{i, h}-\dot{\boldsymbol{d}}_{h}, \boldsymbol{\sigma}\left(\boldsymbol{v}_{i, h},-q_{i, h}\right) \boldsymbol{n}_{i}\right)_{\Sigma}+\frac{\gamma \mu}{h} \sum_{i=1}^{2}\left(\boldsymbol{u}_{i, h}-\dot{\boldsymbol{d}}_{h}, \boldsymbol{v}_{i, h}\right)_{\Sigma}=0
\end{aligned}
$$

for all $\left(\boldsymbol{v}_{h}, q_{h}\right) \in \boldsymbol{V}_{h} \times Q_{h}$.

We combine a backward Euler time-stepping of the fluid and solid bulk terms in (16)-(17) with an explicit treatment of the interface coupling terms. More precisely, for $n \geq 1$, we have:

1. Solid sub-step: find $\left(\dot{\boldsymbol{d}}_{h}^{n}, \boldsymbol{d}_{h}^{n}\right) \in \boldsymbol{W}_{h} \times \boldsymbol{W}_{h}$ with $\dot{\boldsymbol{d}}_{h}^{n}=\partial_{\tau} \boldsymbol{d}_{h}^{n}$ and such that

$$
\begin{aligned}
\rho^{\mathrm{s}} \epsilon\left(\partial_{\tau} \dot{\boldsymbol{d}}_{h}^{n}, \boldsymbol{w}_{h}\right)_{\Sigma}+a^{\mathrm{s}}\left(\boldsymbol{d}_{h}^{n}, \boldsymbol{w}_{h}\right) & +\frac{2 \gamma \mu}{h}\left(\dot{\boldsymbol{d}}_{h}^{n}, \boldsymbol{w}_{h}\right)_{\Sigma} \\
& \left.=\frac{2 \gamma \mu}{h}\left(\llbracket \boldsymbol{u}_{h}^{n-1}\right\}, \boldsymbol{w}_{h}\right)_{\Sigma}-\left(\llbracket \boldsymbol{\sigma}\left(\boldsymbol{u}_{h}^{n-1}, p_{h}^{n-1}\right) \boldsymbol{n} \rrbracket, \boldsymbol{w}_{h}\right)_{\Sigma}
\end{aligned}
$$

for all $\boldsymbol{w}_{h} \in \boldsymbol{W}_{h}$.

2. Fluid sub-step: find $\left(\boldsymbol{u}_{h}^{n}, p_{h}^{n}\right) \in \boldsymbol{V}_{h} \times Q_{h}$ such that

$$
\begin{aligned}
\rho^{\mathrm{f}}\left(\partial_{\tau} \boldsymbol{u}_{h}^{n}, \boldsymbol{v}_{h}\right)_{\Omega}+a_{h}^{\mathrm{f}}\left(\left(\boldsymbol{u}_{h}^{n}, p_{h}^{n}\right),\left(\boldsymbol{v}_{h}, q_{h}\right)\right)+\frac{\gamma \mu}{h} \sum_{i=1}^{2}\left(\boldsymbol{u}_{i, h}^{n}, \boldsymbol{v}_{i, h}\right)_{\Sigma} \\
-\sum_{i=1}^{2}\left(\boldsymbol{u}_{i, h}^{n}-\dot{\boldsymbol{d}}_{h}^{n}, \boldsymbol{\sigma}\left(\boldsymbol{v}_{i, h},-q_{i, h}\right) \boldsymbol{n}_{i}\right)_{\Sigma}+\frac{\gamma_{0} h}{\gamma \mu} \sum_{i=1}^{2}\left(p_{i, h}^{n}-p_{i, h}^{n-1}, q_{i, h}\right)_{\Sigma} \\
=\frac{\gamma \mu}{h} \sum_{i=1}^{2}\left(\dot{\boldsymbol{d}}_{h}^{n}, \boldsymbol{v}_{i, h}\right)_{\Sigma}+\sum_{i=1}^{2}\left(\boldsymbol{\sigma}\left(\boldsymbol{u}_{i, h}^{n-1}, p_{i, h}^{n-1}\right) \boldsymbol{n}_{i}, \boldsymbol{v}_{i, h}\right)_{\Sigma}
\end{aligned}
$$

for all $\left(\boldsymbol{v}_{h}, q_{h}\right) \in \boldsymbol{V}_{h} \times Q_{h}$. 
A salient feature of this approach is that explicit treatment of the interface coupling in 18 uncouples the computation of $\left(\dot{\boldsymbol{d}}_{h}^{n}, \boldsymbol{d}_{h}^{n}\right)$ and $\left(\boldsymbol{u}_{h}^{n}, p_{h}^{n}\right)$. The fundamental ingredient for the stabitity of the scheme is the weakly consistent term

$$
\frac{\gamma_{0} h}{\gamma \mu} \sum_{i=1}^{2}\left(p_{i, h}^{n}-p_{i, h}^{n-1}, q_{i, h}\right)_{\Sigma}, \quad \gamma_{0}>0
$$

which controls the temporal interface pressure fluctuations induced by the fluid-solid splitting in time and, hence, avoids added-mass stability issues.

The next result establishes the conditional stability of the fully discrete method (18)-(19).

Theorem 2.3. Let $\left\{\left(\boldsymbol{u}_{h}^{n}, p_{h}^{n}, \dot{\boldsymbol{d}}_{h}^{n}, \boldsymbol{d}_{h}^{n}\right)\right\}_{n \geq 1}$ be the sequence given by 18)-19. Then, under conditions $\gamma \gtrsim C_{\mathrm{T}} / \tilde{c}_{\mathrm{s}}, \gamma \tau \lesssim h$ and $\gamma_{0} \gtrsim 1$, there holds

$$
E^{n} \lesssim E^{0}+\sum_{i=1}^{2}\left(\mu\left\|\boldsymbol{u}_{i, h}^{0}\right\|_{0, \Sigma}^{2}+\mu\left\|\boldsymbol{\epsilon}\left(\boldsymbol{u}_{i, h}^{0}\right)\right\|_{0, \Omega_{i, h}}^{2}+\frac{\gamma_{0} h}{\gamma \mu} \tau\left\|p_{i, h}^{0}\right\|_{0, \Sigma}^{2}\right)
$$

for $n \geq 1$.

Proof. The result follows by combining Lemma 2.1 with the arguments reported in [15, Section 5.1].

The main drawback of the scheme $\sqrt{18}-19$ is that it delivers poor accuracy in practice. More precisely, the explicit treatment of the penalty term in the solid sub-step (18), i.e,

$$
2 \frac{\gamma \mu}{h}\left(\dot{\boldsymbol{d}}_{h}^{n}-\left\{\left\{\boldsymbol{u}_{h}^{n-1}\right\}, \boldsymbol{w}_{h}\right)_{\Sigma}\right.
$$

induces an splitting error $\mathcal{O}(\tau / h)$ which is not uniform in $h$. In the spirit of [17, 18, we propose to circumvent this issue by combining (18)-19) with a predictor-corrector strategy (with $K \geq 0$ corrections). The resulting solution procedure is detailed in Algorithm 2, where we have used the notation

$$
\partial_{\tau} x^{n, k} \stackrel{\text { def }}{=} \frac{1}{\tau}\left(x^{n, k}-x^{n-1}\right) .
$$

The key idea is that if, instead of the first-order extrapolation 20 , we consider a second-order extrapolation of the fluid velocity $(r=2)$, after $K$ correction iterations, the error induced by the explicit treatment of the penalty term becomes

$$
\mathcal{O}\left(\left(\tau^{2} / h\right)^{K+1}\right)
$$

As a result, the contribution of the penalty term becomes $\mathcal{O}\left(\tau^{2} / h\right)$ with $K=0$ and, hence, $\tau=$ $\mathcal{O}(h)$ suffices to achieve overall first-order accuracy. Nevertheless, numerical evidence indicates that $K \geq 1$ is mandatory for stability (see, e.g., the discussion of Section 4.2.2).

Remark 2.8. The original stabilized explicit coupling scheme $\sqrt{18}-(19)$ can be retrieved from Algorithm 2 by taking $K=0$ (no correction) and with first-order extrapolation for the initial guess of the fluid velocity $(r=1)$. On the other hand, if we let $K \rightarrow \infty$ the splitting error (23) tends to zero and we retrieve the implicit coupling solution provided by Algorithm 1 . In other words, Algorithm 2 with enough correction iterations (i.e., until convergence) provides a partitioned iterative solution procedure for Algorithm 1 . 
Algorithm 2 Stabilized explicit coupling scheme with $K \geq 0$ corrections For $n \geq 1$ :

1. Set initial guess for predictor:

$$
\boldsymbol{u}_{h}^{n, 0}=\boldsymbol{u}_{h}^{n, \star}, \quad p_{h}^{n, 0}=p_{h}^{n-1}, \quad \boldsymbol{d}_{h}^{n, 0}=\boldsymbol{d}_{h}^{n-1}, \quad \dot{\boldsymbol{d}}_{h}^{n, 0}=\dot{\boldsymbol{d}}_{h}^{n-1} .
$$

2. Correction iterations. For $k=1, \ldots, K+1$ :

(a) Find $\left(\dot{\boldsymbol{d}}_{h}^{n, k}, \boldsymbol{d}_{h}^{n, k}\right) \in \boldsymbol{W}_{h} \times \boldsymbol{W}_{h}$ with $\dot{\boldsymbol{d}}_{h}^{n, k}=\partial_{\tau} \boldsymbol{d}_{h}^{n, k}$ and such that

$$
\begin{aligned}
\rho^{\mathrm{s}} \epsilon\left(\partial_{\tau} \dot{\boldsymbol{d}}_{h}^{n, k}, \boldsymbol{w}_{h}\right)_{\Sigma}+ & a^{\mathrm{s}}\left(\boldsymbol{d}_{h}^{n, k}, \boldsymbol{w}_{h}\right)+\frac{2 \gamma \mu}{h}\left(\dot{\boldsymbol{d}}_{h}^{n, k}, \boldsymbol{w}_{h}\right)_{\Sigma} \\
& =\frac{2 \gamma \mu}{h}\left(\left\{\left\{\boldsymbol{u}_{h}^{n, k-1}\right\}, \boldsymbol{w}_{h}\right)_{\Sigma}-\left(\llbracket \boldsymbol{\sigma}\left(\boldsymbol{u}_{h}^{n, k-1}, p_{h}^{n, k-1}\right) \boldsymbol{n} \rrbracket, \boldsymbol{w}_{h}\right)_{\Sigma}\right.
\end{aligned}
$$

for all $\boldsymbol{w}_{h} \in \boldsymbol{W}_{h}$.

(b) Find $\left(\boldsymbol{u}_{h}^{n, k}, p_{h}^{n, k}\right) \in \boldsymbol{V}_{h} \times Q_{h}$ such that

$$
\begin{array}{r}
\rho^{\mathrm{f}}\left(\partial_{\tau} \boldsymbol{u}_{h}^{n, k}, \boldsymbol{v}_{h}\right)_{\Omega}+a_{h}^{\mathrm{f}}\left(\left(\boldsymbol{u}_{h}^{n, k}, p_{h}^{n, k}\right),\left(\boldsymbol{v}_{h}, q_{h}\right)\right)+\frac{\gamma \mu}{h} \sum_{i=1}^{2}\left(\boldsymbol{u}_{i, h}^{n, k}, \boldsymbol{v}_{i, h}\right)_{\Sigma} \\
-\sum_{i=1}^{2}\left(\boldsymbol{u}_{i, h}^{n, k}-\dot{\boldsymbol{d}}_{h}^{n, k}, \boldsymbol{\sigma}\left(\boldsymbol{v}_{i, h},-q_{i, h}\right) \boldsymbol{n}_{i}\right)_{\Sigma}+\frac{\gamma_{0} h}{\gamma \mu} \sum_{i=1}^{2}\left(p_{i, h}^{n, k}-p_{i, h}^{n, k-1}, q_{i, h}\right)_{\Sigma} \\
=\frac{\gamma \mu}{h} \sum_{i=1}^{2}\left(\dot{\boldsymbol{d}}_{h}^{n, k}, \boldsymbol{v}_{i, h}\right)_{\Sigma}+\sum_{i=1}^{2}\left(\boldsymbol{\sigma}\left(\boldsymbol{u}_{i, h}^{n, k-1}, p_{i, h}^{n, k-1}\right) \boldsymbol{n}_{i}, \boldsymbol{v}_{i, h}\right)_{\Sigma}
\end{array}
$$

for all $\left(\boldsymbol{v}_{h}, q_{h}\right) \in \boldsymbol{V}_{h} \times Q_{h}$.

3. Set

$$
\boldsymbol{u}_{h}^{n}=\boldsymbol{u}_{h}^{n, K+1}, \quad p_{h}^{n}=p_{h}^{n, K+1}, \quad \boldsymbol{d}_{h}^{n}=\boldsymbol{d}_{h}^{n, K+1}, \quad \dot{\boldsymbol{d}}_{h}^{n}=\dot{\boldsymbol{d}}_{h}^{n, K+1} .
$$

\subsubsection{Semi-implicit schemes}

The main drawback of the explicit coupling schemes introduced in the previous section is that the splitting error is not uniform in $h$. Enough correction iterations with suitable predictions (Algorithm 2) are hence needed to enhance accuracy. In this section, we propose to overcome these issues through an operator splitting approach (see [31, Section 2]). The resulting schemes deliver stability and overall first-order accuracy (uniform in $h$ ) while keeping a certain degree of fluid-solid splitting (semi-implicit coupling schemes). The schemes herein presented extend the explicit Robin-Neumann paradigm introduced in [30, 32] to the case of immersed structures with unfitted meshes.

To this purpose, we consider the following fractional-step time-marching of (9): for $n>1$, 
1. Find $\left(\boldsymbol{u}_{h}^{n}, p_{h}^{n}, \dot{\boldsymbol{d}}_{h}^{n-\frac{1}{2}}\right) \in \boldsymbol{V}_{h} \times Q_{h} \times \boldsymbol{W}_{h}$ such that

$$
\begin{aligned}
& \rho^{\mathrm{f}}\left(\partial_{\tau} \boldsymbol{u}_{h}^{n}, \boldsymbol{v}_{h}\right)_{\Omega}+a_{h}^{\mathrm{f}}\left(\left(\boldsymbol{u}_{h}^{n}, p_{h}^{n}\right),\left(\boldsymbol{v}_{h}, q_{h}\right)\right)+\frac{\rho^{\mathrm{s}} \epsilon}{\tau}\left(\dot{\boldsymbol{d}}_{h}^{n-\frac{1}{2}}-\dot{\boldsymbol{d}}_{h}^{n-1}, \boldsymbol{w}_{h}\right)_{\Sigma} \\
& -\sum_{i=1}^{2}\left(\boldsymbol{\sigma}\left(\boldsymbol{u}_{i, h}^{n}, p_{i, h}^{n}\right) \boldsymbol{n}_{i}, \boldsymbol{v}_{i, h}-\boldsymbol{w}_{h}\right)_{\Sigma}-\sum_{i=1}^{2}\left(\boldsymbol{u}_{i, h}^{n}-\dot{\boldsymbol{d}}_{h}^{n-\frac{1}{2}}, \boldsymbol{\sigma}\left(\boldsymbol{v}_{i, h},-q_{i, h}\right) \boldsymbol{n}_{i}\right)_{\Sigma} \\
& +\sum_{i=1}^{2} \frac{\gamma \mu}{h}\left(\boldsymbol{u}_{i, h}^{n}-\dot{\boldsymbol{d}}_{h}^{n-\frac{1}{2}}, \boldsymbol{v}_{i, h}-\boldsymbol{w}_{h}\right)_{\Sigma}=-a^{\mathrm{s}}\left(\boldsymbol{d}_{h}^{n, \star}, \boldsymbol{w}_{h}\right)
\end{aligned}
$$

for all $\left(\boldsymbol{v}_{h}, q_{h}, \boldsymbol{w}_{h}\right) \in \boldsymbol{V}_{h} \times Q_{h} \times \boldsymbol{W}_{h}$.

2. Find $\left(\dot{\boldsymbol{d}}_{h}^{n}, \boldsymbol{d}_{h}^{n}\right) \in \boldsymbol{W}_{h} \times \boldsymbol{W}_{h}$ with $\dot{\boldsymbol{d}}_{h}^{n}=\partial_{\tau} \boldsymbol{d}_{h}^{n}$ and such that

$$
\frac{\rho^{\mathrm{s}} \epsilon}{\tau}\left(\dot{\boldsymbol{d}}_{h}^{n}-\dot{\boldsymbol{d}}_{h}^{n-\frac{1}{2}}, \boldsymbol{w}_{h}\right)_{\Sigma}+a^{\mathrm{s}}\left(\boldsymbol{d}_{h}^{n}, \boldsymbol{w}_{h}\right)=a^{\mathrm{s}}\left(\boldsymbol{d}_{h}^{n, \star}, \boldsymbol{w}_{h}\right)
$$

for all $\boldsymbol{w}_{h} \in \boldsymbol{W}_{h}$

The introduction in step (24) of the intermediate solid-velocity $\dot{\boldsymbol{d}}_{h}^{n-\frac{1}{2}}$ enables the implicit treatment of the solid inertia within the fluid. This is enough to guarantee added-mass free stability. The remaining solid elastic contributions are treated explicitly (or ignored) via extrapolation. The end-of-step velocity $\dot{\boldsymbol{d}}_{h}^{n} \in \boldsymbol{W}_{h}$ is recovered by solving the solid problem (25).

Remark 2.9. It should be noted that the intermediate solid-velocity $\dot{\boldsymbol{d}}_{h}^{n-\frac{1}{2}}$ cannot be eliminated in (24) and, hence, the coupling scheme is not explicit. This is a major difference with respect to the case of fitted meshes and conformal discretizations considered in [30, 32]. In that case, we can take $\dot{\boldsymbol{d}}_{h}^{n-\frac{1}{2}}=\left.\boldsymbol{u}_{h}^{n}\right|_{\Sigma}$ and $\boldsymbol{w}_{h}=\left.\boldsymbol{v}_{h}\right|_{\Sigma}$ in (24), which yields a standard fluid problem with an explicit Robin condition on the interface $\Sigma$.

Remark 2.10. The relation (24) has somme similarities with the explicit time-splitting procedures commonly used in the immersed boundary (IB) method (see, e.g., [58, 56. [7]). Indeed, sub-step (24) simultaneously includes the fluid and solid inertia whereas the solid elastic contributions are treated explicitly. The key difference concerns the solid sub-step (25), which in the IB method consists of a simple displacement-velocity relation (i.e., the structure solver is never called), which in practice enforces restrictive CFL conditions for stability. Theorem 2.4 below shows that 24 - 25] circumvents this issue.

It is worth noting that the semi-implicit coupling scheme provided by $(24)-25$ has a reduced computational complexity with respect to Algorithm 1. Indeed, the solid contribution to (24) reduces to a simple interface mass-matrix, which does not degrade the conditioning of the system matrix. This reduction in the coupling complexity is particularly important when considering general shell models (see, e.g., [22]), whose elastic contributions incorporate additional unknowns (e.g., rotations). Moreover, unlike Algorithm 2, the scheme [24 - 25 does not involve any correction iteration.

In the spirit of 32, the solid step 25 can be reformulated as a standard solid problem. Indeed, by taking $\boldsymbol{v}_{h}=\mathbf{0}$ and $q_{h}=0$ in $(24)$ and adding the resulting expression to (25), we get

$$
\begin{aligned}
\rho^{\mathrm{s}} \epsilon\left(\partial_{\tau} \dot{\boldsymbol{d}}_{h}^{n}, \boldsymbol{w}_{h}\right)_{\Sigma}+a^{\mathrm{s}}\left(\boldsymbol{d}_{h}^{n}, \boldsymbol{w}_{h}\right)= & -\left(\llbracket \boldsymbol{\sigma}\left(\boldsymbol{u}_{h}^{n}, p_{h}^{n}\right) \boldsymbol{n} \rrbracket, \boldsymbol{w}_{h}\right)_{\Sigma} \\
& +\frac{2 \gamma \mu}{h}\left(\left\{\boldsymbol{u}_{h}^{n}\right\}-\dot{\boldsymbol{d}}_{h}^{n-\frac{1}{2}}, \boldsymbol{w}_{h}\right)_{\Sigma}
\end{aligned}
$$

$\mathrm{RR} \mathrm{n}^{\circ} 8723$ 
for all $\boldsymbol{w}_{h} \in \boldsymbol{W}_{h}$. We can also avoid the extrapolations of the solid elastic term in 24) (which can be cumbersome in practice), by reformulating step (24) in a more intrinsic fashion. To this purpose, we note that from $(26)$, we have

$$
\begin{aligned}
a^{\mathrm{s}}\left(\boldsymbol{d}_{h}^{n, \star}, \boldsymbol{w}_{h}\right)= & -\rho^{\mathrm{s}} \epsilon\left(\partial_{\tau} \dot{\boldsymbol{d}}_{h}^{n, \star}, \boldsymbol{w}_{h}\right)_{\Sigma}-\left(\llbracket \boldsymbol{\sigma}\left(\boldsymbol{u}_{h}^{n, \star}, p_{h}^{n, \star}\right) \boldsymbol{n} \rrbracket, \boldsymbol{w}_{h}\right)_{\Sigma} \\
& +\frac{2 \gamma \mu}{h}\left(\left\{\boldsymbol{u}_{h}^{n, \star}\right\}-\dot{\boldsymbol{d}}_{h}^{n-\frac{1}{2}, \star}, \boldsymbol{w}_{h}\right)_{\Sigma}
\end{aligned}
$$

for all $\boldsymbol{w}_{h} \in \boldsymbol{W}_{h}$ and $n>r$. Owing to (26) and (27), the semi-implicit scheme (24)-(25) can be reformulated as shown in Algorithm 3 .

\section{Algorithm 3 Semi-implicit coupling scheme}

For $n>r$ :

1. Fluid-with-solid-inertia sub-step: find $\left(\boldsymbol{u}_{h}^{n}, p_{h}^{n}, \dot{\boldsymbol{d}}_{h}^{n-\frac{1}{2}}\right) \in \boldsymbol{V}_{h} \times Q_{h} \times \boldsymbol{W}_{h}$ such that

$$
\begin{aligned}
& \rho^{\mathrm{f}}\left(\partial_{\tau} \boldsymbol{u}_{h}^{n}, \boldsymbol{v}_{h}\right)_{\Omega}+a_{h}^{\mathrm{f}}\left(\left(\boldsymbol{u}_{h}^{n}, p_{h}^{n}\right),\left(\boldsymbol{v}_{h}, q_{h}\right)\right)+\frac{\rho^{\mathrm{s}} \epsilon}{\tau}\left(\dot{\boldsymbol{d}}_{h}^{n-\frac{1}{2}}, \boldsymbol{w}_{h}\right)_{\Sigma} \\
& -\sum_{i=1}^{2}\left(\boldsymbol{\sigma}\left(\boldsymbol{u}_{i, h}^{n}, p_{i, h}^{n}\right) \boldsymbol{n}_{i}, \boldsymbol{v}_{i, h}-\boldsymbol{w}_{h}\right)_{\Sigma}-\sum_{i=1}^{2}\left(\boldsymbol{u}_{i, h}^{n}-\dot{\boldsymbol{d}}_{h}^{n-\frac{1}{2}}, \boldsymbol{\sigma}\left(\boldsymbol{v}_{i, h},-q_{i, h}\right) \boldsymbol{n}_{i}\right)_{\Sigma} \\
& +\sum_{i=1}^{2} \frac{\gamma \mu}{h}\left(\boldsymbol{u}_{i, h}^{n}-\dot{\boldsymbol{d}}_{h}^{n-\frac{1}{2}}, \boldsymbol{v}_{i, h}-\boldsymbol{w}_{h}\right)_{\Sigma}=\frac{\rho^{\mathrm{s}} \epsilon}{\tau}\left(\dot{\boldsymbol{d}}_{h}^{n-1}+\tau \partial_{\tau} \dot{\boldsymbol{d}}_{h}^{n, \star}, \boldsymbol{w}_{h}\right)_{\Sigma} \\
& +\left(\llbracket \boldsymbol{\sigma}\left(\boldsymbol{u}_{h}^{n, \star}, p_{h}^{n, \star}\right) \boldsymbol{n} \rrbracket, \boldsymbol{w}_{h}\right)_{\Sigma}-\frac{2 \gamma \mu}{h}\left(\left\{\boldsymbol{u}_{h}^{n, \star}\right\}-\dot{\boldsymbol{d}}_{h}^{n-\frac{1}{2}, \star}, \boldsymbol{w}_{h}\right)_{\Sigma}
\end{aligned}
$$

for all $\left(\boldsymbol{v}_{h}, q_{h}, \boldsymbol{w}_{h}\right) \in \boldsymbol{V}_{h} \times Q_{h} \times \boldsymbol{W}_{h}$.

2. Solid sub-step: find $\left(\dot{\boldsymbol{d}}_{h}^{n}, \boldsymbol{d}_{h}^{n}\right) \in \boldsymbol{W}_{h} \times \boldsymbol{W}_{h}$ with $\dot{\boldsymbol{d}}_{h}^{n}=\partial_{\tau} \boldsymbol{d}_{h}^{n}$ and such that

$$
\begin{aligned}
\rho^{\mathrm{s}} \epsilon\left(\partial_{\tau} \dot{\boldsymbol{d}}_{h}^{n}, \boldsymbol{w}_{h}\right)_{\Sigma}+a^{\mathrm{s}}\left(\boldsymbol{d}_{h}^{n}, \boldsymbol{w}_{h}\right)= & -\left(\llbracket \boldsymbol{\sigma}\left(\boldsymbol{u}_{h}^{n}, p_{h}^{n}\right) \boldsymbol{n} \rrbracket, \boldsymbol{w}_{h}\right)_{\Sigma} \\
& +\frac{2 \gamma \mu}{h}\left(\left\{\left\{\boldsymbol{u}_{h}^{n}\right\}-\dot{\boldsymbol{d}}_{h}^{n-\frac{1}{2}}, \boldsymbol{w}_{h}\right)_{\Sigma}\right.
\end{aligned}
$$

for all $\boldsymbol{w}_{h} \in \boldsymbol{W}_{h}$.

Remark 2.11. Algorithm 3 with $r=1,2$ is a multi-step method on the interface. Thus, additional data is needed to start the time-marching. In practice, this data can be obtained by performing one step of the scheme with $r=0$ and then one step of the scheme with $r=1$.

The following result states the stability and convergence properties of the semi-implicit schemes reported in Algorithm 3 .

Theorem 2.4. Let $\left\{\left(\boldsymbol{u}_{h}^{n}, p_{h}^{n}, \dot{\boldsymbol{d}}_{h}^{n-\frac{1}{2}}, \boldsymbol{d}_{h}^{n}, \dot{\boldsymbol{d}}_{h}^{n}\right)\right\}_{n>r}$ be the sequence given by Algorithm 3 , initialized as indicated in Remark 2.11 for $r \geq 1$. Let $\gamma>0$ be given by Lemma 2.1. There holds

$$
E^{n} \leq E^{0}
$$

for all $n>r$, unconditionally for $r \in\{0,1\}$ and under the CFL-like condition $\tau \lesssim \alpha h^{\frac{6}{5}}, \tau \alpha<1$ and $\alpha>0$, for $r=2$. In addition, for smooth enough solutions, there holds

$$
\mathcal{E}^{n} \lesssim h+\tau+\tau^{2^{r-1}}
$$


Proof. The result follows from a straightforward adaptation of the arguments used in [31, Proposition 2.1].

The contribution of the splitting error in the a priori energy estimate of Theorem 2.4 is given by the $\mathcal{O}\left(\tau^{2^{r-1}}\right)$ term. Note that this guarantees the $h$-uniformity of the error, which is a major advantage with respect to Algorithm 2. Moreover, Algorithm 3 with $r=1$ simultaneously yields unconditional stability and overall first-order accuracy, without resorting to any correction iteration.

Remark 2.12. Algorithms 1 [3 can be adapted to the time discretisation of (14) with minor modifications. Indeed, it suffices to add the corresponding fictitious fluid-fluid interface terms

$$
-\left(\left\{\boldsymbol{\sigma}\left(\boldsymbol{u}_{h}^{n}, p_{h}^{n}\right)\right\} \boldsymbol{n}, \llbracket \boldsymbol{v}_{h} \rrbracket\right)_{\widetilde{\Sigma}_{h}}-\left(\left\{\left[\boldsymbol{\sigma}\left(\boldsymbol{v}_{h},-q_{h}\right)\right\} \boldsymbol{n}, \llbracket \boldsymbol{u}_{h}^{n} \rrbracket\right)_{\widetilde{\Sigma}_{h}}+\frac{\gamma \mu}{h}\left(\llbracket \boldsymbol{u}_{h}^{n} \rrbracket, \llbracket \boldsymbol{v}_{h} \rrbracket\right)_{\widetilde{\Sigma}_{h}}\right.
$$

to the discrete problems (15), 22) and (28), respectively.

\section{The non-linear case: dynamic interfaces}

In this section we extend the numerical methods of Section 2.2 to the case of a non-linear fluid-structure interaction problem, involving an incompressible viscous fluid and an immersed thin-walled structure. The fluid is described by the incompressible Navier-Stokes equations and the structure by a (possibly) non-linear membrane or shell model.

\subsection{Problem setting}

Let $\Sigma \subset \mathbb{R}^{d}$ be the reference configuration of the solid mid-surface. The current position of the interface, denoted by $\Sigma(t)$, is parametrized by the one-to-one deformation map $\phi: \Sigma \times \mathbb{R}^{+} \longrightarrow \mathbb{R}^{d}$ as $\Sigma(t)=\boldsymbol{\phi}(\Sigma, t)$, with $\phi \stackrel{\text { def }}{=} \boldsymbol{I}_{\Sigma \times \mathbb{R}^{+}}+\boldsymbol{d}$ and where $\boldsymbol{d}$ denotes the displacement of the solid. In order to ease the presentation, we introduce the notation $\phi_{t} \stackrel{\text { def }}{=} \phi(\cdot, t)$, so that we also have $\Sigma(t)=\phi_{t}(\Sigma)$. The structure is supposed to move within a domain $\Omega \subset \mathbb{R}^{d}$ with boundary $\Gamma \stackrel{\text { def }}{=} \partial \Omega$ (see Figure 7). For simplicity and without loss of generality, $\Omega$ is assumed to be fixed. The fluid is described in the time-dependent control volume

$$
\Omega(t) \stackrel{\text { def }}{=} \Omega \backslash \Sigma(t) \subset \mathbb{R}^{d}
$$

with its boundary partitioned as $\partial \Omega(t)=\Sigma(t) \cup \Gamma$.

The considered non-linear coupled problem reads as follow: find the fluid velocity and pressure $\boldsymbol{u}: \Omega \times \mathbb{R}^{+} \rightarrow \mathbb{R}^{d}, p: \Omega \times \mathbb{R}^{+} \rightarrow \mathbb{R}$ and the solid displacement and velocity $\boldsymbol{d}: \Sigma \times \mathbb{R}^{+} \rightarrow \mathbb{R}^{d}$, $\dot{d}: \Sigma \times \mathbb{R}^{+} \rightarrow \mathbb{R}^{d}$ such that

$$
\begin{aligned}
& \left\{\begin{array}{r}
\Omega(t) \stackrel{\text { def }}{=} \Omega \backslash \Sigma(t), \quad \Sigma(t)=\phi_{t}(\Sigma), \quad \phi_{t} \stackrel{\text { def }}{=} \boldsymbol{I}_{\Sigma}+\boldsymbol{d}(t), \\
\rho^{\mathrm{f}}\left(\partial_{t} \boldsymbol{u}+(\boldsymbol{u} \cdot \nabla) \boldsymbol{u}\right)-\operatorname{div} \boldsymbol{\sigma}(\boldsymbol{u}, p)=\mathbf{0} \quad \text { in } \Omega(t), \\
\operatorname{div} \boldsymbol{u}=0 \quad \text { in } \Omega(t), \\
\boldsymbol{u}=\mathbf{0} \quad \text { on } \quad \Gamma,
\end{array}\right. \\
& \left\{\begin{array}{l}
\boldsymbol{u}_{1}=\boldsymbol{u}_{2}=\dot{\boldsymbol{d}} \circ \boldsymbol{\phi}_{t}^{-1} \quad \text { on } \quad \Sigma(t), \\
\boldsymbol{d}(t) \in \boldsymbol{W}, \quad \dot{\boldsymbol{d}}=\partial_{t} \boldsymbol{d}, \\
\rho^{\mathrm{s}} \epsilon\left(\partial_{t} \dot{\boldsymbol{d}}, \boldsymbol{w}\right)_{\Sigma}+a^{\mathrm{s}}(\boldsymbol{d}, \boldsymbol{w})=-\int_{\Sigma(t)} \llbracket \boldsymbol{\sigma}(\boldsymbol{u}, p) \boldsymbol{n} \rrbracket \cdot \boldsymbol{w} \circ \boldsymbol{\phi}_{t}^{-1} \quad \forall \boldsymbol{w} \in \boldsymbol{W},
\end{array}\right.
\end{aligned}
$$




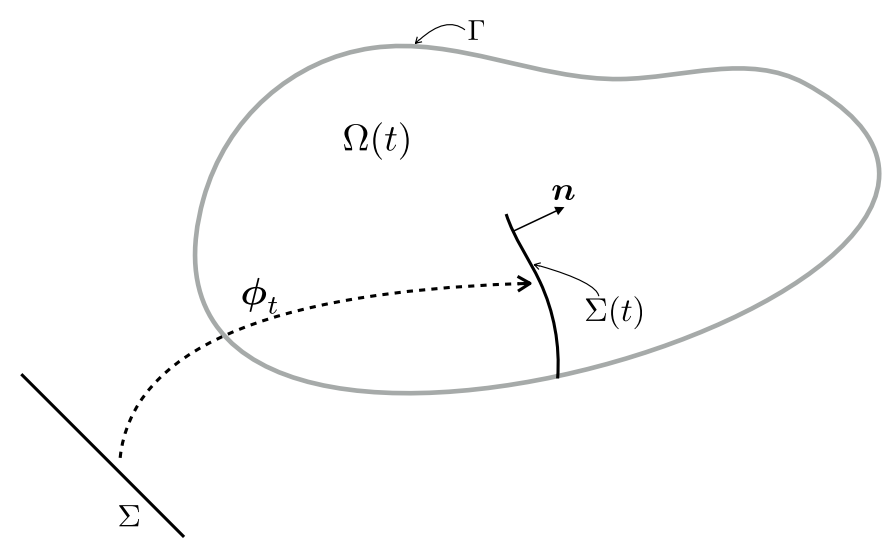

Figure 7: Geometric description.

complemented with standard initial conditions $\boldsymbol{u}(0)=\boldsymbol{u}_{0}, \boldsymbol{d}(0)=\boldsymbol{d}_{0}$ and $\dot{\boldsymbol{d}}(0)=\dot{\boldsymbol{d}}_{0}$. We recall that $\boldsymbol{W} \subset\left[H^{1}(\Sigma)\right]^{d}$ denotes the space of solid admissible displacements and $a^{\mathrm{s}}: \boldsymbol{W} \times \boldsymbol{W} \rightarrow \mathbb{R}$ describes the (possibly non-linear) elastic behavior of the structure.

Remark 3.1. The mixed Eulerian-Lagrangian nature of the problem $(29)-(30)$ is emphasized by the presence of the map $\phi_{t}^{-1}$, which transforms the solid quantities from the reference to the current configuration. This introduces geometrical non-linearities.

For the fluid, we consider the same velocity and pressure functional spaces as in Section 2 and we introduce the convective tri-linear form

$$
c_{\Omega}(\boldsymbol{z}, \boldsymbol{u}, \boldsymbol{v}) \stackrel{\text { def }}{=} \rho^{\mathrm{f}}(\boldsymbol{z} \cdot \boldsymbol{\nabla} \boldsymbol{u}, \boldsymbol{v})_{\Omega} .
$$

We recall that, if $\boldsymbol{z} \in\{\boldsymbol{v} \in \boldsymbol{V} / \operatorname{div} \boldsymbol{v}=0$ in $\Omega\}$, using integration by parts we have

$$
c_{\Omega}(\boldsymbol{z}, \boldsymbol{v}, \boldsymbol{v})=0 \quad \forall \boldsymbol{v} \in \boldsymbol{V} .
$$

The weak form of the linear coupled problem $29-(30)$ reads as follows: for $t>0$, find $(\boldsymbol{u}(t), p(t), \boldsymbol{d}(t), \dot{\boldsymbol{d}}(t)) \in \boldsymbol{V} \times Q \times \boldsymbol{W} \times \boldsymbol{W}$ such that

$$
\left\{\begin{array}{l}
\phi_{t}=\boldsymbol{I}_{\Sigma}+\boldsymbol{d}(t), \quad \Sigma(t)=\phi_{t}(\Sigma), \\
\boldsymbol{u}_{1}=\boldsymbol{u}_{2}=\dot{\boldsymbol{d}} \circ \phi_{t}^{-1} \quad \text { on } \quad \Sigma(t), \quad \dot{\boldsymbol{d}}=\partial_{t} \boldsymbol{d}, \\
\rho^{\mathrm{f}}\left(\partial_{t} \boldsymbol{u}, \boldsymbol{v}\right)_{\Omega}+c_{\Omega}(\boldsymbol{u}, \boldsymbol{u}, \boldsymbol{v})+a_{\Omega}^{\mathrm{f}}((\boldsymbol{u}, p),(\boldsymbol{v}, q)) \\
+\rho^{\mathrm{s}} \epsilon\left(\partial_{t} \dot{\boldsymbol{d}}, \boldsymbol{w}\right)_{\Sigma}+a^{\mathrm{s}}(\boldsymbol{d}, \boldsymbol{w})=0
\end{array}\right.
$$

for all $(\boldsymbol{v}, q, \boldsymbol{w}) \in \boldsymbol{V} \times Q \times \boldsymbol{W}$ with $\left.\boldsymbol{v}\right|_{\Sigma(t)}=\boldsymbol{w} \circ \boldsymbol{\phi}_{t}^{-1}$.

Assuming that $a^{\mathrm{s}}\left(\boldsymbol{d}, \partial_{t} \boldsymbol{d}\right)=\frac{1}{2} \partial_{t} a^{\mathrm{s}}(\boldsymbol{d}, \boldsymbol{d})$, taking $(\boldsymbol{v}, q, \boldsymbol{w})=(\boldsymbol{u}(t), p(t), \dot{\boldsymbol{d}}(t))$ in 33 and using (32) we retrieve an energy equality similar to (4).

\subsection{Numerical methods}

The next section presents the formulation of the numerical methods introduced in Section 2.2 within the framework of the non-linear coupled problem $(29)-30$. The basic idea consists in combining the different coupling paradigms with an explicit treatment of the geometrical compatibility 29$]_{1}$. Finally, in Section 3.2 .2 , we briefly comment on how to handle the integration of quantities associated with different time levels. 


\subsubsection{Nitsche-XFEM formulation and coupling schemes}

For simplicity, we assume that $\Omega$ and $\Sigma$ are polyhedral. We consider the general case in which the interface partially intersects the fluid domain (see Figure 7). The approximation space for the solid, $\boldsymbol{W}_{h}$, is the same as in Section 2.1. For a given discrete displacement $\boldsymbol{d}_{h}^{n-1} \in \boldsymbol{W}_{h}$ at time level $n-1$, we introduce its corresponding deformation map $\boldsymbol{\phi}_{h}^{n-1} \stackrel{\text { def }}{=} \boldsymbol{I}_{\Sigma}+\boldsymbol{d}_{h}^{n-1}$. The current configuration (i.e., at time level $n$ ) of the discrete interface is defined as

$$
\Sigma_{h}^{n} \stackrel{\text { def }}{=} \phi_{h}^{n-1}(\Sigma)
$$

In other words, the geometric compatibility $(29)_{1}$ is treated in an explicit fashion.

For the construction of the fluid discrete spaces, we proceed as in Section 2.1.3 with the sole difference that, in the present framework, the fluid-solid interface $\Sigma_{h}^{n}$ depends on the discrete displacement $\boldsymbol{d}_{h}^{n-1}$. As a result, the fictitious fluid-fluid interface $\widetilde{\Sigma}_{h}^{n} \cup \widehat{\Sigma}_{h}^{n}$ and the fluid spaces, $\boldsymbol{V}_{h}^{n}$ and $Q_{h}^{n}$ given by $(13)$, depend both on the mesh step $h$ and on the time level $n$. We recall that the functions of these spaces are continuous in the fluid domain

$$
\Omega_{h}^{n} \stackrel{\text { def }}{=} \Omega \backslash\left(\Sigma_{h}^{n} \cup \widetilde{\Sigma}_{h}^{n}\right),
$$

but discontinuous across the moving interface $\Sigma_{h}^{n} \cup \widetilde{\Sigma}_{h}^{n}$.

It should be noted that the discrete fluid velocities do not satisfy the assumptions of (32), namely, they are not divergence free and $\boldsymbol{V}_{h}^{n} \not \subset \boldsymbol{V}$. Therefore, we need to modify the trilinear form (31) in order to retrieve a suitable discrete counterpart of $(32)$. This is a well-known issue when dealing with discontinuous Galerkin approximations of the Navier-Stokes equations (see, e.g., [25, Section 6.2.2]). The key idea consists in combining the so-called Temam's trick with the fact that, owing to $301_{1}$, the velocity field $\boldsymbol{u}$ is continuous across $\Sigma_{h}^{n} \cup \widetilde{\Sigma}_{h}^{n}$. To this purpose, we introduce the discrete tri-linear form

$$
\begin{aligned}
c_{h}^{n}\left(\boldsymbol{z}_{h}, \boldsymbol{u}_{h}, \boldsymbol{v}_{h}\right) \stackrel{\text { def }}{=} c_{\Omega_{h}^{n}}\left(\boldsymbol{z}_{h}, \boldsymbol{u}_{h}, \boldsymbol{v}_{h}\right)+\frac{\rho^{\mathrm{f}}}{2}\left(\left(\operatorname{div} \boldsymbol{z}_{h}\right) \boldsymbol{u}_{h}, \boldsymbol{v}_{h}\right)_{\Omega_{h}^{n}} \\
-\rho^{\mathrm{f}}\left(\left\{\left\{\boldsymbol{z}_{h} \rrbracket \cdot \boldsymbol{n} \llbracket \boldsymbol{u}_{h} \rrbracket,\left\{\left\{\boldsymbol{v}_{h}\right\}\right)_{\Sigma_{h}^{n} \cup \widetilde{\Sigma}_{h}^{n}}-\frac{\rho^{\mathrm{f}}}{2}\left(\llbracket \boldsymbol{z}_{h} \cdot \boldsymbol{n} \rrbracket,\left\{\boldsymbol{u}_{h} \cdot \boldsymbol{v}_{h}\right\}\right)_{\Sigma_{h}^{n} \cup \widetilde{\Sigma}_{h}^{n}} .\right.\right.\right.
\end{aligned}
$$

Note that the last three terms are strongly consistent. Moreover, using integration by parts in $\Omega_{h}^{n}$ we can infer that, if $\boldsymbol{z}_{h} \in \boldsymbol{V}_{h}^{n}$, there holds

$$
c_{h}^{n}\left(\boldsymbol{z}_{h}, \boldsymbol{v}_{h}, \boldsymbol{v}_{h}\right)=0 \quad \forall \boldsymbol{v}_{h} \in \boldsymbol{V}_{h}^{n} .
$$

To cope with the numerical instabilities related to the inf-sup incompatibility of the discrete spaces and to large local Reynolds numbers, we need to resort to a stabilisation method (see, e.g., [11, 46, 69, 23, 19, 9, 14, 36, 3] and the references therein). The objective of such a procedure is basically twofold: guarantee the well-posedness of the discrete problem and improve the convergence of the approximations while limiting the propagation of spurious oscillations. A successful approach is the so-called SUPG/PSPG method (see, e.g., [1, 46, 69]), which offers an unified treatment of the velocity and pressure stabilization by adding to the Galerkin formulation an element-wise weighted residual of the equation. The residual based nature of the stabilization operator guarantees the consistency of the method.

Within the present unfitted framework this last property is more delicate. Indeed, as discussed in Section 2.1.1 (see also [21, 18]), the theoretical analysis indicates that the stabilization operator must act on the whole computational domain, that is, including the fictitious zone of 
Algorithm 4 Non-linear version of Algorithm 1 .

For $n \geq 1$,

1. Interface update: $\Omega_{h}^{n}=\Omega \backslash\left(\Sigma_{h}^{n} \cup \widetilde{\Sigma}_{h}^{n}\right), \Sigma_{h}^{n}=\boldsymbol{\phi}_{h}^{n-1}(\Sigma), \boldsymbol{\phi}_{h}^{n-1}=\boldsymbol{I}_{\Sigma}+\boldsymbol{d}_{h}^{n-1}$.

2. Find $\left(\boldsymbol{u}_{h}^{n}, p_{h}^{n}, \dot{\boldsymbol{d}}_{h}^{n}, \boldsymbol{d}_{h}^{n}\right) \in \boldsymbol{V}_{h}^{n} \times Q_{h}^{n} \times \boldsymbol{W}_{h} \times \boldsymbol{W}_{h}$, such that $\dot{\boldsymbol{d}}_{h}=\partial_{\tau} \boldsymbol{d}_{h}^{n}$ and

$$
\left\{\begin{array}{l}
\rho^{\mathrm{f}}\left(\partial_{\tau} \boldsymbol{u}_{h}^{n}, \boldsymbol{v}_{h}\right)_{\Omega}+a_{h}^{\mathrm{f}, n}\left(\boldsymbol{u}_{h}^{n} ;\left(\boldsymbol{u}_{h}^{n}, p_{h}^{n}\right),\left(\boldsymbol{v}_{h}, q_{h}\right)\right)+\rho^{\mathrm{s}} \epsilon\left(\partial_{\tau} \dot{\boldsymbol{d}}_{h}^{n}, \boldsymbol{w}_{h}\right)_{\Sigma}+a^{\mathrm{s}}\left(\boldsymbol{d}_{h}^{n}, \boldsymbol{w}_{h}\right) \\
-\sum_{i=1}^{2}\left(\boldsymbol{\sigma}\left(\boldsymbol{u}_{i, h}^{n}, p_{i, h}^{n}\right) \boldsymbol{n}_{i}, \boldsymbol{v}_{i, h}-\boldsymbol{w}_{h}\right)_{\Sigma_{h}^{n}}-\sum_{i=1}^{2}\left(\boldsymbol{u}_{i, h}^{n}-\dot{\boldsymbol{d}}_{h}^{n}, \boldsymbol{\sigma}\left(\boldsymbol{v}_{i, h},-q_{i, h}\right) \boldsymbol{n}_{i}\right)_{\Sigma_{h}^{n}} \\
-\left(\left\{\boldsymbol{\sigma}\left(\boldsymbol{u}_{h}^{n}, p_{h}^{n}\right)\right\} \boldsymbol{n}, \llbracket \boldsymbol{v}_{h} \rrbracket\right)_{\widetilde{\Sigma}_{h}^{n}}-\left(\left\{\left\{\boldsymbol{\sigma}\left(\boldsymbol{v}_{h},-q_{h}\right)\right\} \boldsymbol{n}, \llbracket \boldsymbol{u}_{h}^{n} \rrbracket\right)_{\widetilde{\Sigma}_{h}^{n}}\right. \\
+\frac{\gamma \mu}{h} \sum_{i=1}^{2}\left(\boldsymbol{u}_{i, h}^{n}-\dot{\boldsymbol{d}}_{h}^{n}, \boldsymbol{v}_{i, h}-\boldsymbol{w}_{h}\right)_{\Sigma_{h}^{n}}+\frac{\gamma \mu}{h}\left(\llbracket \boldsymbol{u}_{h}^{n} \rrbracket, \llbracket \boldsymbol{v}_{h} \rrbracket\right)_{\widetilde{\Sigma}_{h}^{n}}=0
\end{array}\right.
$$

for all $\left(\boldsymbol{v}_{h}, q_{h}, \boldsymbol{w}_{h}\right) \in \boldsymbol{V}_{h}^{n} \times Q_{h}^{n} \times \boldsymbol{W}_{h}$.

the overlapping region. However, in this zone, we cannot guarantee that the residual of a smooth extension of the solution vanishes. An alternative to circumvent this issue (see also [63]) is to use symmetric stabilization methods whose consistency does not rely on the residual (see, e.g., [23, 19, 14, 36, 3]). As an example, we consider here the continuous interior penalty (CIP) stabilization method of [19, 14]. To this purpose, we first introduce the set $\mathcal{F}_{i, h}^{n}$ of interior edges or faces of $\mathcal{T}_{i, h}^{n, \mathrm{f}}$. The corresponding velocity and pressure stabilization operators are, respectively, given by the relations

$$
\begin{aligned}
s_{\mathrm{v}, h}^{n}\left(\boldsymbol{z}_{h} ; \boldsymbol{u}_{h}, \boldsymbol{v}_{h}\right) \stackrel{\text { def }}{=} \gamma_{\mathrm{v}, 1} h^{2} \sum_{i=1}^{2} \sum_{F \in \mathcal{F}_{i, h}^{n}} \xi\left(\operatorname{Re}_{F}\left(\boldsymbol{z}_{h}\right)\right)\left\|\boldsymbol{z}_{h} \cdot \boldsymbol{n}\right\|_{L^{\infty}(F)}\left(\llbracket \nabla \boldsymbol{u}_{h} \rrbracket_{F}, \llbracket \boldsymbol{\nabla} \boldsymbol{v}_{h} \rrbracket_{F}\right)_{F} \\
+\gamma_{\mathrm{v}, 2} h^{2} \sum_{i=1}^{2} \sum_{F \in \mathcal{F}_{i, h}^{n}}\left\|\boldsymbol{z}_{h}\right\|_{L^{\infty}(F)}\left(\llbracket \operatorname{div} \boldsymbol{u}_{h} \rrbracket_{F}, \llbracket \operatorname{div} \boldsymbol{v}_{h} \rrbracket_{F}\right)_{F}, \\
s_{\mathrm{p}, h}^{n}\left(\boldsymbol{z}_{h} ; p_{h}, q_{h}\right) \stackrel{\text { def }}{=} \gamma_{\mathrm{p}} h^{2} \sum_{i=1}^{2} \sum_{F \in \mathcal{F}_{i, h}^{n}} \frac{\xi\left(\operatorname{Re}_{F}\left(\boldsymbol{z}_{h}\right)\right)}{\left\|\boldsymbol{z}_{h}\right\|_{L^{\infty}(F)}}\left(\llbracket \boldsymbol{\nabla} p_{h} \rrbracket_{F}, \llbracket \nabla q_{h} \rrbracket_{F}\right)_{F},
\end{aligned}
$$

where $\operatorname{Re}_{F}\left(\boldsymbol{z}_{h}\right) \stackrel{\text { def }}{=} \rho^{\mathrm{f}}\left\|\boldsymbol{z}_{h}\right\|_{L^{\infty}(F)} h \mu^{-1}$ denotes the local Reynolds number, $\xi(x) \stackrel{\text { def }}{=} \min \{1, x\}$ is a cut-off function and $\gamma_{\mathrm{p}}, \gamma_{\mathrm{v}, i}>0, i=1,2$, are user-defined parameters. At last, we collect all the above fluid contributions in a single term

$$
\begin{aligned}
a_{h}^{\mathrm{f}, n}\left(\boldsymbol{z}_{h} ;\left(\boldsymbol{u}_{h}, p_{h}\right),\left(\boldsymbol{v}_{h}, q_{h}\right)\right) \stackrel{\text { def }}{=} & c_{h}^{n}\left(\boldsymbol{z}_{h}, \boldsymbol{u}_{h}, \boldsymbol{v}_{h}\right)+a_{\Omega_{h}^{n}}^{\mathrm{f}}\left(\left(\boldsymbol{u}_{h}, p_{h}\right),\left(\boldsymbol{v}_{h}, q_{h}\right)\right) \\
& +s_{\mathrm{v}, h}^{n}\left(\boldsymbol{z}_{h} ; \boldsymbol{u}_{h}, \boldsymbol{v}_{h}\right)+s_{\mathrm{p}, h}^{n}\left(\boldsymbol{z}_{h} ; p_{h}, q_{h}\right)+g_{h}^{n}\left(\boldsymbol{u}_{h}, \boldsymbol{v}_{h}\right),
\end{aligned}
$$

with the time-dependent ghost-penalty operator now given by

$$
g_{h}^{n}\left(\boldsymbol{u}_{h}, \boldsymbol{v}_{h}\right) \stackrel{\text { def }}{=} \gamma_{\mathrm{g}} \mu h \sum_{i=1}^{2} \sum_{F \in \mathcal{F}_{i, h}^{n, \Sigma}}\left(\llbracket \nabla \boldsymbol{u}_{i, h} \rrbracket_{F}, \llbracket \nabla \boldsymbol{v}_{i, h} \rrbracket_{F}\right)_{F}
$$


Algorithm 5 Non-linear version of Algorithm 2

For $n \geq 1$ :

1. Interface update: $\boldsymbol{\phi}_{h}^{n-1}=\boldsymbol{I}_{\Sigma}+\boldsymbol{d}_{h}^{n-1}, \Sigma_{h}^{n}=\boldsymbol{\phi}_{h}^{n-1}(\Sigma), \Omega_{h}^{n}=\Omega \backslash\left(\Sigma_{h}^{n} \cup \widetilde{\Sigma}_{h}^{n}\right)$.

2. Set initial guess for predictor:

$$
\boldsymbol{u}_{h}^{n, 0}=\boldsymbol{u}_{h}^{n, \star}, \quad p_{h}^{n, 0}=p_{h}^{n-1}, \quad \boldsymbol{d}_{h}^{n, 0}=\boldsymbol{d}_{h}^{n-1}, \quad \dot{\boldsymbol{d}}_{h}^{n, 0}=\dot{\boldsymbol{d}}_{h}^{n-1} .
$$

3. Correction iterations. For $k=1, \ldots, K+1$ :

(a) Find $\left(\dot{\boldsymbol{d}}_{h}^{n, k}, \boldsymbol{d}_{h}^{n, k}\right) \in \boldsymbol{W}_{h} \times \boldsymbol{W}_{h}$ with $\dot{\boldsymbol{d}}_{h}^{n, k}=\partial_{\tau} \boldsymbol{d}_{h}^{n, k}$ and such that

$$
\begin{aligned}
\rho^{\mathrm{s}} \epsilon\left(\partial_{\tau} \dot{\boldsymbol{d}}_{h}^{n, k}, \boldsymbol{w}_{h}\right)_{\Sigma}+a^{\mathrm{s}} & \left.\boldsymbol{d}_{h}^{n, k}, \boldsymbol{w}_{h}\right)+\frac{2 \gamma \mu}{h}\left(\dot{\boldsymbol{d}}_{h}^{n, k}, \boldsymbol{w}_{h}\right)_{\Sigma_{h}^{n}} \\
= & \frac{2 \gamma \mu}{h}\left(\left\{\boldsymbol{u}_{h}^{n, k-1} \rrbracket, \boldsymbol{w}_{h}\right)_{\Sigma_{h}^{n}}-\left(\llbracket \boldsymbol{\sigma}\left(\boldsymbol{u}_{h}^{n, k-1}, p_{h}^{n, k-1}\right) \boldsymbol{n} \rrbracket, \boldsymbol{w}_{h}\right)_{\Sigma_{h}^{n}}\right.
\end{aligned}
$$

for all $\boldsymbol{w}_{h} \in \boldsymbol{W}_{h}$.

(b) Find $\left(\boldsymbol{u}_{h}^{n, k}, p_{h}^{n, k}\right) \in \boldsymbol{V}_{h}^{n} \times Q_{h}^{n}$ such that

$$
\begin{gathered}
\rho^{\mathrm{f}}\left(\partial_{\tau} \boldsymbol{u}_{h}^{n, k}, \boldsymbol{v}_{h}\right)_{\Omega}+a_{h}^{\mathrm{f}, n}\left(\boldsymbol{u}_{h}^{n, k} ;\left(\boldsymbol{u}_{h}^{n, k}, p_{h}^{n, k}\right),\left(\boldsymbol{v}_{h}, q_{h}\right)\right)+\frac{\gamma \mu}{h} \sum_{i=1}^{2}\left(\boldsymbol{u}_{i, h}^{n, k}, \boldsymbol{v}_{i, h}\right)_{\Sigma_{h}^{n}} \\
-\sum_{i=1}^{2}\left(\boldsymbol{u}_{i, h}^{n, k}-\dot{\boldsymbol{d}}_{h}^{n, k}, \boldsymbol{\sigma}\left(\boldsymbol{v}_{i, h},-q_{i, h}\right) \boldsymbol{n}_{i}\right)_{\Sigma_{h}^{n}}+\frac{\gamma_{0} h}{\gamma \mu} \sum_{i=1}^{2}\left(p_{i, h}^{n, k}-p_{i, h}^{n, k-1}, q_{i, h}\right)_{\Sigma_{h}^{n}} \\
-\left(\left\{\left\{\boldsymbol{\sigma}\left(\boldsymbol{u}_{h}^{n, k}, p_{h}^{n, k}\right)\right\} \boldsymbol{n}, \llbracket \boldsymbol{v}_{h} \rrbracket\right)_{\widetilde{\Sigma}_{h}^{n}}-\left(\left\{\left\{\boldsymbol{\sigma}\left(\boldsymbol{v}_{h},-q_{h}\right)\right\} \boldsymbol{n}, \llbracket \boldsymbol{u}_{h}^{n, k} \rrbracket\right)_{\widetilde{\Sigma}_{h}^{n}}\right.\right. \\
+\frac{\gamma \mu}{h}\left(\llbracket \boldsymbol{u}_{h}^{n, k} \rrbracket, \llbracket \boldsymbol{v}_{h} \rrbracket\right)_{\widetilde{\Sigma}_{h}^{n}}=\frac{\gamma \mu}{h} \sum_{i=1}^{2}\left(\dot{\boldsymbol{d}}_{h}^{n, k}, \boldsymbol{v}_{i, h}\right)_{\Sigma_{h}^{n}}+\sum_{i=1}^{2}\left(\boldsymbol{\sigma}\left(\boldsymbol{u}_{i, h}^{n, k-1}, p_{i, h}^{n, k-1}\right) \boldsymbol{n}_{i}, \boldsymbol{v}_{i, h}\right)_{\Sigma_{h}^{n}}
\end{gathered}
$$

for all $\left(\boldsymbol{v}_{h}, q_{h}\right) \in \boldsymbol{V}_{h}^{n} \times Q_{h}^{n}$.

4. Set $\boldsymbol{u}_{h}^{n}=\boldsymbol{u}_{h}^{n, K+1}, \quad p_{h}^{n}=p_{h}^{n, K+1}, \quad \boldsymbol{d}_{h}^{n}=\boldsymbol{d}_{h}^{n, K+1}, \quad \dot{\boldsymbol{d}}_{h}^{n}=\dot{\boldsymbol{d}}_{h}^{n, K+1}$.

and where $\mathcal{F}_{i, h}^{n, \Sigma}$ denotes the set of interior edges or faces of the elements intersected by $\Sigma_{h}^{n}$.

We now have all the ingredients to extend the numerical methods of Section 2.2 to the approximation of the non-linear coupled problem $(29)-(30)$. By combining the explicit treatment of the geometric compatibility (34) with the strongly coupled paradigm of Section 2.2.1 we get the solution procedure given in Algorithm 4 .

Remark 3.2. Note that all the appearances of $\dot{\boldsymbol{d}}_{h}^{n}$ and $\boldsymbol{w}_{h}$ in the interface terms of $\Sigma_{h}^{n}$ must be understood as $\dot{\boldsymbol{d}}_{h}^{n} \circ\left(\phi_{h}^{n}\right)^{-1}$ and $\boldsymbol{w}_{h} \circ\left(\phi_{h}^{n}\right)^{-1}$, respectively. The purpose of this little abuse of notation is simply to ease the presentation.

In Algorithm 5 we have reported the non-linear counterpart of the stabilized explicit coupling paradigm of Section 2.2.2 It should be noted that the $K \geq 0$ corrections are performed with the same configuration of the interface $\Sigma_{h}^{n}$. At last, Algorithm 6 presents the extension of the semi-implicit scheme introduced in Section 2.2.3 to the present non-linear framework. 
\begin{tabular}{l}
$\overline{\text { Algorithm } 6 \text { Non-linear version of Algorithm } 3}$ \\
\hline For $n>r$ :
\end{tabular}

1. Interface update: $\boldsymbol{\phi}_{h}^{n-1}=\boldsymbol{I}_{\Sigma}+\boldsymbol{d}_{h}^{n-1}, \Sigma_{h}^{n}=\phi_{h}^{n-1}(\Sigma), \Omega_{h}^{n}=\Omega \backslash\left(\Sigma_{h}^{n} \cup \widetilde{\Sigma}_{h}^{n}\right)$.

2. Find $\left(\boldsymbol{u}_{h}^{n}, p_{h}^{n}, \dot{\boldsymbol{d}}_{h}^{n-\frac{1}{2}}\right) \in \boldsymbol{V}_{h} \times Q_{h} \times \boldsymbol{W}_{h}$ such that

$$
\begin{aligned}
& \rho^{\mathrm{f}}\left(\partial_{\tau} \boldsymbol{u}_{h}^{n}, \boldsymbol{v}_{h}\right)_{\Omega}+a_{h}^{\mathrm{f}, n}\left(\boldsymbol{u}_{h}^{n} ;\left(\boldsymbol{u}_{h}^{n}, p_{h}^{n}\right),\left(\boldsymbol{v}_{h}, q_{h}\right)\right)+\frac{\rho^{\mathrm{s}} \epsilon}{\tau}\left(\dot{\boldsymbol{d}}_{h}^{n-\frac{1}{2}}, \boldsymbol{w}_{h}\right)_{\Sigma} \\
& -\sum_{i=1}^{2}\left(\boldsymbol{\sigma}\left(\boldsymbol{u}_{i, h}^{n}, p_{i, h}^{n}\right) \boldsymbol{n}_{i}, \boldsymbol{v}_{i, h}-\boldsymbol{w}_{h}\right)_{\Sigma_{h}^{n}}-\sum_{i=1}^{2}\left(\boldsymbol{u}_{i, h}^{n}-\dot{\boldsymbol{d}}_{h}^{n-\frac{1}{2}}, \boldsymbol{\sigma}\left(\boldsymbol{v}_{i, h},-q_{i, h}\right) \boldsymbol{n}_{i}\right)_{\Sigma_{h}^{n}} \\
& \left.+\sum_{i=1}^{2} \frac{\gamma \mu}{h}\left(\boldsymbol{u}_{i, h}^{n}-\dot{\boldsymbol{d}}_{h}^{n-\frac{1}{2}}, \boldsymbol{v}_{i, h}-\boldsymbol{w}_{h}\right)_{\Sigma_{h}^{n}}-\left(\llbracket \boldsymbol{\sigma}\left(\boldsymbol{u}_{h}^{n}, p_{h}^{n}\right)\right\} \boldsymbol{n}, \llbracket \boldsymbol{v}_{h} \rrbracket\right)_{\widetilde{\Sigma}_{h}^{n}} \\
& -\left(\left\{\boldsymbol{\sigma}\left(\boldsymbol{v}_{h},-q_{h}\right)\right\} \boldsymbol{n}, \llbracket \boldsymbol{u}_{h}^{n} \rrbracket\right)_{\widetilde{\Sigma}_{h}^{n}}+\frac{\gamma \mu}{h}\left(\llbracket \boldsymbol{u}_{h}^{n} \rrbracket, \llbracket \boldsymbol{v}_{h} \rrbracket\right)_{\widetilde{\Sigma}_{h}^{n}}=\frac{\rho^{\mathrm{s} \epsilon}}{\tau}\left(\dot{\boldsymbol{d}}_{h}^{n-1}+\tau \partial_{\tau} \dot{\boldsymbol{d}}_{h}^{n, \star}, \boldsymbol{w}_{h}\right)_{\Sigma} \\
& +\left(\llbracket \boldsymbol{\sigma}\left(\boldsymbol{u}_{h}^{n, \star}, p_{h}^{n, \star}\right) \boldsymbol{n} \rrbracket, \boldsymbol{w}_{h}\right)_{\Sigma_{h}^{n}}-\frac{2 \gamma \mu}{h}\left(\left\{\boldsymbol{u}_{h}^{n, \star}\right\}-\dot{\boldsymbol{d}}_{h}^{n-\frac{1}{2}, \star}, \boldsymbol{w}_{h}\right)_{\Sigma_{h}^{n}}
\end{aligned}
$$

for all $\left(\boldsymbol{v}_{h}, q_{h}, \boldsymbol{w}_{h}\right) \in \boldsymbol{V}_{h}^{n} \times Q_{h}^{n} \times \boldsymbol{W}_{h}$.

3. Find $\left(\dot{\boldsymbol{d}}_{h}^{n}, \boldsymbol{d}_{h}^{n}\right) \in \boldsymbol{W}_{h} \times \boldsymbol{W}_{h}$ with $\dot{\boldsymbol{d}}_{h}^{n}=\partial_{\tau} \boldsymbol{d}_{h}^{n}$ and such that

$$
\begin{aligned}
\rho^{\mathrm{s}} \epsilon\left(\partial_{\tau} \dot{\boldsymbol{d}}_{h}^{n}, \boldsymbol{w}_{h}\right)_{\Sigma}+a^{\mathrm{S}}\left(\boldsymbol{d}_{h}^{n}, \boldsymbol{w}_{h}\right)=-\left(\llbracket \boldsymbol{\sigma}\left(\boldsymbol{u}_{h}^{n}, p_{h}^{n}\right) \boldsymbol{n} \rrbracket, \boldsymbol{w}_{h}\right)_{\Sigma_{h}^{n}} & \\
& +\frac{2 \gamma \mu}{h}\left(\left\{\boldsymbol{u}_{h}^{n}\right\}-\dot{\boldsymbol{d}}_{h}^{n-\frac{1}{2}}, \boldsymbol{w}_{h}\right)_{\Sigma_{h}^{n}}
\end{aligned}
$$

for all $\boldsymbol{w}_{h} \in \boldsymbol{W}_{h}$.

The stability results of Theorems 2.2 2.4 remain valid for Algorithm 4 Algorithm 5 with $K=0$ and $r=1$, and Algorithm 6, respectively. The proofs follow by combining the identity 35. with the result of Lemma 2.1 and the arguments used in the proofs of the static case (Section 2.2. This shows that the explicit treatment of the interface location in the fluid, (34), does not compromise the energy stability of the methods. Similar conclusions are known for semi-implicit time discretizations of the immersed boundary method (see, e.g., [56, 8]).

It should be noted, however, that the semi-implicit schemes reported in Algorithm 6 have a reduced computational complexity with respect to alternative semi-implicit schemes used in the immersed boundary method (see, e.g., [56, 8]), which treat the fluid-solid kinematic-dynamic coupling $[30]_{1,3}$ in a fully implicit fashion (strong coupling), as Algorithm 4

\subsubsection{Integrals with discontinuous functions at different time levels}

At each time level, the first step of Algorithms 4 involves the computation of the new interface intersections and the new sub-divisions of the cut-elements. In other words, we perform the intersection algorithm of Section 2.1.4 at each time-step. Some of the fluid integrals in Algorithms 46 . however, involve fluid discrete functions associated with different time levels, namely, 
the bulk term related to the time-stepping scheme in the fluid,

$$
\frac{\rho^{\mathrm{f}}}{\tau}\left(\boldsymbol{u}_{h}^{n-1}, \boldsymbol{v}_{h}\right)_{\Omega},
$$

and the interface terms

$$
\left(\boldsymbol{\sigma}\left(\boldsymbol{u}_{i, h}^{n-1}, p_{i, h}^{n-1}\right) \boldsymbol{n}_{i}, \boldsymbol{v}_{i, h}\right)_{\Sigma_{h}^{n}}, \quad \frac{\gamma_{0} h}{\gamma \mu}\left(p_{i, h}^{n-1}, q_{i, h}\right)_{\Sigma_{h}^{n}},
$$

associated with the fluid-solid splitting of Algorithm 5. A practical difficulty arises whenever

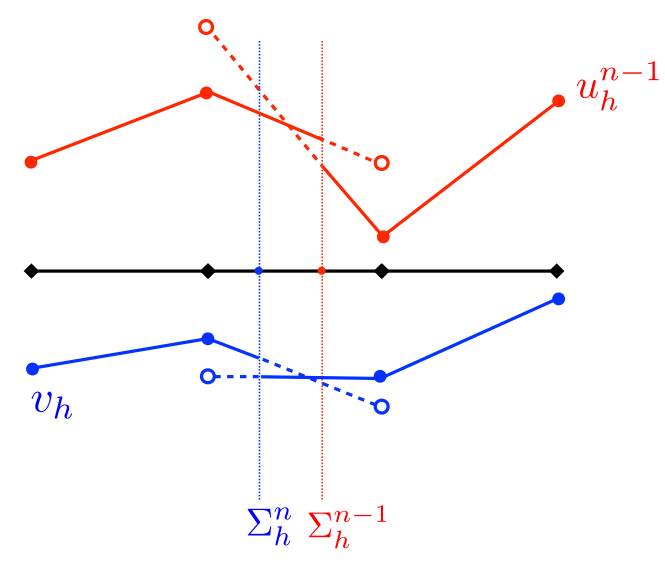

(a)

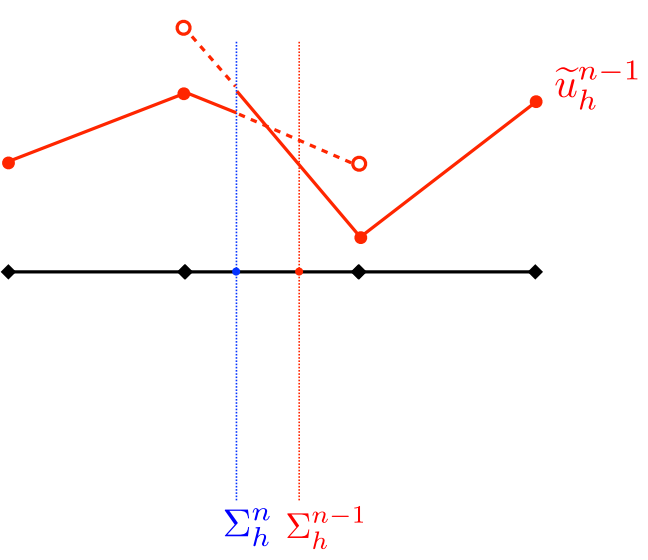

(b)

Figure 8: he previous solution $u_{h}^{n-1} \in V_{h}^{n-1}$ and the test function $v_{h} \in V_{h}^{n}$ are discontinuous at different locations, $\Sigma_{h}^{n-1}$ and $\Sigma_{h}^{n}$ respectively, within the same element. The dotted lines represent the value of the functions in the fictitious zone of the overlapping region and the circles the ghost values (a). Approximation of the function $u_{h}^{n-1}$ with the discontinuity at $\Sigma_{h}^{n}$ (b).

$\left(\boldsymbol{u}_{h}^{n-1}, p_{h}^{n-1}\right) \in \boldsymbol{V}_{h}^{n-1} \times Q_{h}^{n-1}$ and $\left(\boldsymbol{v}_{h}, q_{h}\right) \in \boldsymbol{V}_{h}^{n} \times Q_{h}^{n}$ are discontinuous at different locations within the same element. This issue is illustrated in 1D in Figure 8(a), which shows that the evaluation of the bulk terms is much more involved than in the static framework of Section 2 , since the interface locations at $t_{n-1}$ and $t_{n}$ have to be considered in the evaluation of the intersections and sub-divisions of the same element. Instead, we propose to approximate the quantities defined at $t_{n-1}$ by shifting the discontinuity to the location of the interface at $t_{n}$ (see Figure $8(\mathrm{~b})$ ). Basically, this amounts to evaluate part of the functions defined at $t_{n-1}$ in the fictitious zone of the overlapping region. In this sense, it can be viewed as a class of ghost fluid method (see, e.g., [29]). A similar approach is discussed in [35] (therein called Alternative 2) in the framework of time-stepping schemes for XFEM methods with dynamic interfaces.

\section{$4 \quad$ Numerical examples}

The purpose of this section is to illustrate, via a series of $2 \mathrm{D}$ numerical examples, the stability and accuracy of the methods introduced in Sections 2.2 and 3.2 . We consider a simple string model for the solid, so that in (2) and $(30)$ we have

$$
\boldsymbol{d}=\eta \boldsymbol{n}, \quad \boldsymbol{L} \boldsymbol{d}=\left(-\lambda_{1} \partial_{s}^{2} \eta+\lambda_{0} \eta\right) \boldsymbol{n},
$$

where $\eta: \Sigma \times \mathbb{R}^{+} \rightarrow \mathbb{R}$ denotes the normal displacement and $\lambda_{1}, \lambda_{0}>0$ are given coefficients. 


\subsection{Static interface}

We first consider the case of the linear model problem of Section 2 (static interface). The next two paragraphs illustrate numerically the accuracy properties of Algorithms 13 in two different configurations. We retrieve, in particular, the convergence rates reported in Section 2.2 Along this section, the user-defined parameters of Algorithms 13 are fixed to $\gamma_{\mathrm{p}}=10^{-3}, \gamma_{\mathrm{g}}=1$, $\gamma=10^{3}$ and (in Algorithm 2) $\gamma_{0}=1$.

\subsubsection{Idealized closed valve}

The purpose of this first example is to mimic the behavior of a closed valve under a given pressure drop. We consider a rectangular fluid domain with the thin-walled solid immersed along its middle cross section (see Figure 9). We take $\Omega=(0,4) \times(0,1)$ and $\Sigma=\{2\} \times(0,1)$ in (1)-2). All the units are given in the CGS system. The fluid physical parameters are $\rho^{\mathrm{f}}=1$ and $\mu=0.035$. For the solid we have $\rho^{\mathrm{s}}=1.1, \epsilon=0.1, \lambda_{1}=E \epsilon /(2(1+\nu))$ and $\lambda_{0}=0$, with Young's modulus $E=0.75 \cdot 10^{6}$ and Poisson's ratio $\nu=0.5$. The external boundary conditions for the fluid problem are shown in Figure 9. A steady pressure drop of magnitude $P_{0}=20000$ is enforced between the fluid inlet and outlet boundaries. The structure is fixed on its extremities.

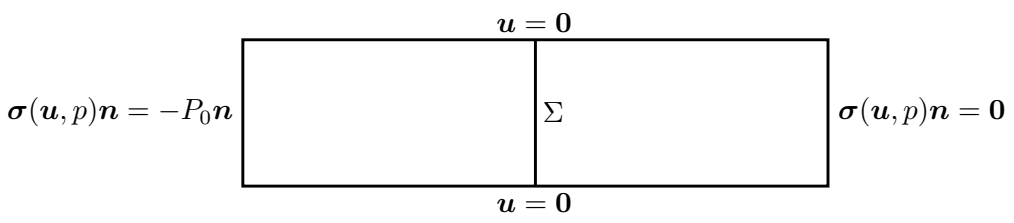

Figure 9: Geometric configuration and external boundary conditions.

After a brief transition phase, the system reaches a steady state with a pressure jump across the interface. For this simple configuration, the exact solution can be computed analytically. The fluid and solid velocities vanish and the pressure is a piecewise constant function taking the value $P_{0}$ on the left side and zero on the right one. Hence, the solid equation reduces to the single $1 \mathrm{D}$ boundary value problem

$$
-\lambda_{1} \partial_{y}^{2} \eta=\llbracket p \rrbracket=P_{0} \quad \text { in } \quad(0,1), \quad \eta(0)=\eta(1)=0,
$$

whose solution is given by

$$
\eta(y)=\frac{P_{0}}{2 \lambda_{1}} y(1-y)
$$

This analytic solution is used to evaluate the spatial accuracy of the methods.

Because of the time independent nature of the problem, we limit the discussion to the implicit scheme given by Algorithm[1. We consider four pairs of unfitted fluid-solid meshes with increasing degree of refinement, namely,

$$
h \in\left\{0.1 / 2^{i}\right\}_{i=0}^{3} .
$$

For illustration purposes, we have displayed in Figure 10 the steady state displacement provided by Algorithm 1 for $h=0.1$, together with the analytical solution 40 and the approximation obtained with an implicit fitted method. Note that both the fitted and the unfitted approximations perfectly match the analytical solution. The corresponding pressure fields are reported in Figure 11. The unfitted method is able to perfectly capture the pressure drop without spurious oscillations. The overlap of the approximation at the interfacial zone is also clearly visible. 


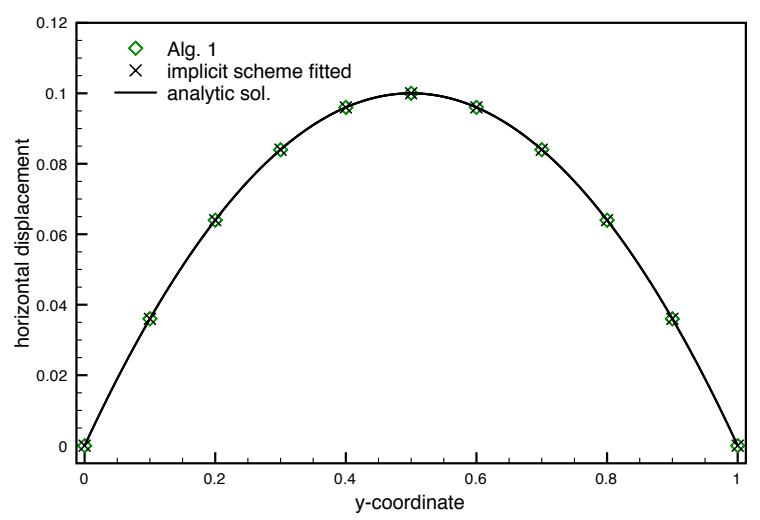

Figure 10: Steady state displacement.

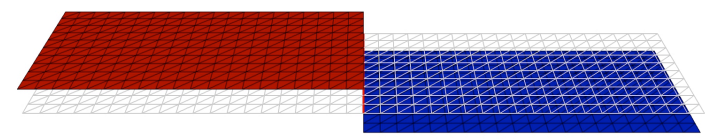

(a) Fitted method

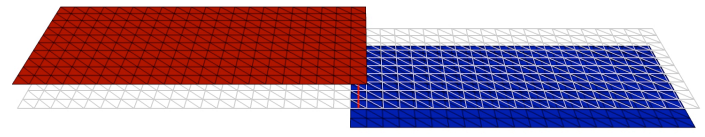

(b) Algorithm 1

Figure 11: Steady state pressure approximations.

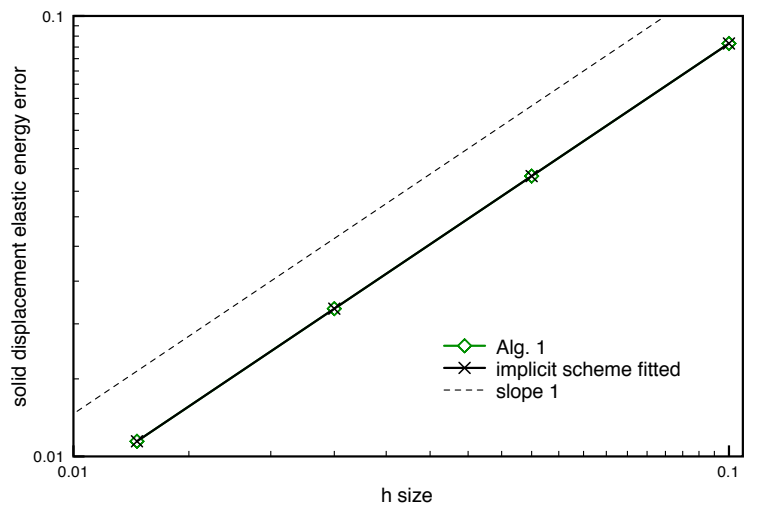

Figure 12: Convergence history in space.

Finally, Figure 12 presents the convergence history of the displacement approximations in the energy norm, for the fitted and the unfitted methods. As expected, we retrieve the optimal first-order convergence rate predicted by Theorem 2.2 for Algorithm 1.

\subsubsection{Pressure-waves}

This example is a variant of the well-known fluid-structure benchmark describing the propagation of a pressure-wave within an elastic tube. The fluid domain is given by the rectangle $\Omega=$ $(0,6) \times(0,0.8)$ and the interface by the segment $\Sigma=(0,6) \times\{0.54\}$ (see Figure 13). As in the previous example, all the units are given in the CGS system. The fluid physical parameters 
are $\rho^{\mathrm{f}}=1.0, \mu=0.035$. For the solid we have $\rho^{\mathrm{s}}=1.1, \epsilon=0.1, \lambda_{1}=E \epsilon /(2(1+\nu))$ and $\lambda_{0}=E \epsilon /\left(0.25\left(1-\nu^{2}\right)\right)$, with Young's modulus $E=0.75 \cdot 10^{6}$ and Poisson's ratio $\nu=0.5$. The boundary conditions for the fluid are reported in Figure 13 . On the inlet boundary $\{0\} \times[0,0.54]$ we impose a sinusoidal pressure $p_{\text {in }}(t)$, of maximal amplitude $2 \cdot 10^{4}$, during $5 \cdot 10^{-3}$ seconds (half a period). The solid is clamped on its extremities.

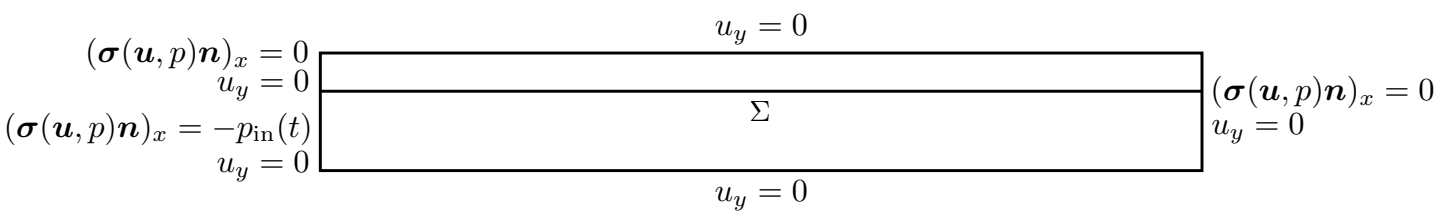

Figure 13: Geometric configuration and external boundary conditions.

Figure 14 shows some snapshots of the elevated pressure field at three different time instants obtained with an implicit fitted method and Algorithm 1. The zooms included in Figures 14(a) and 14(b) illustrate the fitted and unfitted nature of the meshes. Algorithm 1 gives practically the same accuracy as the fitted method, predicting the propagation of a pressure jump along the tube.
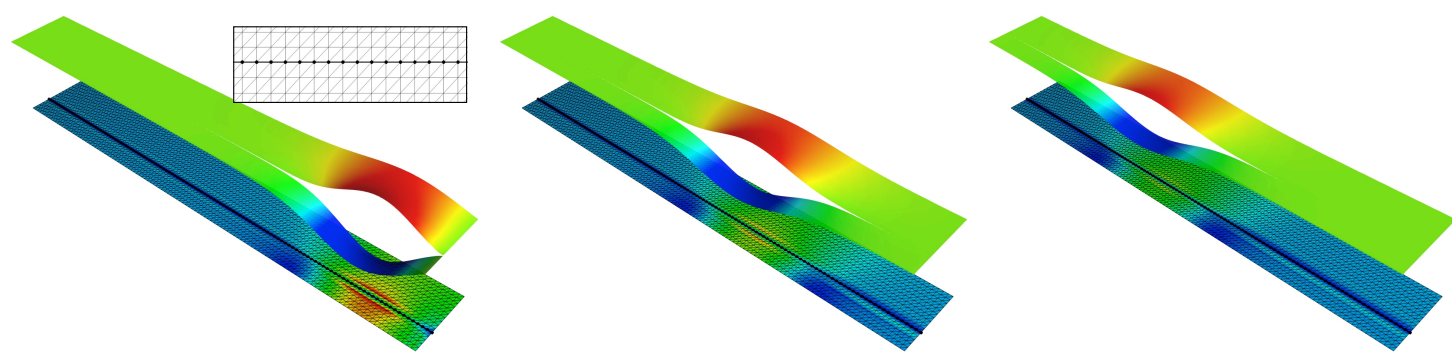

(a) Fitted method
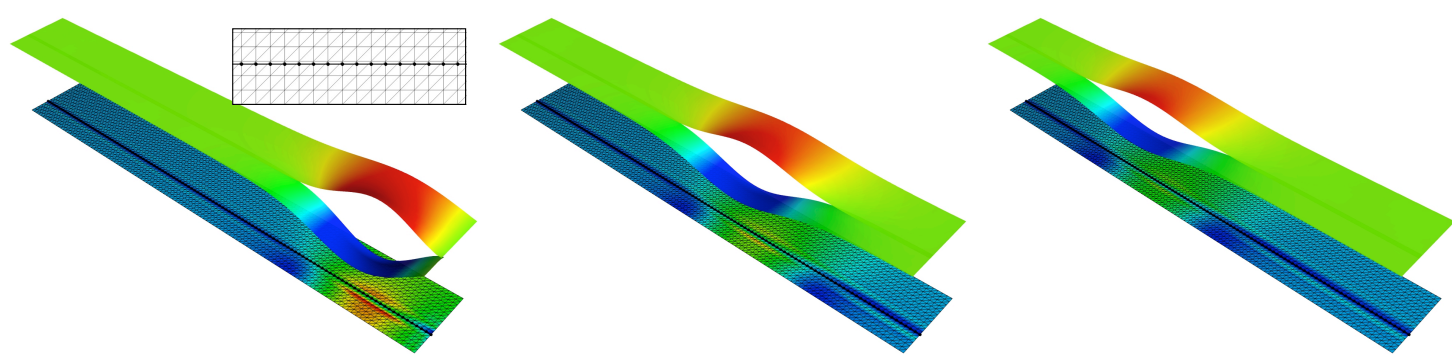

(b) Algorithm 1
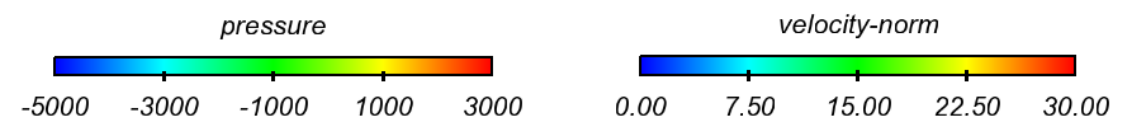

Figure 14: Snapshots of the fluid velocity magnitude and elevated pressure at time $t=$ $0.005,0.01,0.015$ (from left to right) obtained with $h=0.05$ and $\tau=10^{-4}$.

In order to provide numerical evidence on the convergence rates of Algorithms 1 3 , we have 


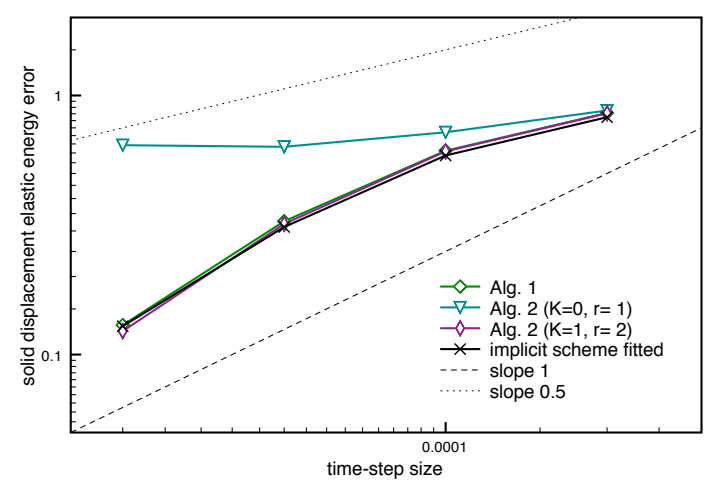

(a) Algorithms 1 and 2

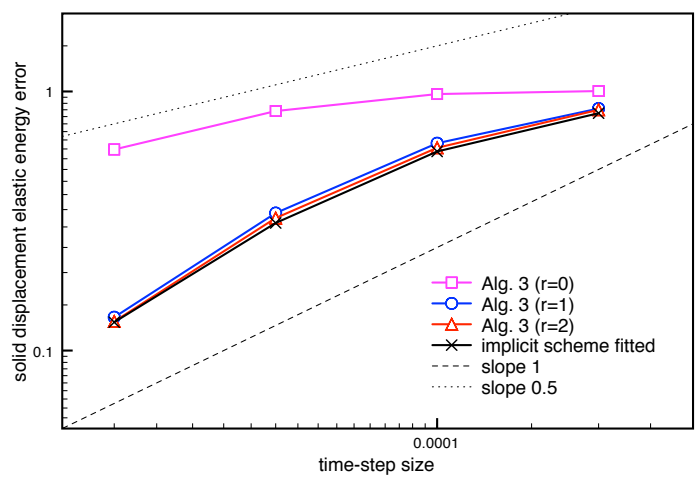

(b) Algorithm 3

Figure 15: Time-convergence history of the displacement at $t=0.015$ obtained with $h=\mathcal{O}(\tau)$.

uniformly refined both in time and in space according to the parameters

$$
(\tau, h) \in\left\{\left(2 \cdot 10^{-4} / 2^{i}, 0.1 / 2^{i}\right)\right\}_{i=0}^{3} .
$$

Note that $\tau=\mathcal{O}(h)$. A reference solution has been generated with the fitted implicit method using $\tau=6.25 \cdot 10^{-6}$ and $h=3.125 \cdot 10^{-3}$ (i.e., $i=5$ in (41)). Figure 15 reports the convergence histories of the displacement error in the elastic energy norm at $t=0.015$ obtained with Algorithms 1 3 . For comparison purposes, the convergence history of the fitted implicit approximations is also displayed. The corresponding displacements, for each level of space-time refinement, are reported in Figures 16 and 17.

Figure 15(a) retrieves the first-order optimal convergence rate predicted by Theorem 2.2 for Algorithm 11. This convergent behavior is also clearly visible in Figure 16, which points out the good agreement with the approximations provided by the implicit fitted method. As regards Algorithm 2 Figure 15(a) indicates that the variant without extrapolation $(K=0, r=1)$ fails to converge under $\tau=\mathcal{O}(h)$. On the contrary, stable and first-order accurate approximations are obtained with a second-order prediction and one correction iteration $(K=1, r=2)$. This behavior is also clearly visible in Figure 16, hence confirming the theoretical discussion on the accuracy of the methods of Section 2.2 .2 .

Finally, we comment on the results obtained with Algorithm 3 Figure 15(b) shows convergence for the three variants. Note, however, that sub-optimal accuracy is obtained with the variant without extrapolation $(r=0)$. This behavior is also striking in Figure 17, which points out the extremely poor accuracy of the displacement approximations for all the space-time refinement levels. On the contrary, the variants with $r=1$ and $r=2$ retrieve the optimal firstorder accuracy of the fitted implicit scheme, hence confirming the convergence rates reported in Theorem 2.4 .

\subsection{Dynamic interface}

In this section we provide numerical evidence on the stability and the accuracy properties of Algorithms 46 in different examples. For comparison purposes, we consider as reference solution an implicit fitted-ALE approximation of problem (33), where the discrete ALE mapping is built from a simple harmonic lifting of the solid displacement (no advanced mesh update strategy is hence used). Along this section, the user-defined parameters in Algorithms 46 are always 


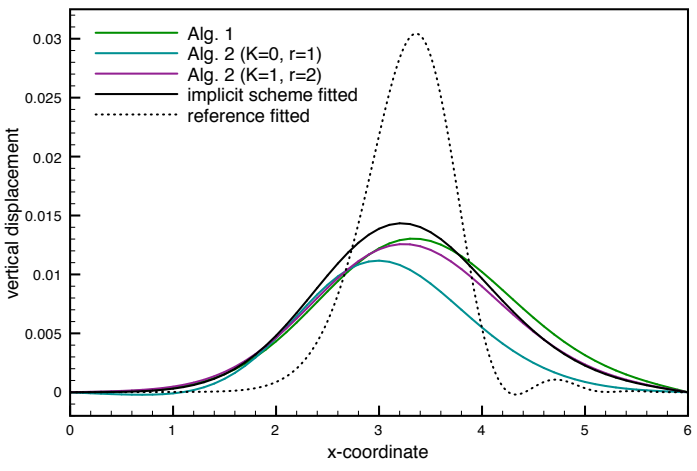

(a) $i=0$.

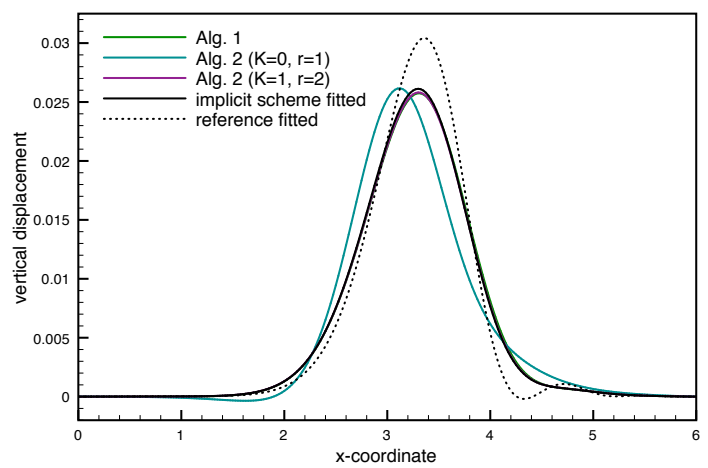

(c) $i=2$.

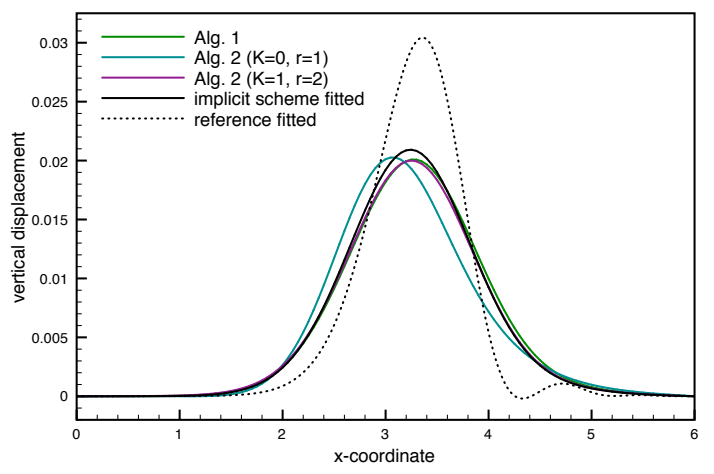

(b) $i=1$.

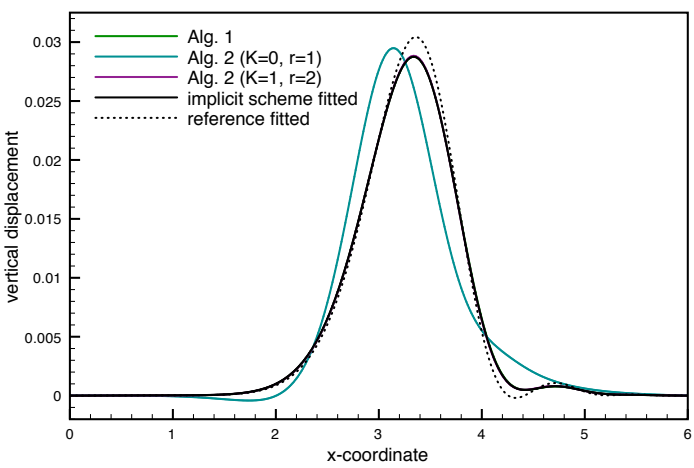

(d) $i=3$.

Figure 16: Algorithms 1 and 2. Comparison of the solid displacements at $t=0.015$ for different levels of $(\tau, h)$-refinement 41$)$.

chosen to be $\gamma_{\mathrm{p}}=10^{-2}, \gamma_{\mathrm{v}, 1}=10^{-2}, \gamma_{\mathrm{v}, 2}=0, \gamma_{\mathrm{g}}=1, \gamma=10^{2}$ and (in Algorithm 5 ) $\gamma_{0}=1$. Furthermore, for simplicity, a standard semi-implicit treatment of the fluid convective term, $a_{h}^{\mathrm{f}, n}\left(\boldsymbol{u}_{h}^{n-1} ;\left(\boldsymbol{u}_{h}^{n}, p_{h}^{n}\right),\left(\boldsymbol{v}_{h}, q_{h}\right)\right)$, is adopted in Algorithms 46 .

\subsubsection{Idealized closed valve}

We consider the idealized closed valve test of Section 4.1.1 within the non-linear framework of Section 3. The geometrical configuration, physical parameters and boundary conditions are those of Section 4.1.1. A remarkable feature of this setting is that both the static and the dynamic interface cases share the same steady state displacement solution. Indeed, using the parameterization of the interface configuration $\Sigma(t)$, in terms of the steady state displacement, given by $\boldsymbol{r}(y)=(\eta(y), y), y \in[0,1]$, we have

$$
\begin{aligned}
-\int_{\Sigma(t)} & \llbracket \boldsymbol{\sigma}(\boldsymbol{u}, p) \boldsymbol{n} \rrbracket \cdot \boldsymbol{w} \circ \boldsymbol{\phi}_{t}^{-1}=\int_{0}^{1} P_{0} \boldsymbol{n}(\boldsymbol{r}(y)) \cdot \boldsymbol{w}\left(\phi_{t}^{-1}(\boldsymbol{r}(y))\right)\left|\boldsymbol{r}^{\prime}(y)\right| \mathrm{d} y \\
& =\int_{0}^{1} P_{0}\left|\boldsymbol{r}^{\prime}(y)\right|^{-1}\left(1,-\eta^{\prime}(y)\right) \cdot(w(y), 0)\left|\boldsymbol{r}^{\prime}(y)\right| \mathrm{d} y=\int_{0}^{1} P_{0} w(y) \mathrm{d} y
\end{aligned}
$$




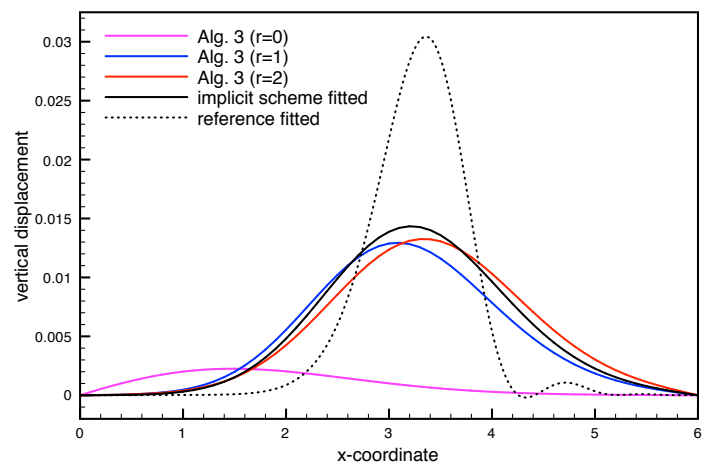

(a) $i=0$.

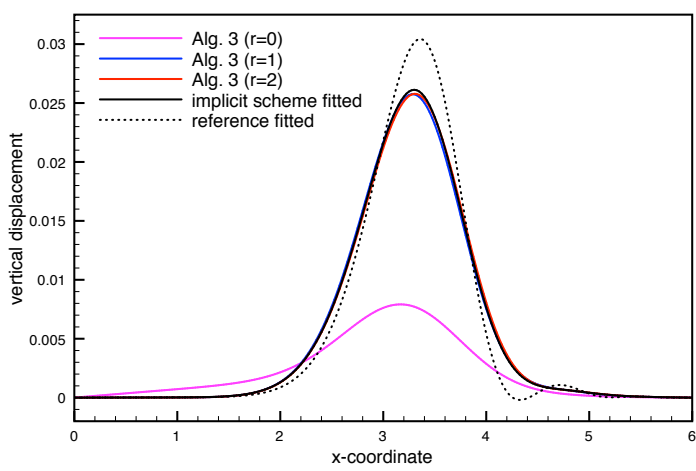

(c) $i=2$.

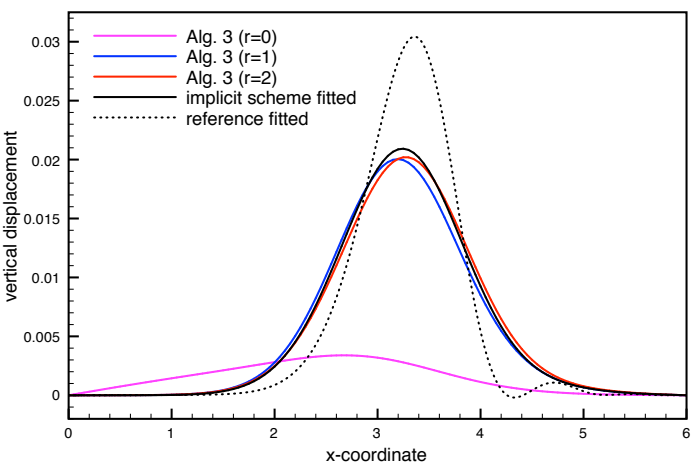

(b) $i=1$.

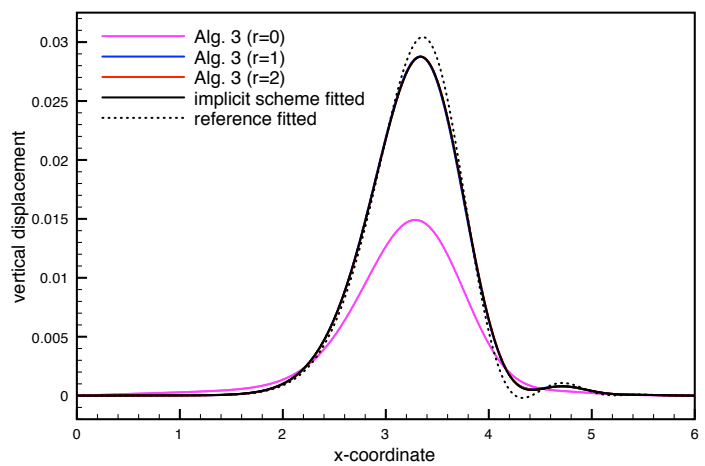

(d) $i=3$.

Figure 17: Algorithm 3 Comparison of the solid displacements at $t=0.015$ for different levels of $(\tau, h)$-refinement 41$)$.

for all $\boldsymbol{w} \in \boldsymbol{W}$. We hence recover (39), as the strong form of (30), and the analytical expression for the displacement given by 40 .

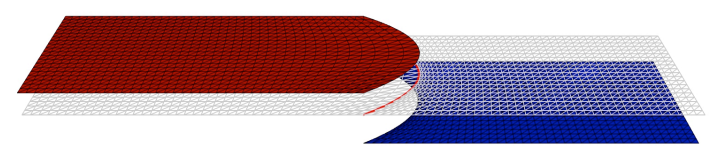

(a) Fitted method

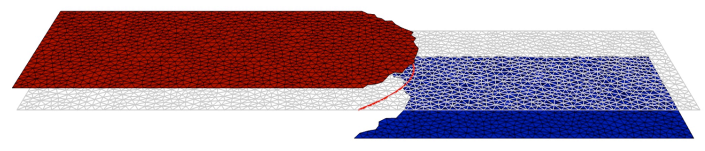

(b) Algorithm 4

Figure 18: Steady state pressure approximation and deformed solid configuration.

Due to the stationary nature of the problem, we limit the discussion to the implicit scheme given by Algorithm 4, and compare its accuracy with the implicit ALE-fitted method. Prototypical approximations obtained with both approaches are displayed in Figure 18 . The mesh size for the fitted (structured) and the unfitted (unstructured) meshes is approximately $h \approx 0.05$. Note the distortion of the fluid mesh in Figure 18(a), prescribed by the ALE fitted approach, in order to fit the solid mesh. In Figure 18(b), on the contrary, the structure mesh moves independently of the fluid background mesh. This is more visible in Figure 19, which presents the 
mesh intersection and the cut-elements subdivisions resulting from the algorithm described in Section 2.1.4.

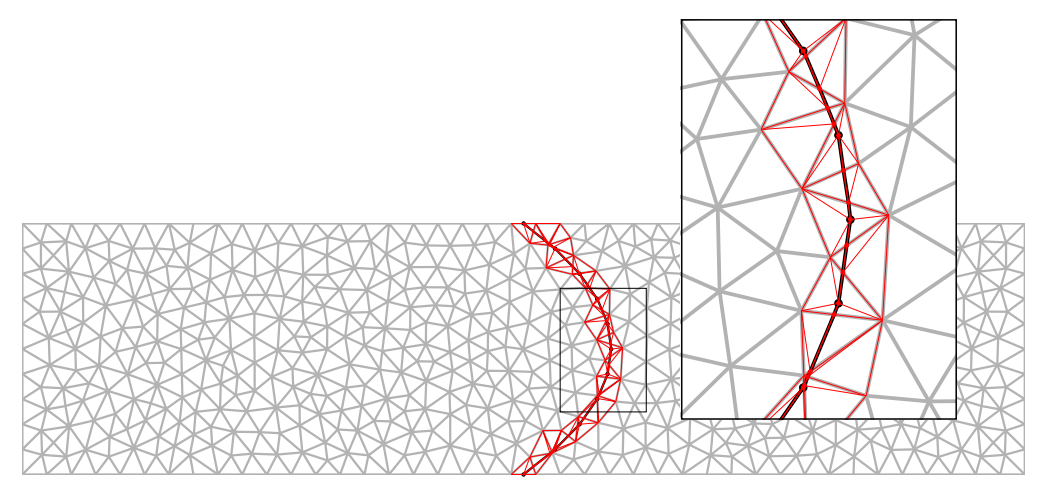

Figure 19: Mesh intersection and the cut-elements subdivisions.

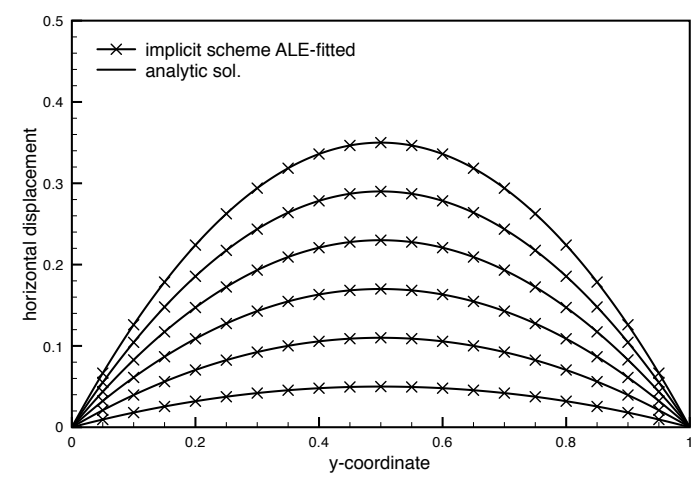

(a) Fitted method

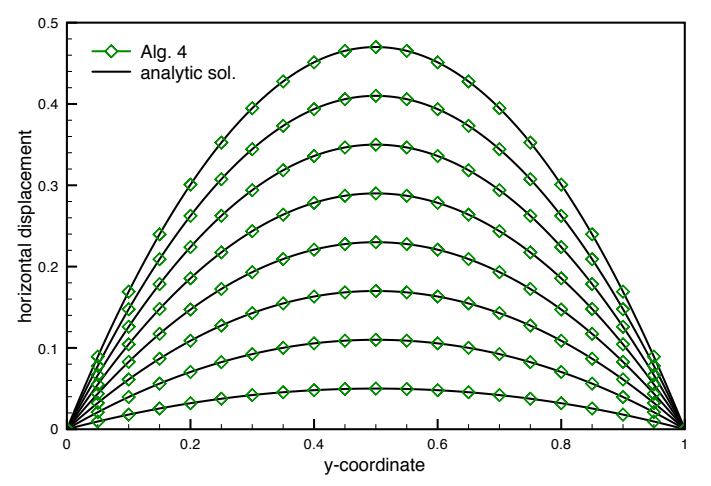

(b) Algorithm 4

Figure 20: Steady state displacements for increasing inlet pressures.

In order to show the capabilities of the proposed unfitted methods to handle situations with large interface displacements, we consider a series of increasing inlet pressures $P_{0} \in\left\{10^{4}+12 \cdot 10^{3}\right.$. $i\}_{i=0}^{7}$. In Figure 20, we have reported the displacement approximations obtained with the fitted and the unfitted methods. The analytical solutions given by (40) are also displayed. We observe that both approaches are in excellent agreement with the analytical solution until $i=5$. From this point on, the considered ALE mesh update fails. On the contrary, the unfitted approach is able to continue for $i \geq 6$ while keeping a perfect match with the analytic solution.

\subsubsection{Double cavity}

We consider a variant of the classical lid-driven cavity problem in which a second cavity is attached below the lower flexible wall. The fluid domain is given by the rectangle $\Omega=(-0.5,0.5) \times$ $(-0.5,1.5)$ and the interface by the segment $\Sigma=(-0.5,0.5) \times\{0.5\}$ (see Figure 21). All units are given in the SI system.

The fluid physical parameters are given by $\rho^{\mathrm{f}}=1.0$ and $\mu=0.2$. For the solid we have $\rho^{\mathrm{s}}=250, \epsilon=0.01, \lambda_{1}=E \epsilon /(2(1+\nu))$ and $\lambda_{0}=0$, with $\nu=0$ and we consider three different 


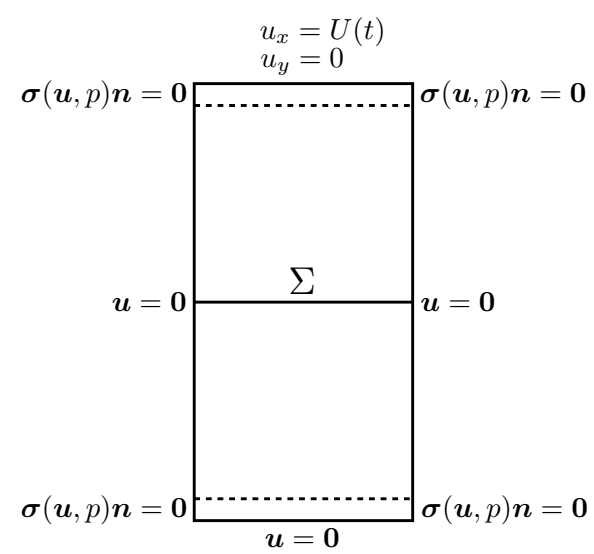

Figure 21: Geometric configuration and external boundary conditions.
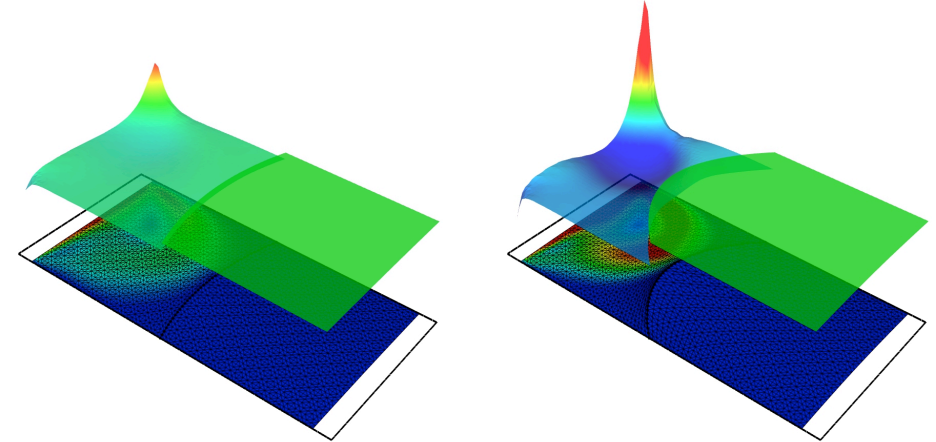

(a) ALE-fitted method
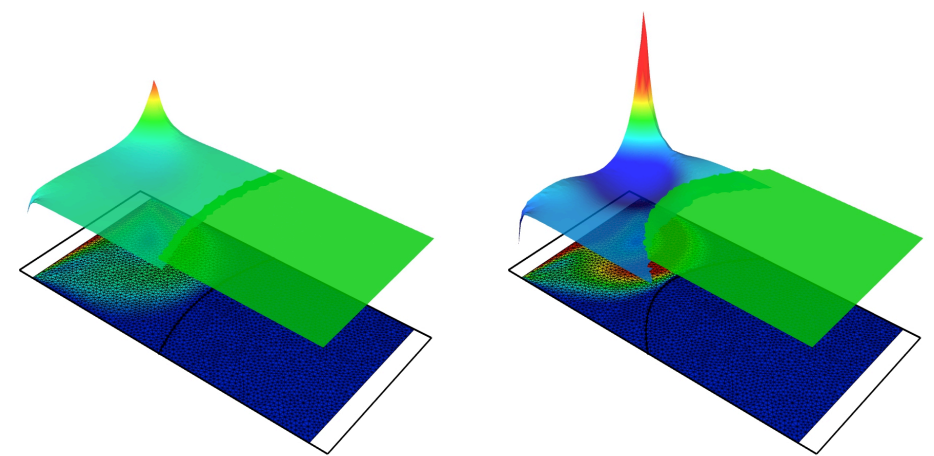

(b) Algorithm 4
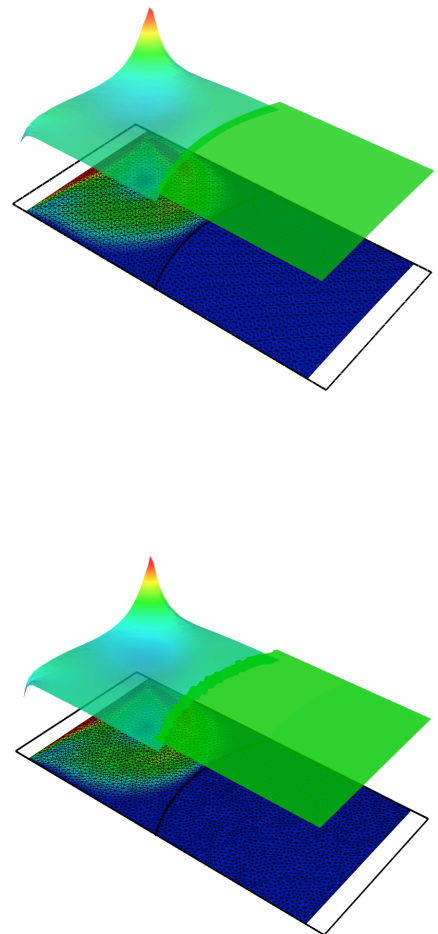
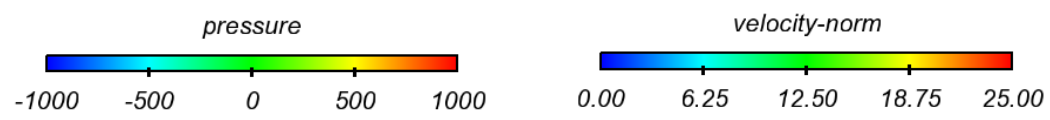

Figure 22: Snapshots of the fluid velocity magnitude and elevated pressure at time $t=6.25,7.5$ and 8.75 (from left to right) obtained with $E=62500, \tau=5 \cdot 10^{-3}$ and $h \approx 0.025$. 


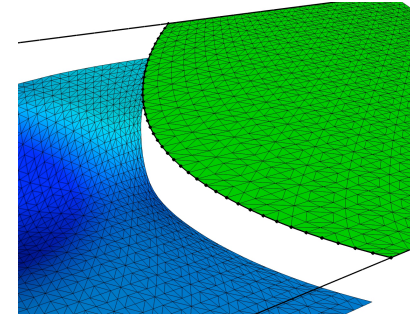

(a) Pressure

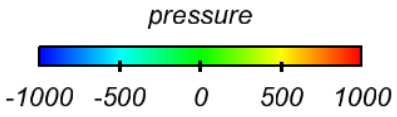

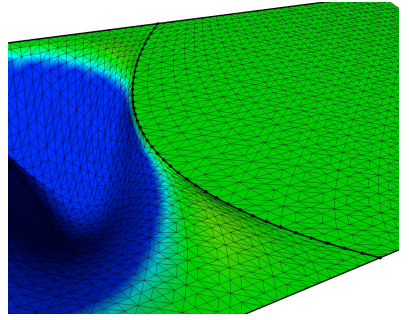

(b) Horizontal velocity horizontal velocity

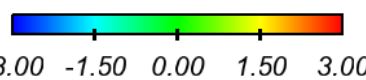

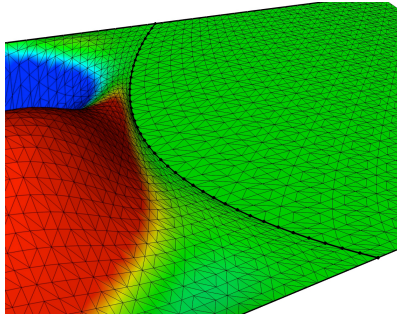

(c) Vertical velocity vertical velocity

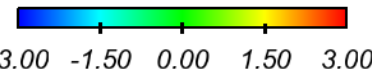

Figure 23: ALE-fitted method. Strong (pressure) and weak (velocity) discontinuities of the approximated solution at time $t=7.5$ with $E=62500, \tau=5 \cdot 10^{-3}$ and $h \approx 0.025$.

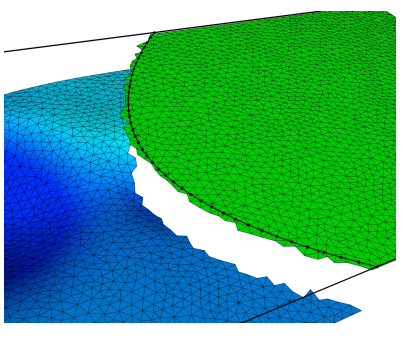

(a) Pressure

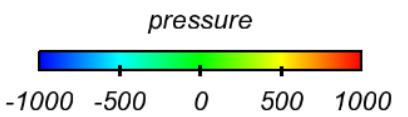

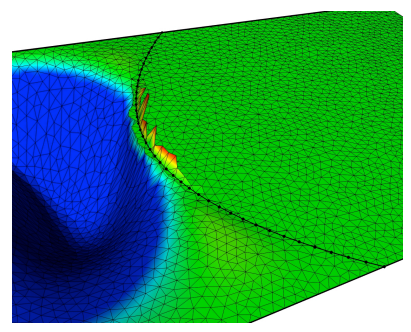

(b) Horizontal velocity

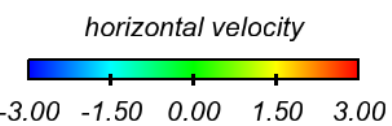

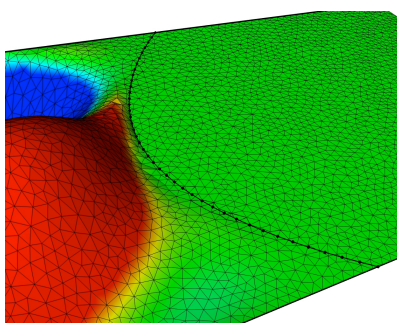

(c) Vertical velocity

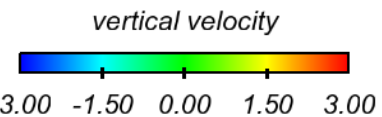

Figure 24: Algorithm 4. Strong (pressure) and weak (velocity) discontinuities of the approximated solution at time $t=7.5$ with $E=62500, \tau=5 \cdot 10^{-3}$ and $h \approx 0.025$.

values for the Young modulus, $E=\left\{125000 / 2^{i}\right\}_{i=0}^{2}$. The fluid external boundary conditions are given in Figure 21 with $U(t) \stackrel{\text { def }}{=} 100[1-\cos (0.4 \pi t)]$. The solid is clamped on its extremities.

Figure 22 presents some snapshots of the fluid velocity magnitude and of the elevated fluid pressure obtained with the ALE-fitted method and Algorithm 4 for a value of the Young modulus, $E=62500$, which gives relatively large interface displacements. The time-step length is $\tau=0.005$ and the mesh step is approximately $h \approx 0.025$ in both simulations. The good agreement between the fitted and unfitted approaches is noticeable.

Figures 23 and 24 present a closer view of the discrete solutions at time $t=7.5$, showing the elevated pressure and velocity components. The comparison of these figures demonstrates the capability of the present unfitted approach to accurately capture the weak and strong discontinuities of the velocity and pressure fields across the interface. No spurious oscillations appear. Note that the unfitted approximation is two-valued in the set of elements intersected by the interface, only its value in the physical zone of the overlapping region must be compared with the results of Figures 23 .

In order to investigate the accuracy of the different coupling schemes, we have reported in Figures 25 and 26 the time history of the solid displacement at point $(-0.2,0.5)$ obtained with 


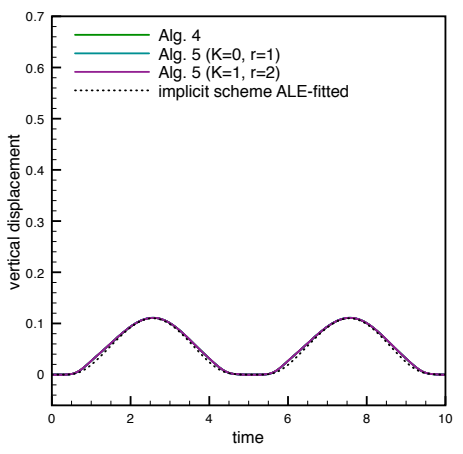

(a) $E=125000$

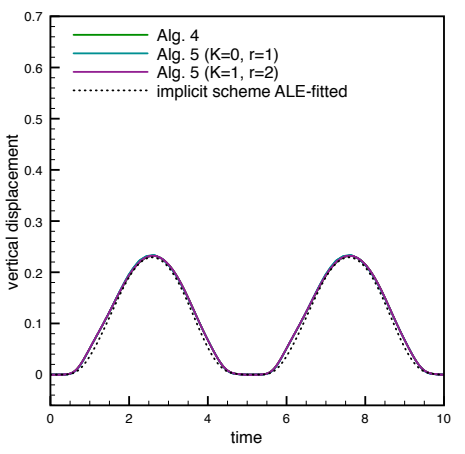

(b) $E=62500$

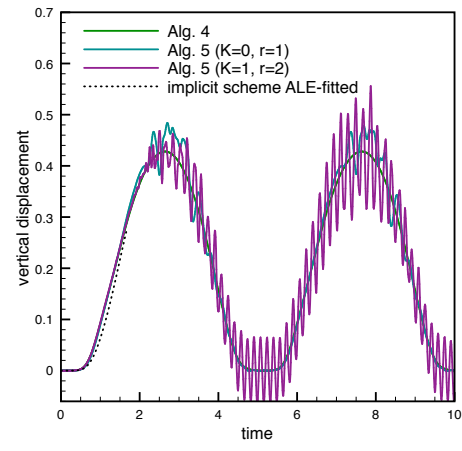

(c) $E=31250$

Figure 25: Time history of the solid displacement at point $(-0.2,0.5)$ obtained with Algorithms 4 and $5, \tau=5 \cdot 10^{-3}$.

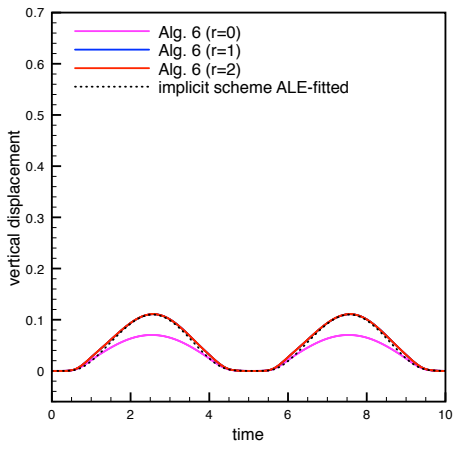

(a) $E=125000$

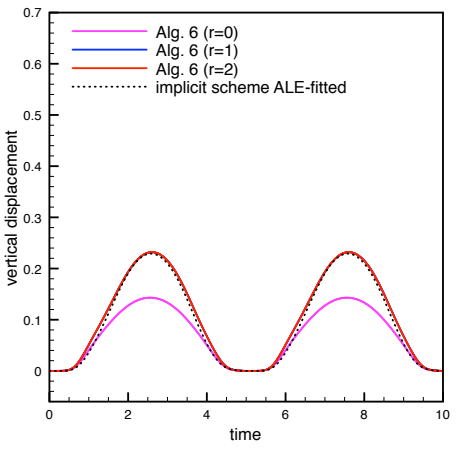

(b) $E=62500$

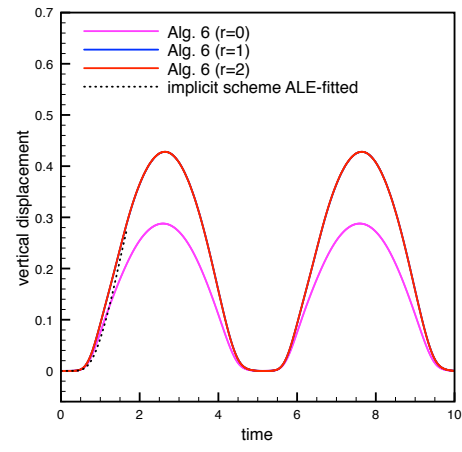

(c) $E=31250$

Figure 26: Time history of the solid displacement at point $(-0.2,0.5)$ obtained with Algorithm 6 . $\tau=5 \cdot 10^{-3}$.

Algorithms 45 and Algorithm6, respectively, for the three different values of the Young modulus. For comparison purposes, the corresponding results for the ALE-fitted method are also plotted.

Figure 25 shows that Algorithm 4 gives similar results as the ALE-fitted method in all the regimes. As expected, the higher the Young modulus, the lower the displacement amplitude. Note that for $E=31250$, the considered ALE-fitted method fails at a certain level of interface deformation (due to the breakdown of the mesh update technique), while Algorithm 4 does not show any lack of robustness.

The situation is more delicate for the explicit coupling schemes given by Algorithm 5. The two explicit variants do deliver stable approximations, but some spurious time oscillations appear in the case of the largest interface deflections. This issue is related to the non-uniformity in space of the splitting error discussed in Section 2.2.2. Indeed, as shown in Figure 27, reducing the timestep length $\tau$ (while keeping $h$ fixed) improves the quality of the approximations. The enhanced accuracy of the variant with second-order prediction and one correction iteration $(K=1, r=2)$ is also noticeable. Numerical evidence, not reported here, shows however that this variant may lack stability if $\gamma \tau / h$ is not sufficiently small. As an example, with $\gamma=1000$, as in Section 4.1 . stability requires more than one correction iteration $(K>1)$. 


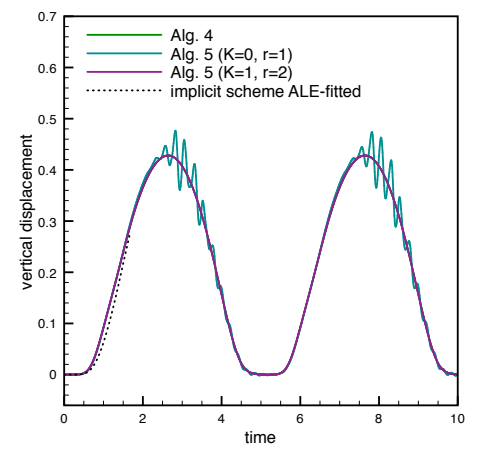

(a) $\tau=2.5 \cdot 10^{-3}$

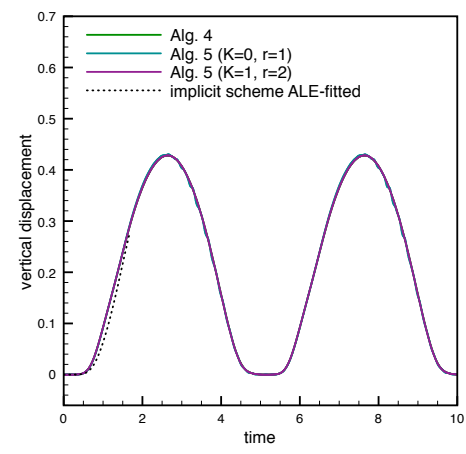

(b) $\tau=1.25 \cdot 10^{-3}$

Figure 27: Time history of the solid displacement at point $(-0.2,0.5)$ obtained with Algorithms 4 and $5, E=31250$.

The results reported in Figure 26 indicate that the semi-implicit schemes given by Algorithm6 with $r=1$ and $r=2$ do not suffer from the above issues. These variants deliver comparable accuracy to the implicit ALE-fitted method. On the contrary, the variant with $r=0$ provides poor approximations in all the regimes. We thus retrieve, also in the dynamic interface framework, the essential ingredients of the accuracy result given by Theorem 2.4

\subsubsection{Idealized open valve}

This example is intended to mimic the behavior of an open valve (without contact). The fluid domain corresponds to the rectangle $\Omega=(0,4) \times(0,1)$ and the solid domain is made of two segments $\Sigma=\{1.9\} \times(0,0.6) \cup\{2.1\} \times(0.4,1)$, as shown in Figure 28. All the units are given in the CGS system. The physical parameters for the fluid are $\rho^{\mathrm{f}}=1$ and $\mu=0.03$. For the

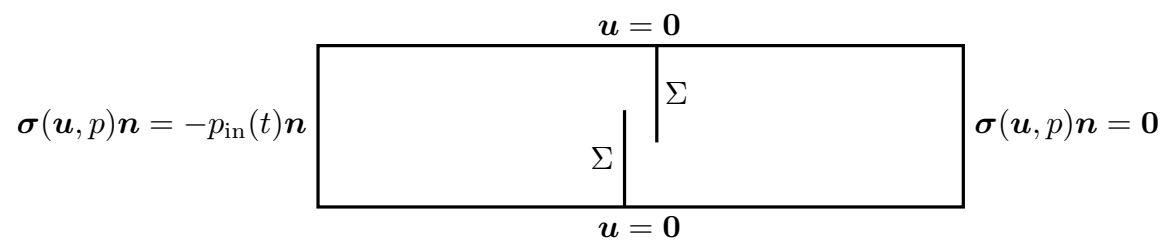

Figure 28: Geometric configuration and external boundary conditions.

structure we have $\rho^{\mathrm{s}}=1.2, \epsilon=0.065, \lambda_{1}=E \epsilon /(2(1+\nu))$ and $\lambda_{0}=0$, with Young's modulus $E=10000$ and Poisson's ratio $\nu=0.5$. The external boundary conditions for the fluid are detailed in Figure 28, with the inlet pressure given by $p_{\text {in }}(t) \stackrel{\text { def }}{=} P_{\max } \sin (2 \pi t)$ with $P_{\max }=150$. The solid is clamped at its bottom and top extremities, i.e., at $(1.9,0.6)$ and $(2.1,1)$.

Figure 29 presents some snapshots of the fluid velocity magnitude and of the elevated fluid pressure obtained with the ALE-fitted method and Algorithm 4. The time step is $\tau=0.0025$ and the step parameter of the fluid and solid meshes is approximately $h \approx 0.05$. The unfitted method is able to capture the dynamics of the pressure jump across the interface delivered by the fitted approximation without any spurious oscillation. This feature is even more striking in Figures 30 and 31. where we present a closer view of the elevated pressure and velocity 

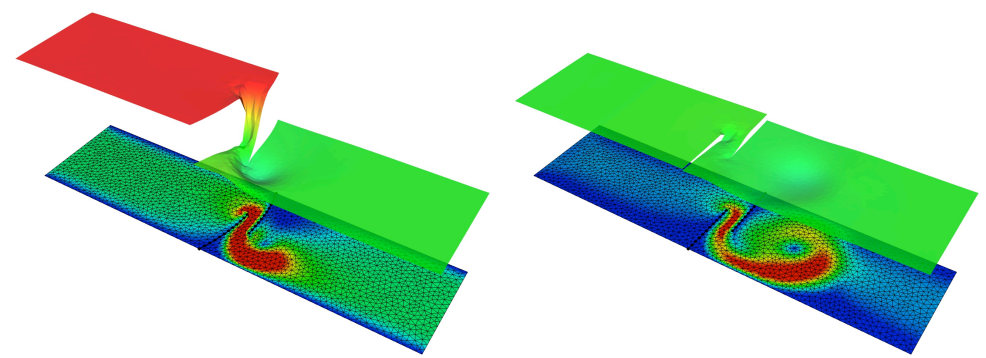

(a) ALE-fitted method
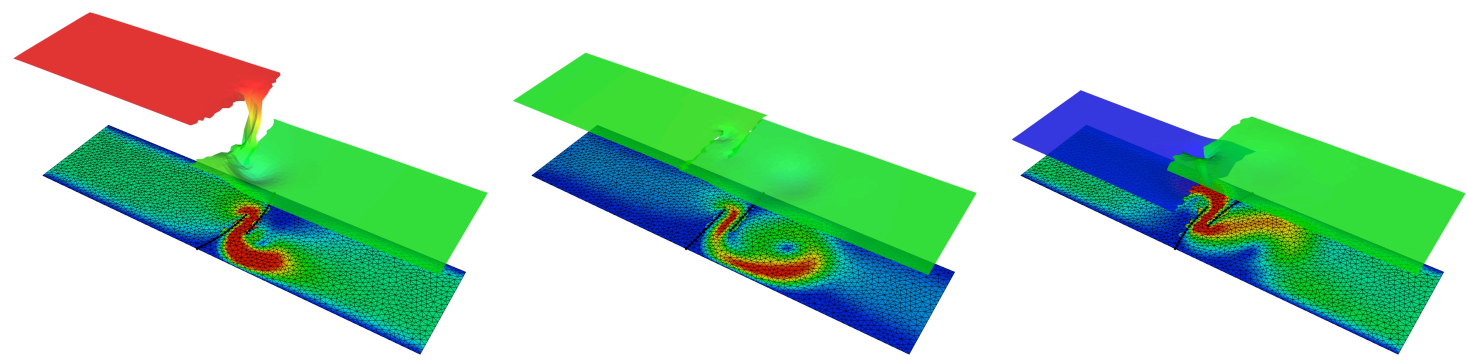

(b) Algorithm 4
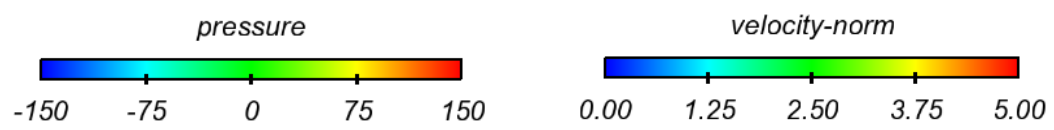

Figure 29: Snapshots of the fluid velocity magnitude and elevated pressure at time $t=0.25,0.5$ and 0.75 (from left to right) obtained with $P_{\max }=150, \tau=2.5 \cdot 10^{-3}$ and $h \approx 0.05$.

components at time $t=0.25$. We can also remark how the unfitted approximation is able to reproduce accurately strong and weak discontinuities in the case of partially intersected fluid domains (see Section 2.1.3).

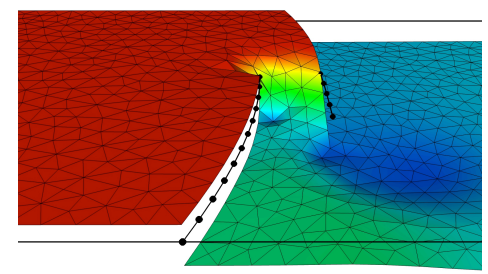

(a) Pressure

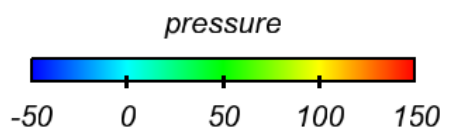

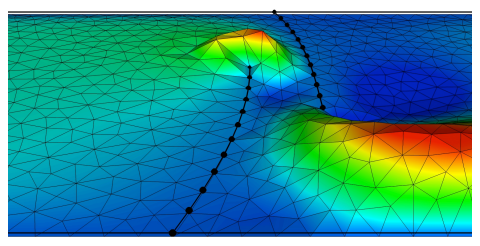

(b) Horizontal velocity

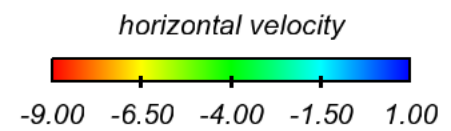

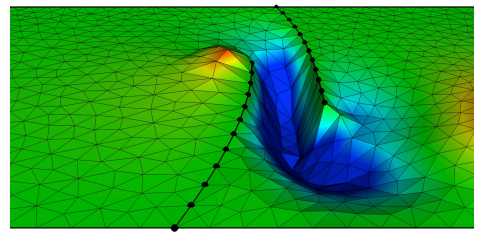

(c) Vertical velocity

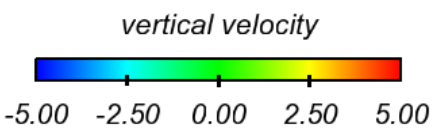

Figure 30: ALE-fitted method. Strong (pressure) and weak (velocity) discontinuities of the approximated solution at time $t=0.25$ obtained with $P_{\max }=150, \tau=2.5 \cdot 10^{-3}$ and $h \approx 0.05$.

We now turn our attention to the accuracy of the different coupling schemes proposed in 

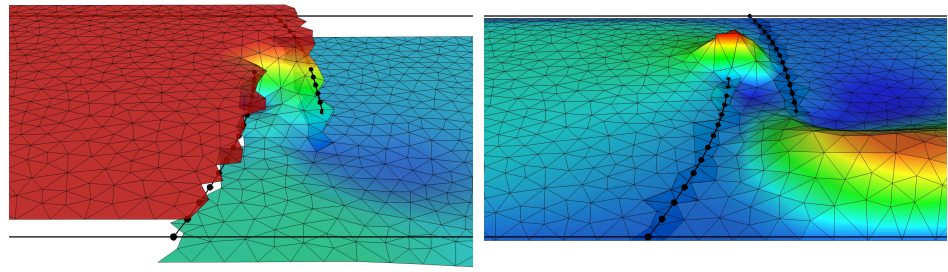

(a) Pressure

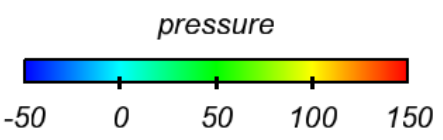

(b) Horizontal velocity

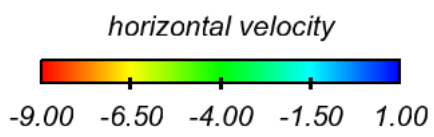

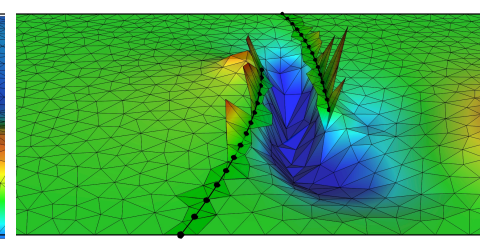

(c) Vertical velocity

vertical velocity

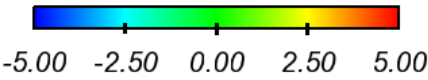

Figure 31: Algorithm 4. Strong (pressure) and weak (velocity) discontinuities of the approximated solution fields at time $t=0.25$ obtained with $P_{\max }=150, \tau=2.5 \cdot 10^{-3}$ and $h \approx 0.05$.

Section 3.2. To this purpose, we have reported in Figure 32 the time history of the solid displacement at point $(1.9,0)$ (tip of the left leaflet) obtained with Algorithms 4.6 and the implicit ALE-fitted method. Figure 32(a) confirms the results of the above qualitative discussion on the accuracy of Algorithm 4 with respect to the reference ALE-fitted method. As regards the time splitting schemes given by Algorithms 5 and Algorithm 6 . Figure 32 indicates that the best performance is obtained with the variants $(K=1, r=2)$ and $r=1,2$, respectively. Once again, this numerical evidence is consistent with the theoretical discussion of Section 2.2 ,

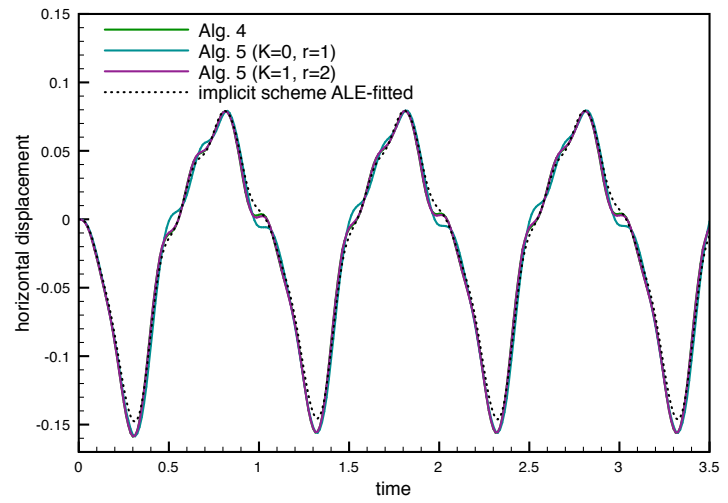

(a) Algorithms 4 and 5

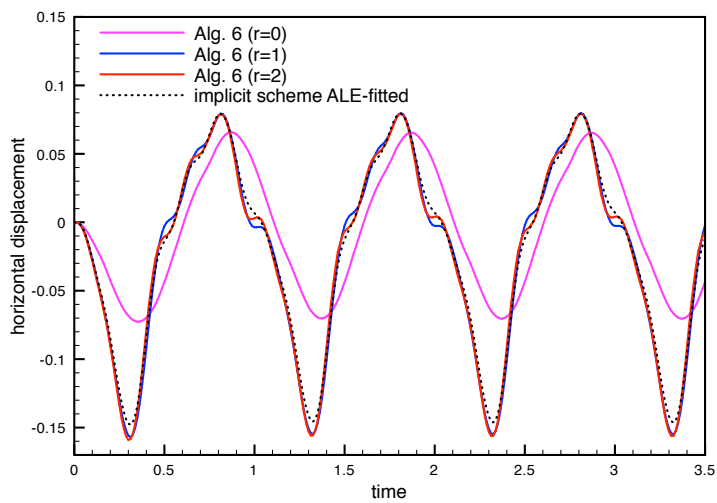

(b) Algorithm 6

Figure 32: Time history of the solid displacement at point $(1.9,0.6)$ obtained with $P_{\max }=150$, $\tau=2.5 \cdot 10^{-3}$ and $h \approx 0.05$.

Finally, in order to illustrate the capability of the proposed unfitted approach to handle very large interface deflections we have performed the simulation with an increased maximum pressure drop of $P_{\max }=400$. Since the present computer implementation is not able to handle contact, the top leaflet has been removed. Some snapshots of the discrete solution obtained with Algorithm 4 are displayed in Figure 33. Figure 34 shows the time history of the tip displacement provided by Algorithm 4 and the ALE-fitted algorithm. Both approaches give similar results up to a certain degree of interface deformation, above which the ALE-fitted method fails while the 
unfitted method still delivers a stable numerical approximation.

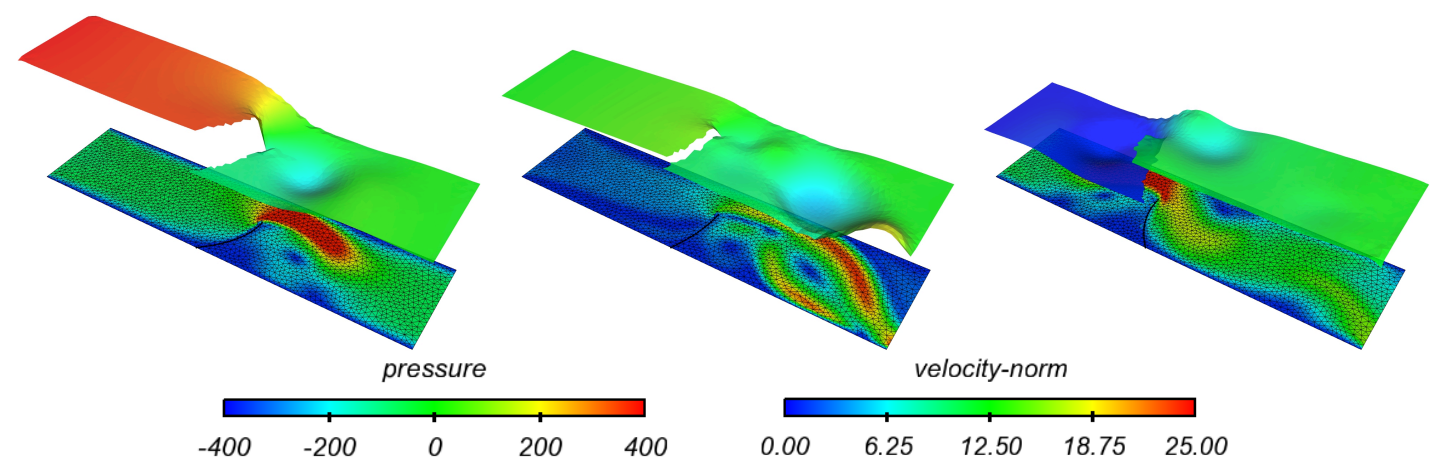

Figure 33: Snapshots of the fluid velocity magnitude and elevated pressure at time $t=0.25,0.5$ and 0.75 (from left to right) obtained with Algorithm $4 . P_{\max }=400, \tau=2.5 \cdot 10^{-3}$ and $h \approx 0.05$.

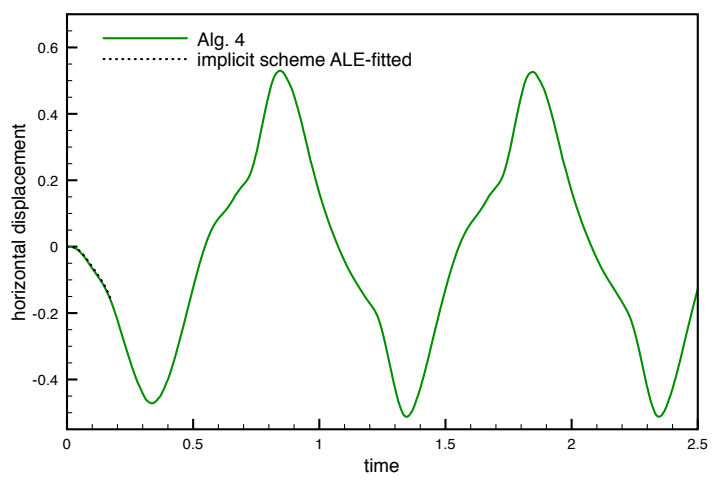

Figure 34: Time history of the solid displacement at point $(1.9,0.6)$ obtained with $P_{\max }=400$, $\tau=2.5 \cdot 10^{-3}$ and $h \approx 0.05$.

\section{Conclusion}

We have introduced a Nitsche-XFEM method for incompressible fluid-structure interaction problems involving immersed thin-walled structures. Eulerian and Lagrangian formalisms are, respectively, considered for the fluid and the solid. The key features of the spatial discretization proposed are:

- unfitted (unstructured) fluid and solid meshes;

- affine finite element approximations including weak and strong discontinuities for the velocity and the pressure, respectively (based on the XFEM method of [42, 6]);

- integration of the fluid equations only in the physical domain (cut-elements);

- consistent treatment of the kinematic/dynamic fluid-solid coupling via Nitsche's method; 
- symmetric velocity/pressure and ghost-penalty stabilization to guarantee robustness without compromising accuracy.

In the case of static interfaces, a priori error estimates (Theorem 2.1) guaranteeing optimal convergence (in the energy norm) towards non-singular solutions have been derived using the arguments reported in [18].

Several splitting schemes (implicit, explicit, semi-implicit) have been proposed, including their formulation with moving interfaces. The fundamental ingredients for the stability and accuracy of the resulting fully discrete methods have been discussed. These theoretical stability and convergence results have then been confirmed via numerical evidence in a series of $2 \mathrm{D}$ examples involving static and moving interfaces. The comparison of the different methods indicates that the best performance (in terms of accuracy and computational complexity) is obtained with Algorithms 3 and 6 with $r=1$. The salient features of this semi-implicit method are: (i) it avoids strong coupling; (ii) it simultaneously yields unconditional stability and optimal convergence in the energy norm.

Extensions of this work can explore several directions. From the modelling point of view, we plan to incorporate more complex thin-walled solid models, including contact and fracture mechanics. The extension of all these problems to 3D presents some challenges (see, e.g., the discussion of Section 2.1.4). Another interesting point, not addressed in this paper, is the convergence analysis of the methods with curved and dynamic interfaces. The latter is a particularly delicate problem which has received little consideration in the literature (see, e.g., [76] for an analysis in the parabolic case).

\section{Acknowledgements}

Work funded by the French National Research Agency (ANR) through the EXIFSI project (ANR12-JS01-0004). The authors are grateful to Prof. Erik Burman (University College London, UK) for all the fruitful discussions. They also thank an anonymous reviewer of this paper for his/her insightful remarks which helped to improve the manuscript.

\section{References}

[1] F. Alauzet. A changing-topology moving mesh technique for large displacements. Eng. Comput., 30(2):175-200, 2014.

[2] F. Alauzet and M. Mehrenberger. P1-conservative solution interpolation on unstructured triangular meshes. Internat. J. Numer. Methods Engrg., 84(13):1552-1588, 2010.

[3] R. Araya, A.H. Poza, and F. Valentin. A low-order local projection method for the incompressible Navier-Stokes equations in two- and three-dimensions. IMA J. Numer. Anal. , 2015. DOI: 10.1093/imanum/drv004.

[4] M. Astorino, J.-F. Gerbeau, O. Pantz, and K.-F. Traoré. Fluid-structure interaction and multi-body contact: application to aortic valves. Comput. Methods Appl. Mech. Engrg., 198(45-46):3603-3612, 2009.

[5] F. Baaijens. A fictitious domain/mortar element method for fluid-structure interaction. Int. Jour. Num. Meth. Fluids, 35:743-761, 2001. 
[6] R. Becker, E. Burman, and P. Hansbo. A Nitsche extended finite element method for incompressible elasticity with discontinuous modulus of elasticity. Comput. Methods Appl. Mech. Engrg., 198(41-44):3352-3360, 2009.

[7] D. Boffi, N. Cavallini, and L. Gastaldi. Finite element approach to immersed boundary method with different fluid and solid densities. Math. Models Methods Appl. Sci., 21(12):2523-2550, 2011.

[8] D. Boffi, N. Cavallini, and L. Gastaldi. The finite element immersed boundary method with distributed Lagrange multiplier. SIAM J. Numer. Anal., 53(6):2584-2604, 2015.

[9] M. Braack, E. Burman, V. John, and G. Lube. Stabilized finite element methods for the generalized Oseen problem. Comput. Methods Appl. Mech. Engrg., 196(4-6):853-866, 2007.

[10] F. Brezzi and J. Pitkäranta. On the stabilization of finite element approximations of the Stokes equations. In Efficient solutions of elliptic systems (Kiel, 1984), volume 10 of Notes Numer. Fluid Mech., pages 11-19. Vieweg, 1984.

[11] A.N. Brooks and T.J.R. Hughes. Streamline upwind/Petrov-Galerkin formulations for convection dominated flows with particular emphasis on the incompressible Navier-Stokes equations. Comput. Methods Appl. Mech. Engrg., 32(1-3):199-259, 1982.

[12] E. Burman. Ghost penalty. C. R. Math. Acad. Sci. Paris, 348(21-22):1217-1220, 2010.

[13] E. Burman, S. Claus, H. Hansbo, M. Larson, and A. Massing. CutFEM: discretizing geometry and partial differential equations. Int. J. Num. Meth. Engrg. (2015), DOI: $10.1002 /$ nme. 4823

[14] E. Burman and M.A. Fernández. Continuous interior penalty finite element method for the time-dependent Navier-Stokes equations: space discretization and convergence. Numer. Math., 107(1):39-77, 2007.

[15] E. Burman and M.A. Fernández. Stabilization of explicit coupling in fluid-structure interaction involving fluid incompressibility. Comput. Methods Appl. Mech. Engrg., 198(5-8):766$784,2009$.

[16] E. Burman and M.A. Fernández. Explicit strategies for incompressible fluid-structure interaction problems: Nitsche type mortaring versus Robin-Robin coupling. Int. J. Num. Meth. Engrg., 97(10):739-758, 2014.

[17] E. Burman and M.A. Fernández. Explicit strategies for incompressible fluid-structure interaction problems: Nitsche type mortaring versus Robin-Robin coupling. Int. J. Num. Meth. Engrg., 97(10):739-758, 2014.

[18] E. Burman and M.A. Fernández. An unfitted Nitsche method for incompressible fluidstructure interaction using overlapping meshes. Comput. Methods Appl. Mech. Engrg., 279:497-514, 2014.

[19] E. Burman, M.A. Fernández, and P. Hansbo. Continuous interior penalty finite element method for Oseen's equations. SIAM J. Numer. Anal., 44(3):1248-1274, 2006.

[20] E. Burman and P. Hansbo. Fictitious domain finite element methods using cut elements: II. A stabilized Nitsche method. Appl. Numer. Math., 62(4):328-341, 2012. 
[21] E. Burman and P. Hansbo. Fictitious domain methods using cut elements: III. A stabilized Nitsche method for Stokes' problem. ESAIM Math. Model. Numer. Anal., 48(3):859-874, 2014.

[22] D. Chapelle and K.J. Bathe. The Finite Element Analysis of Shells - Fundamentals. Springer, 2011.

[23] R. Codina. Stabilized finite element approximation of transient incompressible flows using orthogonal subscales. Comput. Methods Appl. Mech. Engrg., 191(39-40):4295-4321, 2002.

[24] G.-H. Cottet, E. Maitre, and T. Milcent. Eulerian formulation and level set models for incompressible fluid-structure interaction. M2AN Math. Model. Numer. Anal., 42(3):471492, 2008.

[25] D.A. Di Pietro and A. Ern. Mathematical aspects of discontinuous Galerkin methods, volume 69 of Mathematics $\& 3$ Applications. Springer, Heidelberg, 2012.

[26] A. Dimitrov. On singularities in the solution of three-dimensional Stokes flow and incompressible elasticity problems with corners. Internat. J. Numer. Methods Engrg., 60(4):773801, 2004.

[27] N. Diniz dos Santos, J.-F. Gerbeau, and J.-F. Bourgat. A partitioned fluid-structure algorithm for elastic thin valves with contact. Comput. Methods Appl. Mech. Engrg., 197(1920):1750-1761, 2008.

[28] A. Embar, J. Dolbow, and I. Harari. Imposing Dirichlet boundary conditions with Nitsche's method and spline-based finite elements. Internat. J. Numer. Methods Engrg., 83(7):877$898,2010$.

[29] R.P. Fedkiw. Coupling an Eulerian fluid calculation to a Lagrangian solid calculation with the ghost fluid method. J. Comput. Phys., 175(1):200-224, 2002.

[30] M.A. Fernández. Incremental displacement-correction schemes for incompressible fluidstructure interaction: stability and convergence analysis. Numer. Math., 123(1):21-65, 2013.

[31] M.A. Fernández and M. Landajuela. Splitting schemes for incompressible fluid/thin-walled structure interaction with unfitted meshes. C. R. Math. Acad. Sci. Paris, 2015., 2015. DOI: $10.1016 /$ j.crma.2015.04.003.

[32] M.A. Fernández, J. Mullaert, and M. Vidrascu. Explicit Robin-Neumann schemes for the coupling of incompressible fluids with thin-walled structures. Comput. Methods Appl. Mech. Engrg., 267:566-593, 2013.

[33] P.J. Frey and P.L. George. Mesh generation. Application to finite elements. ISTE Ltd and John Wiley \& Sons, 2nd edition, 2008.

[34] T.-P. Fries and T. Belytschko. The extended/generalized finite element method: an overview of the method and its applications. Internat. J. Numer. Methods Engrg., 84(3):253-304, 2010 .

[35] T.-P. Fries and A. Zilian. On time integration in the XFEM. Internat. J. Numer. Methods Engrg., 79(1):69-93, 2009.

[36] S.r Ganesan and L. Tobiska. Stabilization by local projection for convection-diffusion and incompressible flow problems. J. Sci. Comput., 43(3):326-342, 2010. 
[37] M. Gee, E. Ramm, and W.-A. Wal. Parallel multilevel solution of nonlinear shell structures. Comput. Methods Appl. Mech. Engrg., 194(21-24):2513-2533, 2005.

[38] P.L. George, H. Borouchaki, and E. Saltel. 'Ultimate' robustness in meshing an arbitrary polyhedron. Int. J. Numer. Meth. Engng., 58(7):1061-1089, 2003.

[39] Axel Gerstenberger and W.A. Wall. An extended finite element method/Lagrange multiplier based approach for fluid-structure interaction. Comput. Methods Appl. Mech. Engrg., 197(19-20):1699-1714, 2008.

[40] R. Glowinski, T.W. Pan, T.I. Hesla, and D.D. Joseph. A distributed Lagrange mutiplier/fictitious domain method for particulate flows. Int. J. of Multiphase Flow, 25:755-794, 1999.

[41] A. Hansbo and P. Hansbo. An unfitted finite element method, based on Nitsche's method, for elliptic interface problems. Comput. Methods Appl. Mech. Engrg., 191(47-48):5537-5552, 2002 .

[42] A. Hansbo and P. Hansbo. A finite element method for the simulation of strong and weak discontinuities in solid mechanics. Comput. Methods Appl. Mech. Engrg., 193(33-35):35233540, 2004.

[43] P. Hansbo. Nitsche's method for interface problems in computational mechanics. GAMMMitt., 28(2):183-206, 2005.

[44] P. Hansbo, J. Hermansson, and T. Svedberg. Nitsche's method combined with space-time finite elements for ALE fluid-structure interaction problems. Comput. Methods Appl. Mech. Engrg., 193(39-41):4195-4206, 2004.

[45] I. Harari and T.J.R. Hughes. What are $C$ and $h$ ?: inequalities for the analysis and design of finite element methods. Comput. Methods Appl. Mech. Engrg., 97(2):157-192, 1992.

[46] T.J.R. Hughes, L.P. Franca, and M. Balestra. A new finite element formulation for computational fluid dynamics. V. Circumventing the Babuška-Brezzi condition: a stable PetrovGalerkin formulation of the Stokes problem accommodating equal-order interpolations. Comput. Methods Appl. Mech. Engrg., 59(1):85-99, 1986.

[47] D. Kamensky, M.-C. Hsu, D. Schillinger, J.A. Evans, A. Aggarwal, Y. Bazilevs, M.S. Sacks, and T.J.R. Hughes. An immersogeometric variational framework for fluid-structure interaction: Application to bioprosthetic heart valves. Comput. Methods Appl. Mech. Engrg., 284:1005-1053, 2015.

[48] A. Legay, J. Chessa, and T. Belytschko. An Eulerian-Lagrangian method for fluid-structure interaction based on level sets. Comput. Methods Appl. Mech. Engrg., 195(17-18):2070-2087, 2006.

[49] C. Lehrenfeld and A. Reusken. Nitsche-XFEM with streamline diffusion stabilization for a two-phase mass transport problem. SIAM J. Sci. Comput., 34(5):A2740-A2759, 2012.

[50] C. Lehrenfeld and A. Reusken. Analysis of a Nitsche XFEM-DG discretization for a class of two-phase mass transport problems. SIAM J. Numer. Anal., 51(2):958-983, 2013.

$\mathrm{RR} \mathrm{n}^{\circ} 8723$ 
[51] M. Lombardi, N. Parolini, A. Quarteroni, and G. Rozza. Numerical simulation of sailing boats: Dynamics, FSI, and shape optimization. In G. Buttazzo and A. Frediani, editors, Variational Analysis and Aerospace Engineering: Mathematical Challenges for Aerospace Design, Springer Optimization and Its Applications, pages 339-377. Springer, 2012.

[52] A. Massing, M.G. Larson, A. Logg, and M.E. Rognes. A stabilized Nitsche fictitious domain method for the Stokes problem. J. Sci. Comput., 61(3):604-628, 2014.

[53] André Massing, Mats G. Larson, and Anders Logg. Efficient implementation of finite element methods on nonmatching and overlapping meshes in three dimensions. SIAM J. Sci. Comput., 35(1):C23-C47, 2013.

[54] U.M. Mayer, A. Gerstenberger, and W.A. Wall. Interface handling for three-dimensional higher-order XFEM-computations in fluid-structure interaction. Internat. J. Numer. Methods Engrg., 79(7):846-869, 2009.

[55] U.K. Müller, A. Wasim, E. Fontaine, O. Berg, Y. Cao, D. Lentink, S. Kranenbarg, and J.L. van Leeuwen. Fish and Flag - Exploring Fluid-Structure Interaction during Undulatory Swimming in Fish. In C.T. Lim and J.C.H. Goh, editors, 6th World Congress of Biomechanics (WCB 2010). August 1-6, 2010 Singapore, volume 31 of IFMBE Proceedings, pages 44-47. Springer, 2010.

[56] E.P. Newren, A.L. Fogelson, R.D. Guy, and R.M. Kirby. Unconditionally stable discretizations of the immersed boundary equations. J. Comput. Phys., 222(2):702-719, 2007.

[57] J. Nitsche. Über ein Variationsprinzip zur Lösung von Dirichlet-Problemen bei Verwendung von Teilräumen, die keinen Randbedingungen unterworfen sind. Abh. Math. Sem. Univ. Hamburg, 36:9-15, 1971.

[58] C.S. Peskin. The immersed boundary method. Acta Numer., 11:479-517, 2002.

[59] C. Pozrikidis. Computational hydrodynamics of capsules and biological cells. Chapman \& Hall/CRC Mathematical and Computational Biology. CRC Press, 2010.

[60] T. Richter. A fully Eulerian formulation for fluid-structure-interaction problems. J. Comput. Phys., 233:227-240, 2013.

[61] M. Ruess, D. Schillinger, A.I. Özcan, and E. Rank. Weak coupling for isogeometric analysis of non-matching and trimmed multi-patch geometries. Comput. Methods Appl. Mech. Engrg., 269:46-71, 2014.

[62] T. Sawada and A. Tezuka. LLM and X-FEM based interface modeling of fluid-thin structure interactions on a non-interface-fitted mesh. Comput. Mech., 48(3):319-332, 2011.

[63] B. Schott and W.A. Wall. A new face-oriented stabilized XFEM approach for 2D and 3D incompressible Navier-Stokes equations. Comput. Methods Appl. Mech. Engrg., 276:233-265, 2014.

[64] K. Stein, T.E. Tezduyar, and R. Benney. Automatic mesh update with the solid-extension mesh moving technique. Comput. Methods Appl. Mech. Engrg., 193(21-22):2019-2032, 2004.

[65] R. Stenberg. On some techniques for approximating boundary conditions in the finite element method. J. Comput. Appl. Math., 63(1-3):139-148, 1995. 
[66] Y. Sudhakar, J.P. Moitinho de Almeida, and W.A. Wall. An accurate, robust, and easyto-implement method for integration over arbitrary polyhedra: Application to embedded interface methods. J. Comput. Phys., 273:393-415, 2014.

[67] K. Takizawa and T.E. Tezduyar. Computational methods for parachute fluid-structure interactions. Arch. Comput. Methods Eng., 19:125-169, 2012.

[68] K. Takizawa, T.E. Tezduyar, A. Buscher, and S. Asada. Space-time interface-tracking with topology change (ST-TC). Comput. Mech., 54(4):955-971, 2014.

[69] T.E. Tezduyar. Stabilized finite element formulations for incompressible flow computations. In Advances in applied mechanics, Vol. 28, volume 28 of Adv. Appl. Mech., pages 1-44. Academic Press, Boston, MA, 1992.

[70] F.-B. Tian, H. Dai, H. Luo, J.F. Doyle, and B. Rousseau. Fluid-structure interaction involving large deformations: 3D simulations and applications to biological systems. J. Comput. Phys., 258:451-469, 2014.

[71] K. Wang, J. Grétarsson, A. Main, and C. Farhat. Computational algorithms for tracking dynamic fluid-structure interfaces in embedded boundary methods. Internat. J. Numer. Methods Fluids, 70(4):515-535, 2012.

[72] T. Wick. Fluid-structure interactions using different mesh motion techniques. Comp. 86 Struct., 89(13-14):1456-1467, 2011.

[73] S. Yigit, M. Schäfer, and M. Heck. Grid movement techniques and their influence on laminar fluid-structure interaction computations. J. Fluids Struct., 24(6):819-832, 2008.

[74] L. Zhang, A. Gerstenberger, X. Wang, and W.K. Liu. Immersed finite element method. Comput. Methods Appl. Mech. Engrg., 193(21-22):2051-2067, 2004.

[75] A. Zilian and A. Legay. The enriched space-time finite element method (EST) for simultaneous solution of fluid-structure interaction. Internat. J. Numer. Methods Engrg., 75(3):305$334,2008$.

[76] P. Zunino. Analysis of backward Euler/extended finite element discretization of parabolic problems with moving interfaces. Comput. Methods Appl. Mech. Engrg., 258:152-165, 2013. 


\section{Contents}

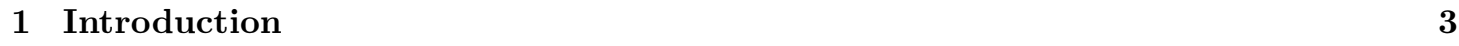

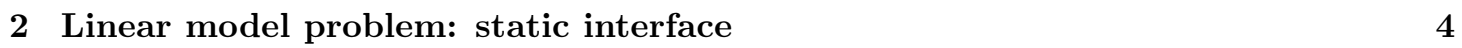

2.1 Space semi-discretization . . . . . . . . . . . . . . . . . . . 6

2.1 .1 Nitsche-XFEM semi-discrete formulation . . . . . . . . . . . . . . . . 6

$2.1 .2 \quad$ Stability and convergence . . . . . . . . . . . . . . . . . . . . . . . . . . . . . .

2.1.3 Partially intersected fluid domain . . . . . . . . . . . . . . . . . . . 11

2.1 .4 Mesh intersection and integrals over cut-elements . . . . . . . . . . . . . . 13

2.2 Time discretization: fully discrete schemes . . . . . . . . . . . . . . . . . . . 14

2.2 .1 Implicit coupling scheme . . . . . . . . . . . . . . . . . . . . . . . . . . . . . . . . . . . . 15

$2.2 .2 \quad$ Stabilized explicit schemes . . . . . . . . . . . . . . . . . . . . . . . . . . . . . . . . . . . 16

2.2 .3 Semi-implicit schemes . . . . . . . . . . . . . . . . . . . 18

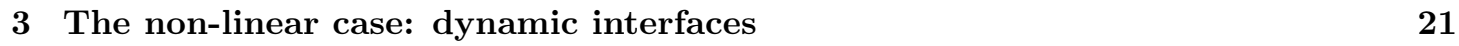

3.1 Problem setting . . . . . . . . . . . . . . . . . . . . . . . . 21

3.2 Numerical methods . . . . . . . . . . . . . . . . . . . . . . . . . . . . . . . . . . . 22

$3.2 .1 \quad$ Nitsche-XFEM formulation and coupling schemes . . . . . . . . . . . . . 23

3.2.2 Integrals with discontinuous functions at different time levels . . . . . . . 26

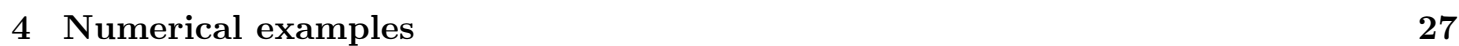

4.1 Static interface . . . . . . . . . . . . . . . . . . . . . . . . . . . . . 28

$4.1 .1 \quad$ Idealized closed valve . . . . . . . . . . . . . . . . . . . 28

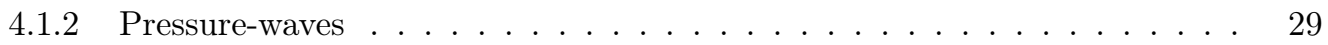

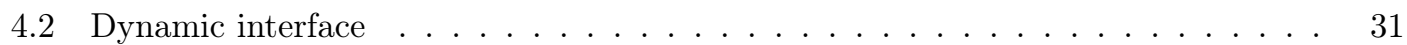

4.2 .1 Idealized closed valve . . . . . . . . . . . . . . . . . . . . . . . . . . . . . . . . . . . . . . . .

4.2 .2 Double cavity . . . . . . . . . . . . . . . . . . . . . . . . . . . . . . . . . . . . 34

$4.2 .3 \quad$ Idealized open valve . . . . . . . . . . . . . . . . . . . . . 38

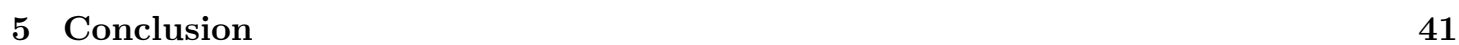




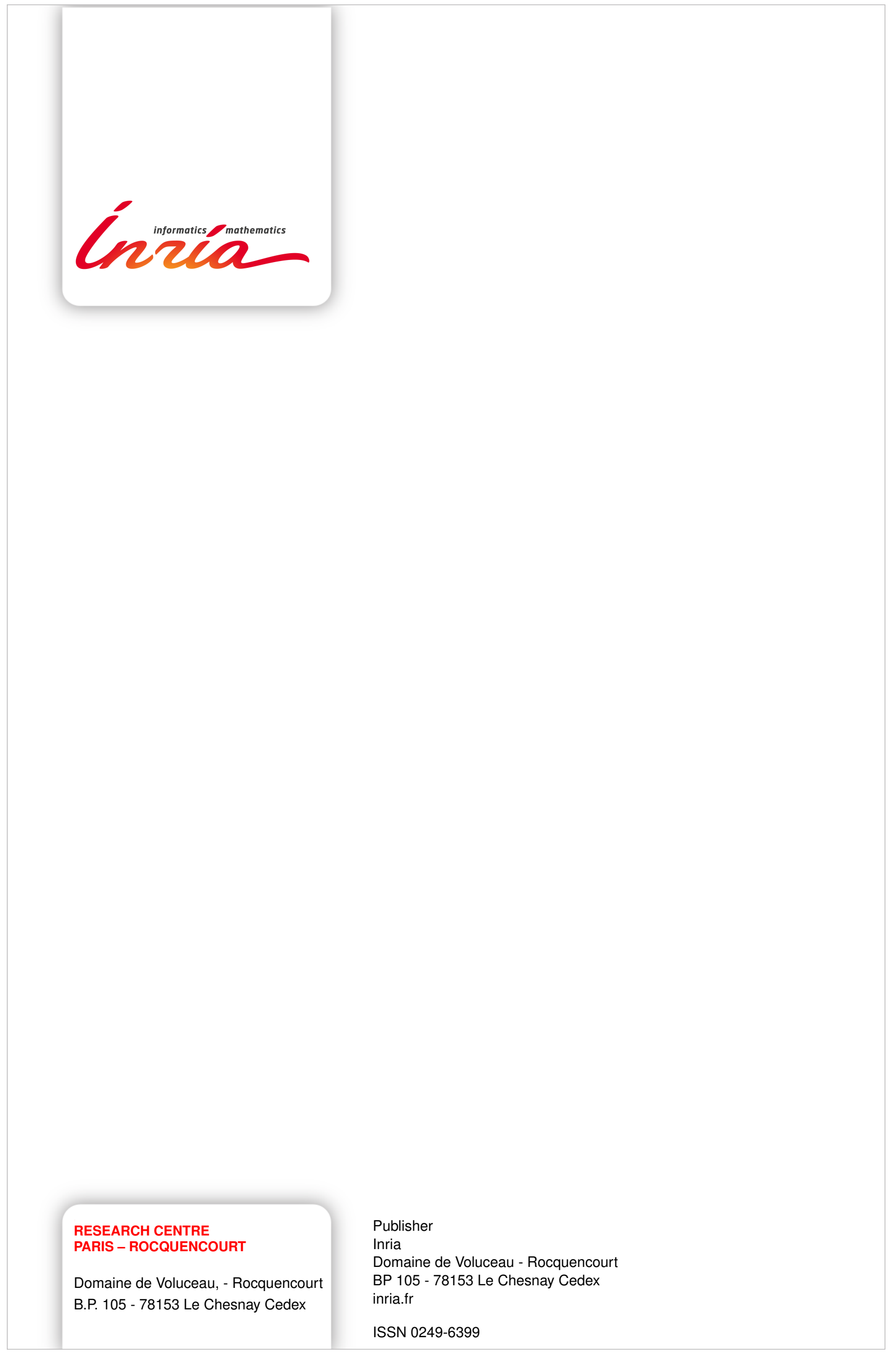

